\title{
Physical conditions of the molecular gas in metal-poor galaxies ${ }^{\star}$
}

\author{
L. K. Hunt ${ }^{1}$, A. Weiß ${ }^{2}$, C. Henkel $^{2,3}$, F. Combes ${ }^{4}$, S. García-Burillo ${ }^{5}$, V. Casasola ${ }^{1}$, P. Caselli ${ }^{6}$, A. Lundgren ${ }^{9}$, \\ R. Maiolino ${ }^{7}$, K. M. Menten ${ }^{2}$, and L. Testi ${ }^{1,8}$ \\ 1 INAF-Osservatorio Astrofisico di Arcetri, Largo E. Fermi, 5, 50125 Firenze, Italy \\ e-mail: hunt@arcetri.astro.it \\ 2 Max-Planck-Institut für Radioastronomie, Auf dem Hügel 69, 53121 Bonn, Germany \\ 3 Astronomy Department, King Abdulaziz University, PO Box 80203, Jeddah, Saudia Arabia \\ ${ }^{4}$ Observatoire de Paris, LERMA, Collège de France, CNRS, PSL, Sorbonne University UPMC, 75014 Paris, France \\ 5 Observatorio Astronómico Nacional (OAN)-Observatorio de Madrid, Alfonso XII, 3, 28014 Madrid, Spain \\ ${ }^{6}$ Max-Planck-Institut für extraterrestrische Physik, Giessenbachstrasse 1, 85748 Garching, Germany \\ 7 Cavendish Laboratory, University of Cambridge, 19 J.J. Thomson Avenue, Cambridge CB3 0HE, UK \\ 8 ESO, Karl Schwarzschild str. 2, 85748 Garching bei München, Germany \\ 9 Vittja 64, 74793 Alunda, Sweden
}

Received 18 April 2017 / Accepted 8 August 2017

\begin{abstract}
Studying the molecular component of the interstellar medium (ISM) in metal-poor galaxies has been challenging because of the faintness of carbon monoxide emission, the most common proxy of $\mathrm{H}_{2}$. Here we present new detections of molecular gas at low metallicities, and assess the physical conditions in the gas through various CO transitions for 8 galaxies. For one, NGC $1140\left(Z / Z_{\odot} \sim 0.3\right)$, two detections of ${ }^{13} \mathrm{CO}$ isotopologues and atomic carbon, $[\mathrm{CI}](1-0)$ and an upper limit for $\mathrm{HCN}(1-0)$ are also reported. After correcting to a common beam size, we compared ${ }^{12} \mathrm{CO}(2-1) /{ }^{12} \mathrm{CO}(1-0)\left(R_{21}\right)$ and ${ }^{12} \mathrm{CO}(3-2) /{ }^{12} \mathrm{CO}(1-0)\left(R_{31}\right)$ line ratios of our sample with galaxies from the literature and find that only NGC 1140 shows extreme values $\left(R_{21} \sim R_{31} \sim 2\right)$. Fitting physical models to the ${ }^{12} \mathrm{CO}$ and ${ }^{13} \mathrm{CO}$ emission in NGC 1140 suggests that the molecular gas is cool (kinetic temperature $\left.T_{\text {kin }} \lesssim 20 \mathrm{~K}\right)$ ), dense $\left(\mathrm{H}_{2}\right.$ volume density $\left.n_{\mathrm{H} 2} \gtrsim 10^{6} \mathrm{~cm}^{-3}\right)$, with moderate $\mathrm{CO}$ column density $\left(N_{\mathrm{CO}} \sim 10^{16} \mathrm{~cm}^{-2}\right)$ and low filling factor. Surprisingly, the $\left[{ }^{12} \mathrm{CO}\right] /\left[{ }^{13} \mathrm{CO}\right]$ abundance ratio in NGC 1140 is very low $(\sim 8-20)$, lower even than the value of 24 found in the Galactic Center. The young age of the starburst in NGC 1140 precludes ${ }^{13} \mathrm{CO}$ enrichment from evolved intermediate-mass stars; instead we attribute the low ratio to charge-exchange reactions and fractionation, because of the enhanced efficiency of these processes in cool gas at moderate column densities. Fitting physical models to ${ }^{12} \mathrm{CO}$ and $[\mathrm{CI}](1-0)$ emission in NGC 1140 gives an unusually low $\left[{ }^{12} \mathrm{CO}\right] /\left[{ }^{12} \mathrm{C}\right]$ abundance ratio, suggesting that in this galaxy atomic carbon is at least 10 times more abundant than ${ }^{12} \mathrm{CO}$.
\end{abstract}

Key words. galaxies: starburst - galaxies: dwarf - galaxies: star formation - galaxies: ISM - ISM: molecules - radio lines: ISM

\section{Introduction}

Physical conditions in the molecular component of the interstellar medium (ISM) of metal-poor galaxies are difficult to measure. The main obstacle is the faintness at low metallicities of the common proxy of $\mathrm{H}_{2}$, carbon monoxide emission (Sage et al. 1992; Taylor et al. 1998; Gondhalekar et al. 1998; Barone et al. 2000; Leroy et al. 2005, 2007; Buyle et al. 2006; Schruba et al. 2012; Cormier et al. 2014). While the proximity of Local Group galaxies facilitates studies of molecular gas (e.g., Cohen et al. 1988; Rubio et al. 1993, 2015; Fukui et al. 1999; Israel et al. 2003; Bolatto et al. 2008; Pineda et al. 2012; Elmegreen et al. 2013; Paron et al. 2014; Shi et al. 2015; Paron et al. 2016; Shi et al. 2016; Schruba et al. 2017), beyond the Local Group, CO detections in low-metallicity galaxies are arduous (e.g., Schruba et al. 2012; Cormier et al. 2014), and few in number.

* Based on observations carried out with the IRAM $30 \mathrm{~m}$ and the Atacama Pathfinder Experiment (APEX). IRAM is supported by the INSU/CNRS (France), MPG (Germany), and IGN (Spain), and APEX is a collaboration between the Max-Planck-Institut fur Radioastronomie, the European Southern Observatory, and the Onsala Space Observatory.
In a previous paper (Hunt et al. 2015, hereafter Paper I), we reported ${ }^{12} \mathrm{CO}(1-0)$ detections in 8 metal-poor galaxies outside the Local Group with metallicities ranging from $12+\log (\mathrm{O} / \mathrm{H}) \sim 7.7$ to 8.4 , or equivalently $0.1 Z_{\odot}$ to $0.5 Z_{\odot}{ }^{1}$. This more than doubles the previous number of such detections, and sets the stage for a more detailed study of the physical conditions in molecular gas in a metal-poor ISM.

In this paper, we present observations of ${ }^{12} \mathrm{CO}(2-1)$ and ${ }^{12} \mathrm{CO}(3-2)$ for some of the galaxies in Paper I, and for one of them, NGC 1140, also ${ }^{12} \mathrm{CO}(4-3),{ }^{13} \mathrm{CO}(1-0),{ }^{13} \mathrm{CO}(2-1)$, and $[\mathrm{CI}](1-0)$ detections, as well as upper limits for other molecular transitions including $\mathrm{HCN}(1-0)$. In Sect. 2, we briefly discuss the sample, and in Sect. 3, describe the observations and data reduction. Section 4 presents our approach for correcting the observed temperatures and fluxes for the different beam sizes. Section 5 analyzes the observed line ratios from an empirical point of view, comparing them with other metal-rich samples, and Sect. 6 discusses dense-gas tracers. Physical models of the line emission of NGC 1140 are presented in Sect. 7, in order to infer $\mathrm{H}_{2}$ volume

\footnotetext{
1 We assume the Asplund et al. (2009) solar abundance calibration of $12+\log (\mathrm{O} / \mathrm{H})=8.69$.
} 
Table 1. Parameters for observed galaxies.

\begin{tabular}{|c|c|c|c|c|c|c|c|c|c|}
\hline \multirow[t]{2}{*}{ Name } & \multicolumn{2}{|c|}{ Pointed position (J2000) } & \multirow[t]{2}{*}{ Redshift } & \multirow{2}{*}{$\begin{array}{c}\text { Distance } \\
(\mathrm{Mpc})\end{array}$} & \multirow{2}{*}{$\begin{array}{l}\text { Distance } \\
\text { method }^{a}\end{array}$} & \multirow[t]{2}{*}{$\operatorname{Size}^{b}$} & \multicolumn{3}{|c|}{ Beam $F W H M$} \\
\hline & RA & Dec & & & & & ${ }^{12} \mathrm{CO}(1-0)$ & ${ }^{12} \mathrm{CO}(2-1)$ & ${ }^{12} \mathrm{CO}(3-2)$ \\
\hline \multirow{2}{*}{ CGCG 007-025 } & 09:44:01.9 & $-00: 38: 32.0$ & 0.00483 & 24.5 & $\mathrm{CMB}$ & $27^{\prime \prime} \times 16^{\prime \prime} \cdot 2$ & $21 " 44$ & $10 ! 7$ & - \\
\hline & & & & & & & $2.54 \mathrm{kpc}$ & $1.27 \mathrm{kpc}$ & - \\
\hline \multirow{2}{*}{ II Zw 40} & $05: 55: 42.6$ & $03: 23: 31.5$ & 0.00263 & 11.7 & CMB & $33 " .6 \times 13 ": 2$ & - & $27 " .1$ & $18 " .1$ \\
\hline & & & & & & & - & $1.537 \mathrm{kpc}$ & $1.027 \mathrm{kpc}$ \\
\hline \multirow{2}{*}{ Mrk 996} & $01: 27: 35.5$ & $-06: 19: 36.0$ & 0.00541 & 18.1 & CMB & $36^{\prime \prime} \times 30^{\prime \prime}$ & $21 " .5$ & $10 \prime: 7^{\prime}$ & $18 " .1$ \\
\hline & & & & & & & $1.88 \mathrm{kpc}$ & $0.94 \mathrm{kpc}$ & $1.59 \mathrm{kpc}$ \\
\hline \multirow[t]{2}{*}{ NGC 1140} & 02:54:33.6 & $-10: 01: 40.0$ & 0.00501 & 19.7 & $\mathrm{TF}$ & $102^{\prime \prime} \times 54^{\prime \prime}$ & $21 " .4$ & $10^{\prime \prime} \cdot 7$ & $18{ }^{\prime \prime} 1$ \\
\hline & & & & & & & $2.04 \mathrm{kpc}$ & $1.02 \mathrm{kpc}$ & $1.73 \mathrm{kpc}$ \\
\hline \multirow[t]{2}{*}{ NGC 1156} & $02: 59: 42.2$ & 25:14:14.0 & 0.00125 & 8.1 & Stars & $198^{\prime \prime} \times 150^{\prime \prime}$ & $21^{\prime \prime} 4$ & $10^{\prime \prime} 7$ & - \\
\hline & & & & & & & $0.84 \mathrm{kpc}$ & $0.42 \mathrm{kpc}$ & \\
\hline \multirow[t]{2}{*}{ NGC 3353 (Haro 3) } & $10: 45: 22.40$ & $55: 57: 37.0$ & 0.00315 & 18.1 & $\mathrm{TF}$ & $72^{\prime \prime} \times 49^{\prime \prime} .8$ & $21 ": 4$ & $10 !: 7$ & - \\
\hline & & & & & & & $1.88 \mathrm{kpc}$ & $0.94 \mathrm{kpc}$ & \\
\hline \multirow[t]{2}{*}{ NGC 7077} & 21:29:59.6 & $02: 24: 51.0$ & 0.00384 & 17.2 & $\mathrm{TF}$ & $48^{\prime \prime} \times 42^{\prime \prime}$ & $21 " .4$ & $10^{\prime \prime} \cdot 7$ & $18 " 1$ \\
\hline & & & & & & & $1.78 \mathrm{kpc}$ & $0.89 \mathrm{kpc}$ & $1.51 \mathrm{kpc}$ \\
\hline \multirow[t]{2}{*}{ SBS 0335-052 } & $03: 37: 44.0$ & $-05: 02: 40.0$ & 0.01352 & 53.6 & CMB & $13 ": 8 \times 12 " .0$ & - & $27 ": 4$ & $18 " 3$ \\
\hline & & & & & & & - & $7.12 \mathrm{kpc}$ & $4.76 \mathrm{kpc}$ \\
\hline \multirow[t]{2}{*}{ UM 448} & $11: 42: 12.4$ & 00:20:03.0 & 0.01856 & 81.2 & CMB & $24^{\prime \prime} \times 24^{\prime \prime}$ & $21 " 77$ & $10 \prime 99$ & $18 " .4$ \\
\hline & & & & & & & $8.54 \mathrm{kpc}$ & $4.29 \mathrm{kpc}$ & $7.244, \mathrm{kpc}$ \\
\hline \multirow[t]{2}{*}{ UM 462} & $11: 52: 37.2$ & $-02: 28: 10.0$ & 0.00353 & 19.5 & CMB & $36^{\prime \prime} \times 30^{\prime \prime}$ & $21 " .4$ & $10^{\prime \prime} 7$ & $18 " .1$ \\
\hline & & & & & & & $2.02 \mathrm{kpc}$ & $1.01 \mathrm{kpc}$ & $1.71 \mathrm{kpc}$ \\
\hline
\end{tabular}

Notes. ${ }^{(a)}$ Taken from NED: Cosmic Microwave Background (CMB), Tully Fisher (TF), Stars. ${ }^{(b)}$ Taken from NED.

density, $n_{\mathrm{H} 2}$, kinetic temperature, $T_{\text {kin }}$, $\mathrm{CO}$ column density, $N_{\mathrm{CO}}$, and the ${ }^{12} \mathrm{CO} /{ }^{13} \mathrm{CO}$ as well as the $[\mathrm{CI}] /{ }^{12} \mathrm{CO}$ abundance ratios. More limited models are computed for the other galaxies with fewer multiple transitions. We discuss the results in Sect. 8, and give our conclusions in Sect. 9.

\section{The targets}

A complete description of source selection and the individual galaxies is given in Paper I. Here we briefly outline the main selection criteria and general characteristics of our targets.

The majority of the galaxies in the observing sample already had $\mathrm{H}_{2}$ detections, either of the ro-vibrational transitions at $2 \mu \mathrm{m}$ or the rotational transitions in the mid-infrared with Spitzer/IRS (Hunt et al. 2010). Additional galaxies were included with previous $\mathrm{CO}$ observations lacking a clear detection. Stellar masses, estimated from $3.6 \mu \mathrm{m}$ luminosities after the subtraction of nebular emission, range from $\sim 10^{8}$ to $10^{10} M_{\odot}$ (UM448 is more massive than this, and is the clear result of an interaction with another galaxy, see Paper I for more details). The relatively high specific star-formation rates (ratio of SFR and stellar mass, $\left.S F R / M_{\text {star }}=s S F R\right)$ of the sample make them starburst galaxies $\left(s S F R \gtrsim 10^{-10} \mathrm{yr}^{-1}\right)$. They are also gas rich, with gas-mass fractions (relative to total baryonic mass) as high as $\sim 0.8$.

\section{The observations}

We have observed ${ }^{12} \mathrm{CO}(1-0)$ and ${ }^{12} \mathrm{CO}(2-1)$ in our sample galaxies over a three-year period from 2008 to 2010 with the IRAM 30-m telescope (Pico Veleta, Spain). The measurements of ${ }^{12} \mathrm{CO}(3-2)$ and for a few galaxies also of ${ }^{12} \mathrm{CO}(2-1)$ were acquired with the APEX 12-m telescope (Chile) ${ }^{2}$. NGC 1140 was singled out for a more intense observing campaign in which we observed ${ }^{13} \mathrm{CO}(1-0), \mathrm{HCN}(1-0)$, other 3-mm transitions, and

\footnotetext{
2 This publication is based on data acquired with the Atacama Pathfinder Experiment (APEX). APEX is a collaboration between the Max-Planck-Institut für Radioastronomie, the European Southern Observatory, and the Onsala Space Observatory.
}

${ }^{13} \mathrm{CO}(2-1)$ at the $30 \mathrm{~m} ;{ }^{12} \mathrm{CO}(4-3)$ and $[\mathrm{CI}](1-0)$ measurements were obtained with APEX. The ${ }^{12} \mathrm{CO}(1-0)$ observations of this sample were presented in Paper I. Table 1 reports selected observational parameters for the sample galaxies. Our single-dish observations sample fairly large regions within each galaxy, ranging from $420 \mathrm{pc}$ for the smallest beam in the closest galaxy $\left({ }^{12} \mathrm{CO}(2-1)\right.$, NGC 1156$)$ to $8.5 \mathrm{kpc}$ for the largest beam in the most distant one $\left({ }^{12} \mathrm{CO}(1-0)\right.$, UM 448).

\subsection{IRAM}

We observed eight galaxies at the $30 \mathrm{~m}$ in ${ }^{12} \mathrm{CO}(1-0)$ and ${ }^{12} \mathrm{CO}(2-1)$, first with the older $\mathrm{ABCD}$ receivers (proposals 03608, 227-09), then with the Eight Mixer Receiver (EMIR, proposal 097-10) using the Wideband Line Multiple Autocorrelator (WILMA) backend. Earlier spectra were acquired at an intrinsic resolution of $4 \mathrm{MHz}$, while later ones using EMIR at $2 \mathrm{MHz}$. For NGC 1140, the EMIR E090 receiver was tuned to an intermediate frequency, $\sim 113 \mathrm{GHz}$, in order to cover other $3 \mathrm{~mm}$ transitions simultaneously together with ${ }^{12} \mathrm{CO}(1-0)$ in the lower-inner sideband in two polarizations. We adopted wobbler switching with a throw of 90", and used the standard intensity calibration with two absorbers at different temperatures. Pointing was checked every hour or two on nearby planets or bright quasars, and focus tests were performed every $4 \mathrm{~h}$ during the night and every $3 \mathrm{~h}$ during the day. Typical pointing errors were $\lesssim 2^{\prime \prime}$, and never exceeded $\sim 3^{\prime \prime}$.

With the EMIR setup for NGC 1140, we were able to also observe additional transitions, including ${ }^{13} \mathrm{CO}(1-0),{ }^{13} \mathrm{CO}(2-1)$, $\mathrm{C}^{18} \mathrm{O}(1-0)$, and the dense-gas tracers $\mathrm{HCN}(1-0), \mathrm{CN}(1-0)$, and CS $(2-1)$.

\section{2. $A P E X$}

The ${ }^{12} \mathrm{CO}(3-2)$ observations of six of our targets were performed with the Swedish Heterodyne Facility Instrument (SHeFI) at the 12-m Atacama Pathfinder Experiment telescope (APEX). The observations were acquired over three observing periods from 
L. K. Hunt et al.: Physical conditions at low metallicity

Table 2. CO emission line parameters for CGCG 007-025, Mrk 996, NGC 1156, and NGC 3353.

\begin{tabular}{|c|c|c|c|c|c|c|c|c|c|c|c|c|c|}
\hline Name & $\begin{array}{c}\text { Systemic } \\
\text { velocity } \\
\left(\mathrm{km} \mathrm{s}^{-1}\right)\end{array}$ & Transition & Component & $\begin{array}{c}\text { Beam } \\
\text { size } \\
\left({ }^{\prime \prime}\right) \\
\end{array}$ & $\begin{array}{c}\text { Channel } \\
\text { width } \\
\left(\mathrm{km} \mathrm{s}^{-1}\right) \\
\end{array}$ & $\begin{array}{l}T_{\text {rms }} \\
(\mathrm{mK})\end{array}$ & $\begin{array}{l}T_{\text {peak }} \\
(\mathrm{mK})\end{array}$ & $\begin{array}{l}\text { Offset }^{a} \\
\left(\mathrm{~km} \mathrm{~s}^{-1}\right)\end{array}$ & $\begin{array}{l}F W H M \\
\left(\mathrm{~km} \mathrm{~s}^{-1}\right)\end{array}$ & $\begin{array}{c}I_{\mathrm{CO}} \\
\left(\mathrm{K} \mathrm{km} \mathrm{s}^{-1}\right)\end{array}$ & $\begin{array}{c}I_{\mathrm{CO}}^{\mathrm{cor} b} \\
\left(\mathrm{~K} \mathrm{~km} \mathrm{~s}^{-1}\right)\end{array}$ & $\begin{array}{c}S_{\mathrm{CO}}^{\mathrm{cor} b} \\
\left(\mathrm{Jy} \mathrm{km} \mathrm{s}^{-1}\right)\end{array}$ & $\begin{array}{c}\log \left(L_{\mathrm{cor}}^{\prime}\right)^{b} \\
\left(\mathrm{~K} \mathrm{~km} \mathrm{~s}^{-1} \mathrm{pc}^{2}\right)\end{array}$ \\
\hline CGCG 007-025 & 1449 & ${ }^{12} \mathrm{CO}(1-0)$ & Total & 21.4 & 15.7 & 1.3 & 2.3 & -34 & 129 & $0.314(0.092)$ & $0.314(0.092)$ & 1.60 & $6.368(0.126)$ \\
\hline CGCG 007-025 & & ${ }^{12} \mathrm{CO}(2-1)$ & Total & 10.7 & 10.5 & 3.9 & - & - & - & $<0.31$ & - & - & $<5.83$ \\
\hline CGCG 007-025 & & ${ }^{12} \mathrm{CO}(2-1)$ & Total & 10.7 & 15.7 & 3.4 & - & - & - & $<0.31$ & - & - & $<5.95$ \\
\hline Mrk 996 & 1622 & ${ }^{12} \mathrm{CO}(1-0)$ & 1 & 21.5 & 5.2 & 1.8 & 6.0 & -1 & 28 & $0.189(0.034)$ & $0.182(0.033)$ & 0.97 & $5.890(0.077)$ \\
\hline Mrk 996 & & ${ }^{12} \mathrm{CO}(1-0)$ & 2 & 21.5 & 5.2 & 1.8 & 4.7 & 39 & 10 & $0.054(0.019)$ & $0.052(0.019)$ & 0.28 & $5.347(0.154)$ \\
\hline Mrk 996 & & ${ }^{12} \mathrm{CO}(1-0)$ & Total & 21.5 & 5.2 & 1.8 & 6.0 & 0 & 40 & $0.243(0.039)$ & $0.235(0.037)$ & 1.25 & $5.999(0.069)$ \\
\hline Mrk 996 & & ${ }^{12} \mathrm{CO}(2-1)$ & Total & 10.7 & 5.2 & 2.5 & 3.8 & -12 & 32 & $0.291(0.047)$ & $0.128(0.020)$ & 2.69 & $5.729(0.070)$ \\
\hline Mrk 996 & & ${ }^{12} \mathrm{CO}(2-1)$ & 1 & 27.2 & 10.2 & 2.1 & 6.0 & -12 & 123 & $0.563(0.131)$ & $0.782(0.182)$ & 15.34 & $6.485(0.101)$ \\
\hline Mrk 996 & & ${ }^{12} \mathrm{CO}(2-1)$ & 2 & 27.2 & 10.2 & 2.1 & 4.0 & 72 & 36 & $0.113(0.076)$ & $0.156(0.106)$ & 3.07 & $5.786(0.295)$ \\
\hline Mrk 996 & & ${ }^{12} \mathrm{CO}(2-1)$ & Total & 27.2 & 10.2 & 2.1 & 6.0 & 0 & 84 & $0.675(0.151)$ & $0.938(0.210)$ & 18.40 & $6.564(0.097)$ \\
\hline Mrk 996 & & ${ }^{12} \mathrm{CO}(3-2)$ & Total & 18.1 & 13.6 & 1.7 & - & - & - & $<0.21$ & - & - & $<5.74$ \\
\hline NGC 1156 & 375 & ${ }^{12} \mathrm{CO}(1-0)$ & 1 & 21.4 & 10.4 & 7.1 & 12.2 & -78 & 71 & $0.933(0.406)$ & $0.929(0.404)$ & 5.00 & $5.904(0.189)$ \\
\hline NGC 1156 & & ${ }^{12} \mathrm{CO}(1-0)$ & 2 & 21.4 & 10.4 & 7.1 & 10.0 & 25 & 85 & $0.916(0.433)$ & $0.912(0.431)$ & 4.91 & $5.896(0.205)$ \\
\hline NGC 1156 & & ${ }^{12} \mathrm{CO}(1-0)$ & Total & 21.4 & 10.4 & 7.1 & 12.2 & 0 & 103 & $1.849(0.593)$ & $1.841(0.590)$ & 9.91 & $6.201(0.139)$ \\
\hline NGC 1156 & & ${ }^{12} \mathrm{CO}(2-1)$ & Total & 10.7 & 10.4 & 4.8 & 9.4 & -5 & 67 & $0.725(0.206)$ & $0.668(0.190)$ & 14.19 & $5.755(0.123)$ \\
\hline NGC $3353\left[0^{\prime \prime}, 0^{\prime \prime}\right]$ & 944 & ${ }^{12} \mathrm{CO}(1-0)$ & 1 & 21.4 & 5.2 & 5.5 & 27.6 & -31 & 60 & $1.824(0.177)$ & $1.771(0.172)$ & 9.50 & $6.880(0.042)$ \\
\hline $\operatorname{NGC} 3353\left[0^{\prime \prime}, 0^{\prime \prime}\right]$ & & ${ }^{12} \mathrm{CO}(1-0)$ & 2 & 21.4 & 5.2 & 5.5 & 19.7 & 21 & 16 & $0.339(0.112)$ & $0.329(0.109)$ & 1.76 & $6.149(0.144)$ \\
\hline NGC $3353\left[0^{\prime \prime}, 0^{\prime \prime}\right]$ & & ${ }^{12} \mathrm{CO}(1-0)$ & Total & 21.4 & 5.2 & 5.5 & 27.6 & 0 & 52 & $2.163(0.210)$ & $2.100(0.204)$ & 11.26 & $6.954(0.042)$ \\
\hline NGC $3353\left[0^{\prime \prime}, 0^{\prime \prime}\right]$ & & ${ }^{13} \mathrm{CO}(1-0)$ & Total & 22.4 & 10.9 & 2.0 & - & - & - & $<2.10$ & - & - & $<5.95$ \\
\hline NGC $3353\left[0^{\prime \prime}, 0^{\prime \prime}\right]$ & & ${ }^{12} \mathrm{CO}(2-1)$ & 1 & 10.7 & 5.2 & 7.9 & 31.7 & -33 & 42 & $2.611(0.232)$ & $1.430(0.127)$ & 30.27 & $6.781(0.039)$ \\
\hline $\operatorname{NGC} 3353\left[0^{\prime \prime}, 0^{\prime \prime}\right]$ & & ${ }^{12} \mathrm{CO}(2-1)$ & 2 & 10.7 & 5.2 & 7.9 & 19.5 & 15 & 22 & $0.814(0.175)$ & $0.446(0.096)$ & 9.43 & $6.275(0.093)$ \\
\hline NGC $3353\left[0^{\prime \prime}, 0^{\prime \prime}\right]$ & & ${ }^{12} \mathrm{CO}(2-1)$ & Total & 10.7 & 5.2 & 7.9 & 31.7 & 0 & 48 & $3.424(0.290)$ & $1.876(0.159)$ & 39.70 & $6.899(0.037)$ \\
\hline NGC $3353\left[0^{\prime \prime},+10^{\prime \prime}\right]$ & 944 & ${ }^{12} \mathrm{CO}(1-0)$ & 1 & 21.4 & 5.2 & 4.5 & 33.0 & -35 & 39 & $1.412(0.123)$ & $1.371(0.119)$ & 7.35 & $6.769(0.038)$ \\
\hline NGC $3353\left[0^{\prime \prime},+10^{\prime \prime}\right]$ & & ${ }^{12} \mathrm{CO}(1-0)$ & 2 & 21.4 & 5.2 & 4.5 & 11.8 & 19 & 36 & $0.468(0.123)$ & $0.454(0.119)$ & 2.43 & $6.289(0.114)$ \\
\hline NGC $3353\left[0^{\prime \prime},+10^{\prime \prime}\right]$ & & ${ }^{12} \mathrm{CO}(1-0)$ & Total & 21.4 & 5.2 & 4.5 & 33.0 & 0 & 54 & $1.880(0.174)$ & $1.825(0.168)$ & 9.79 & $6.893(0.040)$ \\
\hline $\operatorname{NGC} 3353\left[0^{\prime \prime},+10^{\prime \prime}\right]$ & & ${ }^{12} \mathrm{CO}(2-1)$ & 1 & 10.7 & 5.2 & 7.9 & 16.2 & -34 & 52 & $1.635(0.207)$ & $0.896(0.114)$ & 18.96 & $6.578(0.055)$ \\
\hline NGC $3353\left[0^{\prime \prime},+10^{\prime \prime}\right]$ & & ${ }^{12} \mathrm{CO}(2-1)$ & 2 & 10.7 & 5.2 & 7.9 & 4.3 & 36 & 12 & $0.103(0.079)$ & $0.056(0.044)$ & 1.19 & $5.377(0.336)$ \\
\hline $\operatorname{NGC} 3353\left[0^{\prime \prime},+10^{\prime \prime}\right]$ & & ${ }^{12} \mathrm{CO}(2-1)$ & Total & 10.7 & 5.2 & 7.9 & 16.2 & 0 & 70 & $1.738(0.221)$ & $0.952(0.121)$ & 20.15 & $6.605(0.055)$ \\
\hline NGC $3353\left[-6^{\prime \prime},-9^{\prime \prime}\right]$ & 944 & ${ }^{12} \mathrm{CO}(1-0)$ & 1 & 21.4 & 5.2 & 4.6 & 6.7 & -56 & 29 & $0.217(0.037)$ & $0.211(0.036)$ & 1.13 & $5.955(0.075)$ \\
\hline NGC $3353\left[-6^{\prime \prime},-9^{\prime \prime}\right]$ & & ${ }^{12} \mathrm{CO}(1-0)$ & 2 & 21.4 & 5.2 & 4.6 & 13.6 & -16 & 28 & $0.407(0.037)$ & $0.395(0.036)$ & 2.12 & $6.229(0.040)$ \\
\hline $\operatorname{NGC} 3353\left[-6^{\prime \prime},-9^{\prime \prime}\right]$ & & ${ }^{12} \mathrm{CO}(1-0)$ & 3 & 21.4 & 5.2 & 4.6 & 20.0 & 22 & 35 & $0.758(0.037)$ & $0.736(0.036)$ & 3.95 & $6.499(0.021)$ \\
\hline NGC $3353\left[-6^{\prime \prime},-9^{\prime \prime}\right]$ & & ${ }^{12} \mathrm{CO}(1-0)$ & 4 & 21.4 & 5.2 & 4.6 & 8.8 & 98 & 33 & $0.319(0.037)$ & $0.310(0.036)$ & 1.66 & $6.123(0.051)$ \\
\hline NGC $3353\left[-6^{\prime \prime},-9^{\prime \prime}\right]$ & & ${ }^{12} \mathrm{CO}(1-0)$ & Total & 21.4 & 5.2 & 4.6 & 20.0 & 0 & 78 & $1.701(0.075)$ & $1.652(0.073)$ & 8.86 & $6.850(0.019)$ \\
\hline NGC $3353\left[-6^{\prime \prime},-9^{\prime \prime}\right]$ & & ${ }^{12} \mathrm{CO}(2-1)$ & 1 & 10.7 & 5.2 & 6.1 & 5.9 & -2 & 36 & $0.405(0.190)$ & $0.222(0.104)$ & 4.70 & $5.972(0.204)$ \\
\hline NGC $3353\left[-6^{\prime \prime},-9^{\prime \prime}\right]$ & & ${ }^{12} \mathrm{CO}(2-1)$ & 2 & 10.7 & 5.2 & 6.1 & 13.8 & 23 & 12 & $0.332(0.115)$ & $0.182(0.063)$ & 3.85 & $5.886(0.151)$ \\
\hline NGC $3353\left[-6^{\prime \prime},-9^{\prime \prime}\right]$ & & ${ }^{12} \mathrm{CO}(2-1)$ & Total & 10.7 & 5.2 & 6.1 & 13.8 & 0 & 25 & $0.737(0.223)$ & $0.404(0.122)$ & 8.55 & $6.232(0.131)$ \\
\hline
\end{tabular}

Notes. All temperature units in this table are main-beam, $T_{\mathrm{mb}}$. Values in parentheses are the $1 \sigma$ uncertainties. The total line flux is the sum of the individual components, and the total uncertainty is calculated by adding in quadrature the individual uncertainties. Systemic velocities are heliocentric, taken from NED, and used as the (optical convention) reference in Fig. 1. ${ }^{(a)}$ Offsets of the component's central velocity relative to the systemic one. ${ }^{(b)}$ These have been corrected for beam dilution to a common beam size of $22^{\prime \prime}$ as described in Sect. 4 and Appendix A.

2008 to 2010 as part of the proposals 082.B-0795A, 085.F9320A, and 087.F-9316A. ${ }^{12} \mathrm{CO}(2-1)$ measurements were also obtained for some sources, as reported in Tables 2, 3, and 5. We used the "standard" receiver+backend configurations, APEX-1 (HET230+FTTS1) at $230 \mathrm{GHz}$ and APEX-2 (HET345+FTTS1) at $345 \mathrm{GHz}$, with an intrinsic resolution of $0.12 \mathrm{MHz}$ at both frequencies. The sources were small enough to be observed in one pointing with wobbler switching (in ON/OFF mode); pointing was checked every $2 \mathrm{~h}$, and calibrations were performed every 10 min. Pointing accuracy was typically $\$ 2 \prime$. System temperatures for the ${ }^{12} \mathrm{CO}(3-2)\left({ }^{12} \mathrm{CO}(2-1)\right)$ observations ranged from $\sim 180 \mathrm{~K}$ to $670 \mathrm{~K}(\sim 160 \mathrm{~K}$ to $270 \mathrm{~K})$ with precipitable water vapor from $0.2 \mathrm{~mm}$ to $1.1 \mathrm{~mm}$ (1.2 $\mathrm{mm}$ to $3 \mathrm{~mm})$.

Only NGC 1140 was observed in the ${ }^{12} \mathrm{CO}(4-3)$ and [CI](1-0) transitions (Period 82), following the same observing protocols as for the lower frequencies but with APEX-3 (HET460+FTTS1) at $460 \mathrm{GHz}$ (intrinsic resolution $0.98 \mathrm{MHz}$ ). System temperatures for these higher frequency observations ranged from $\sim 380 \mathrm{~K}$ to $740 \mathrm{~K}(\sim 370 \mathrm{~K}$ to $550 \mathrm{~K})$ for $[\mathrm{CI}](1-0)$ $\left({ }^{12} \mathrm{CO}(4-3)\right)$. Water vapor columns ranged from $0.2 \mathrm{~mm}$ to $0.6 \mathrm{~mm}$ for [CI], and from $0.3 \mathrm{~mm}$ to $0.4 \mathrm{~mm}$ for ${ }^{12} \mathrm{CO}(4-3)$.

\subsection{Data reduction}

We adopted the GILDAS/CLASS data reduction package ${ }^{3}$ to obtain averaged spectra for all transitions. To remove the baseline from each spectrum, a polynomial was fitted to the line-free regions of each scan (defined a priori) and subtracted; the scans were thereafter Hanning smoothed and averaged, and a constant baseline subtracted. We measured the peak intensities, central velocities, full width half-maximum (FWHM) and velocityintegrated fluxes of the detected lines by fitting Gaussian profiles to the data. In most cases, there is significant velocity structure in the emission, so we fit the line profiles to multiple Gaussians. In these cases we use the sum of the integrated line intensities from the multiple Gaussian fits in the analysis.

Antenna temperatures $\left(T_{\mathrm{A}}^{*}\right)$ have been converted to mainbeam brightness temperatures $\left(T_{\mathrm{mb}}\right)$ by dividing the antenna temperatures by $\eta \equiv B_{\text {eff }} / F_{\text {eff }}$, where $B_{\text {eff }}$ and $F_{\text {eff }}$ are the beam and forward hemisphere efficiencies, respectively (i.e., $\left.T_{\mathrm{mb}}=T_{\mathrm{A}}^{*} / \eta\right)$. To convert the measured ${ }^{12} \mathrm{CO}(1-0)$ antenna temperatures, $\left[T_{\mathrm{mb}}(\mathrm{K})\right]$ to fluxes $[S(\mathrm{Jy})]$, we used the standard

\footnotetext{
3 http://www.iram. fr/IRAMFR/GILDAS
} 
Table 3. CO emission line parameters for NGC 7077, UM 448 and UM 462

\begin{tabular}{|c|c|c|c|c|c|c|c|c|c|c|c|c|c|}
\hline Name & $\begin{array}{c}\text { Systemic } \\
\text { velocity } \\
\left(\mathrm{km} \mathrm{s}^{-1}\right)\end{array}$ & Transition & Component & $\begin{array}{c}\text { Beam } \\
\text { size } \\
(") \\
\end{array}$ & $\begin{array}{c}\text { Channel } \\
\text { width } \\
\left(\mathrm{km} \mathrm{s}^{-1}\right) \\
\end{array}$ & $\begin{array}{l}T_{\mathrm{rms}} \\
(\mathrm{mK})\end{array}$ & $\begin{array}{l}T_{\text {peak }} \\
(\mathrm{mK})\end{array}$ & $\begin{array}{l}\text { Offset }^{a} \\
\left(\mathrm{~km} \mathrm{~s}^{-1}\right)\end{array}$ & $\begin{array}{l}F W H M \\
\left(\mathrm{~km} \mathrm{~s}^{-1}\right)\end{array}$ & $\begin{array}{c}I_{\mathrm{CO}} \\
\left(\mathrm{K} \mathrm{km} \mathrm{s}^{-1}\right)\end{array}$ & $\begin{array}{c}I_{\mathrm{CO}}^{\mathrm{cor} b} \\
\left(\mathrm{Km} \mathrm{s}^{-1}\right)\end{array}$ & $\begin{array}{c}S_{\mathrm{CO}}^{\mathrm{cor} b} \\
\left(\mathrm{Jy} \mathrm{km} \mathrm{s}^{-1}\right)\end{array}$ & $\begin{array}{c}\log \left(L_{\mathrm{cor}}^{\prime}\right)^{b} \\
\left(\mathrm{~K} \mathrm{~km} \mathrm{~s}^{-1} \mathrm{pc}^{2}\right)\end{array}$ \\
\hline NGC 7077 & 1152 & ${ }^{12} \mathrm{CO}(1-0)$ & 1 & 21.4 & 5.2 & 1.7 & 11.6 & -5 & 21 & $0.265(0.034)$ & $0.257(0.033)$ & 1.38 & $5.996(0.055)$ \\
\hline NGC 7077 & & ${ }^{12} \mathrm{CO}(1-0)$ & 2 & 21.4 & 5.2 & 1.7 & 6.5 & 36 & 31 & $0.224(0.040)$ & $0.217(0.039)$ & 1.16 & $5.923(0.077)$ \\
\hline NGC 7077 & & ${ }^{12} \mathrm{CO}(1-0)$ & Total & 21.4 & 5.2 & 1.7 & 11.6 & 0 & 41 & $0.489(0.052)$ & $0.474(0.050)$ & 2.54 & $6.262(0.046)$ \\
\hline NGC 7077 & & ${ }^{12} \mathrm{CO}(2-1)$ & 1 & 10.7 & 5.2 & 3.9 & 9.6 & -9 & 29 & $0.578(0.086)$ & $0.298(0.044)$ & 6.30 & $6.055(0.064)$ \\
\hline NGC 7077 & & ${ }^{12} \mathrm{CO}(2-1)$ & 2 & 10.7 & 5.2 & 3.9 & 10.4 & 37 & 23 & $0.485(0.084)$ & $0.250(0.043)$ & 5.28 & $5.979(0.075)$ \\
\hline NGC 7077 & & ${ }^{12} \mathrm{CO}(2-1)$ & Total & 10.7 & 5.2 & 3.9 & 10.4 & 0 & 46 & $1.063(0.120)$ & $0.548(0.062)$ & 11.58 & $6.320(0.049)$ \\
\hline NGC 7077 & & ${ }^{12} \mathrm{CO}(3-2)$ & 1 & 18.1 & 6.8 & 2.8 & 12.1 & -1 & 16 & $0.259(0.057)$ & $0.208(0.046)$ & 9.12 & $5.864(0.096)$ \\
\hline NGC 7077 & & ${ }^{12} \mathrm{CO}(3-2)$ & 2 & 18.1 & 6.8 & 2.8 & 4.2 & 37 & 50 & $0.277(0.092)$ & $0.223(0.074)$ & 9.78 & $5.894(0.143)$ \\
\hline NGC 7077 & & ${ }^{12} \mathrm{CO}(3-2)$ & Total & 18.1 & 6.8 & 2.8 & 12.1 & 0 & 38 & $0.536(0.085)$ & $0.431(0.068)$ & 18.89 & $6.180(0.069)$ \\
\hline UM 448 & 5564 & ${ }^{12} \mathrm{CO}(1-0)$ & 1 & 21.7 & 5.3 & 1.6 & 4.8 & -115 & 46 & $0.106(0.034)$ & $0.104(0.033)$ & 0.54 & $6.934(0.138)$ \\
\hline UM 448 & & ${ }^{12} \mathrm{CO}(1-0)$ & 2 & 21.7 & 5.3 & 1.6 & 6.1 & -59 & 61 & $0.398(0.140)$ & $0.392(0.137)$ & 2.04 & $7.509(0.152)$ \\
\hline UM 448 & & ${ }^{12} \mathrm{CO}(1-0)$ & 3 & 21.7 & 5.3 & 1.6 & 10.9 & 0 & 46 & $0.547(0.125)$ & $0.539(0.123)$ & 2.80 & $7.647(0.099)$ \\
\hline UM 448 & & ${ }^{12} \mathrm{CO}(1-0)$ & Total & 21.7 & 5.3 & 1.6 & 10.9 & 0 & 115 & $1.051(0.190)$ & $1.035(0.187)$ & 5.38 & $7.931(0.079)$ \\
\hline UM 448 & & ${ }^{12} \mathrm{CO}(2-1)$ & 1 & 10.9 & 5.3 & 4.0 & 3.8 & -145 & 71 & $0.212(0.093)$ & $0.105(0.046)$ & 2.16 & $6.932(0.190)$ \\
\hline UM 448 & & ${ }^{12} \mathrm{CO}(2-1)$ & 2 & 10.9 & 5.3 & 4.0 & 3.7 & -95 & 52 & $0.407(0.118)$ & $0.202(0.058)$ & 4.15 & $7.216(0.126)$ \\
\hline UM 448 & & ${ }^{12} \mathrm{CO}(2-1)$ & 3 & 10.9 & 5.3 & 4.0 & 12.3 & -15 & 71 & $1.871(0.099)$ & $0.930(0.049)$ & 19.10 & $7.879(0.023)$ \\
\hline UM 448 & & ${ }^{12} \mathrm{CO}(2-1)$ & Total & 10.9 & 5.3 & 4.0 & 12.3 & 0 & 130 & $2.490(0.179)$ & $1.237(0.089)$ & 25.41 & $8.003(0.031)$ \\
\hline UM 448 & & ${ }^{12} \mathrm{CO}(2-1)$ & 1 & 27.6 & 10.4 & 2.1 & 8.9 & -47 & 22 & $0.153(0.081)$ & $0.208(0.111)$ & 3.98 & $7.198(0.232)$ \\
\hline UM 448 & & ${ }^{12} \mathrm{CO}(2-1)$ & 2 & 27.6 & 10.4 & 2.1 & 6.7 & -45 & 118 & $0.614(0.212)$ & $0.839(0.290)$ & 16.03 & $7.803(0.150)$ \\
\hline UM 448 & & ${ }^{12} \mathrm{CO}(2-1)$ & 3 & 27.6 & 10.4 & 2.1 & 12.1 & 34 & 82 & $0.776(0.191)$ & $1.059(0.261)$ & 20.25 & $7.904(0.107)$ \\
\hline UM 448 & & ${ }^{12} \mathrm{CO}(2-1)$ & Total & 27.6 & 10.4 & 2.1 & 12.1 & 0 & 81 & $1.543(0.297)$ & $2.106(0.406)$ & 40.27 & $8.202(0.084)$ \\
\hline UM 448 & & ${ }^{12} \mathrm{CO}(3-2)$ & 1 & 18.4 & 13.8 & 1.6 & 3.9 & -70 & 22 & $0.111(0.042)$ & $0.090(0.034)$ & 3.82 & $6.827(0.165)$ \\
\hline UM 448 & & ${ }^{12} \mathrm{CO}(3-2)$ & 2 & 18.4 & 13.8 & 1.6 & 4.2 & -27 & 23 & $0.126(0.069)$ & $0.101(0.056)$ & 4.32 & $6.881(0.238)$ \\
\hline UM 448 & & ${ }^{12} \mathrm{CO}(3-2)$ & 3 & 18.4 & 13.8 & 1.6 & 6.7 & 33 & 72 & $0.637(0.049)$ & $0.513(0.039)$ & 21.88 & $7.585(0.033)$ \\
\hline UM 448 & & ${ }^{12} \mathrm{CO}(3-2)$ & Total & 18.4 & 13.8 & 1.6 & 6.7 & 0 & 103 & $0.875(0.094)$ & $0.705(0.076)$ & 30.02 & $7.723(0.047)$ \\
\hline UM 462 & 1057 & ${ }^{12} \mathrm{CO}(1-0)$ & Total & 21.4 & 15.7 & 2.5 & 6.7 & -8 & 123 & $0.872(0.157)$ & $0.872(0.157)$ & 4.43 & $6.613(0.078)$ \\
\hline UM 462 & & ${ }^{12} \mathrm{CO}(2-1)$ & Total & 10.7 & 15.7 & 4.8 & - & - & - & $<0.87$ & $0.012(0.101)$ & - & $<5.72$ \\
\hline UM 462 & & ${ }^{12} \mathrm{CO}(3-2)$ & Total & 18.1 & 13.6 & 1.6 & - & - & - & $<0.87$ & - & - & $<5.78$ \\
\hline
\end{tabular}

Notes. All temperature units in this table are main-beam, $T_{\mathrm{mb}}$. Values in parentheses are the $1 \sigma$ uncertainties. The total line flux is the sum of the individual components, and the total uncertainty is calculated by adding in quadrature the individual uncertainties. Systemic velocities are heliocentric, taken from NED, and used as the (optical convention) reference in Fig. 1 ( $^{(a)}$ Offsets of the component's central velocity relative to the systemic one. ${ }^{(b)}$ These have been corrected for beam dilution to a common beam size of $22^{\prime \prime}$ as described in Sect. 4 and Appendix A.

Table 4. Emission line parameters for NGC 1140.

\begin{tabular}{|c|c|c|c|c|c|c|c|c|c|c|c|c|}
\hline Name & Transition & Component & $\begin{array}{c}\text { Beam } \\
\text { size } \\
\left({ }^{\prime \prime}\right) \\
\end{array}$ & $\begin{array}{c}\text { Channel } \\
\text { width } \\
\left(\mathrm{km} \mathrm{s}^{-1}\right)\end{array}$ & $\begin{array}{c}T_{\mathrm{rms}} \\
(\mathrm{mK})\end{array}$ & $\begin{array}{l}T_{\text {peak }} \\
(\mathrm{mK})\end{array}$ & $\begin{array}{l}\text { Offset }^{a} \\
\left(\mathrm{~km} \mathrm{~s}^{-1}\right)\end{array}$ & $\begin{array}{l}F W H M \\
\left(\mathrm{~km} \mathrm{~s}^{-1}\right)\end{array}$ & $\begin{array}{c}I_{\mathrm{CO}} \\
\left(\mathrm{K} \mathrm{km} \mathrm{s}^{-1}\right)\end{array}$ & $\begin{array}{c}I_{\mathrm{CO}}^{\mathrm{cor} b} \\
\left(\mathrm{~K} \mathrm{~km} \mathrm{~s}^{-1}\right)\end{array}$ & $\begin{array}{c}S_{\mathrm{CO}}^{\mathrm{cor} b} \\
\left(\mathrm{Jy} \mathrm{km} \mathrm{s}^{-1}\right)\end{array}$ & $\begin{array}{c}\log \left(L_{\text {cor }}^{\prime}\right)^{b} \\
\left(\mathrm{~K} \mathrm{~km} \mathrm{~s}^{-1} \mathrm{pc}^{2}\right)\end{array}$ \\
\hline NGC 1140 & ${ }^{12} \mathrm{CO}(1-0)$ & 1 & 21.4 & 5.2 & 1.6 & 9.5 & -21 & 20 & $0.205(0.016)$ & $0.200(0.015)$ & 1.07 & $6.004(0.033)$ \\
\hline NGC 1140 & ${ }^{12} \mathrm{CO}(1-0)$ & 2 & 21.4 & 5.2 & 1.6 & 7.8 & 0 & 24 & $0.201(0.016)$ & $0.196(0.015)$ & 1.05 & $5.996(0.034)$ \\
\hline NGC 1140 & ${ }^{12} \mathrm{CO}(1-0)$ & 3 & 21.4 & 5.2 & 1.6 & 4.5 & 35 & 25 & $0.123(0.016)$ & $0.120(0.015)$ & 0.64 & $5.782(0.055)$ \\
\hline NGC 1140 & ${ }^{12} \mathrm{CO}(1-0)$ & 4 & 21.4 & 5.2 & 1.6 & 5.4 & 64 & 8 & $0.049(0.016)$ & $0.048(0.015)$ & 0.26 & $5.386(0.138)$ \\
\hline NGC 1140 & ${ }^{12} \mathrm{CO}(1-0)$ & Total & 21.4 & 5.2 & 1.6 & 9.5 & 0 & 85 & $0.578(0.031)$ & $0.564(0.031)$ & 3.02 & $6.455(0.024)$ \\
\hline NGC 1140 & ${ }^{12} \mathrm{CO}(2-1)$ & 1 & 10.7 & 5.2 & 5.0 & 6.0 & -71 & 20 & $0.212(0.047)$ & $0.126(0.028)$ & 2.65 & $5.797(0.096)$ \\
\hline NGC 1140 & ${ }^{12} \mathrm{CO}(2-1)$ & 2 & 10.7 & 5.2 & 5.0 & 17.0 & -17 & 37 & $1.124(0.047)$ & $0.668(0.028)$ & 14.10 & $6.522(0.018)$ \\
\hline NGC 1140 & ${ }^{12} \mathrm{CO}(2-1)$ & 3 & 10.7 & 5.2 & 5.0 & 8.5 & 10 & 25 & $0.389(0.047)$ & $0.231(0.028)$ & 4.88 & $6.061(0.052)$ \\
\hline NGC 1140 & ${ }^{12} \mathrm{CO}(2-1)$ & 4 & 10.7 & 5.2 & 5.0 & 6.4 & 38 & 23 & $0.260(0.047)$ & $0.155(0.028)$ & 3.26 & $5.886(0.078)$ \\
\hline NGC 1140 & ${ }^{12} \mathrm{CO}(2-1)$ & Total & 10.7 & 5.2 & 5.0 & 17.0 & 0 & 109 & $1.985(0.093)$ & $1.180(0.056)$ & 24.89 & $6.769(0.020)$ \\
\hline NGC 1140 & ${ }^{12} \mathrm{CO}(3-2)$ & 1 & 18.1 & 6.8 & 3.6 & 20.0 & -30 & 27 & $0.686(0.182)$ & $0.577(0.153)$ & 25.24 & $6.423(0.115)$ \\
\hline NGC 1140 & ${ }^{12} \mathrm{CO}(3-2)$ & 2 & 18.1 & 6.8 & 3.6 & 11.0 & 0 & 22 & $0.308(0.171)$ & $0.259(0.144)$ & 11.33 & $6.075(0.241)$ \\
\hline NGC 1140 & ${ }^{12} \mathrm{CO}(3-2)$ & 3 & 18.1 & 6.8 & 3.6 & 9.9 & 47 & 13 & $0.162(0.044)$ & $0.136(0.037)$ & 5.96 & $5.796(0.117)$ \\
\hline NGC 1140 & ${ }^{12} \mathrm{CO}(3-2)$ & Total & 18.1 & 6.8 & 3.6 & 20.0 & 0 & 77 & $1.155(0.252)$ & $0.972(0.212)$ & 42.52 & $6.650(0.095)$ \\
\hline NGC 1140 & ${ }^{12} \mathrm{CO}(4-3)$ & 1 & 13.6 & 10.2 & 7.5 & 18.2 & -37 & 23 & $0.645(0.168)$ & $0.440(0.114)$ & 33.11 & $6.291(0.113)$ \\
\hline NGC 1140 & ${ }^{12} \mathrm{CO}(4-3)$ & 2 & 13.6 & 10.2 & 7.5 & 9.2 & -1 & 14 & $0.209(0.132)$ & $0.142(0.090)$ & 10.71 & $5.801(0.274)$ \\
\hline NGC 1140 & ${ }^{12} \mathrm{CO}(4-3)$ & Total & 13.6 & 10.2 & 7.5 & 18.2 & 0 & 38 & $0.854(0.213)$ & $0.582(0.146)$ & 43.82 & $6.413(0.109)$ \\
\hline NGC 1140 & ${ }^{13} \mathrm{CO}(1-0)$ & Total & 22.4 & 10.9 & 0.6 & 2.1 & 2 & 23 & $0.052(0.016)$ & $0.053(0.016)$ & 0.26 & $5.423(0.131)$ \\
\hline NGC 1140 & ${ }^{13} \mathrm{CO}(2-1)$ & 1 & 11.2 & 5.5 & 0.9 & 1.3 & -21 & 41 & $0.093(0.020)$ & $0.057(0.012)$ & 1.09 & $5.450(0.093)$ \\
\hline NGC 1140 & ${ }^{13} \mathrm{CO}(2-1)$ & 2 & 11.2 & 5.5 & 0.9 & 0.8 & 74 & 58 & $0.079(0.021)$ & $0.048(0.013)$ & 0.93 & $5.381(0.117)$ \\
\hline NGC 1140 & ${ }^{13} \mathrm{CO}(2-1)$ & Total & 11.2 & 5.5 & 0.9 & 1.3 & 0 & 95 & $0.172(0.029)$ & $0.105(0.018)$ & 2.02 & $5.718(0.073)$ \\
\hline NGC 1140 & $\mathrm{C} 18 \mathrm{O}(1-0)$ & Total & 22.5 & 11.0 & 0.5 & - & - & - & $<0.10$ & - & - & $<5.40$ \\
\hline NGC 1140 & $\mathrm{HCN}(1-0)$ & Total & 21.8 & 12.8 & 0.5 & - & - & - & $<0.10$ & - & - & $<5.44$ \\
\hline NGC 1140 & $\mathrm{CN}(1-0)$ & Total & 21.8 & 10.6 & 1.2 & - & - & - & $<0.10$ & - & - & $<5.75$ \\
\hline NGC 1140 & $\mathrm{CS}(2-1)$ & Total & 25.2 & 12.3 & 0.6 & - & - & - & $<0.10$ & - & - & $<5.64$ \\
\hline NGC 1140 & {$[\mathrm{CI}](1-0)$} & Total & 12.7 & 9.6 & 5.7 & 6.8 & 5 & 51 & $0.563(0.188)$ & $0.369(0.123)$ & 31.63 & $6.215(0.145)$ \\
\hline
\end{tabular}

Notes. All temperature units in this table are main-beam, $T_{\mathrm{mb}}$. Values in parentheses are the $1 \sigma$ uncertainties. The total line flux is the sum of the individual components, and the total uncertainty is calculated by adding in quadrature the individual uncertainties. We assumed a (heliocentric) systemic velocity of $1501 \mathrm{~km} \mathrm{~s}^{-1}$ (from NED) for NGC 1140, and used this as the (optical convention) reference velocity in Fig. $2 .{ }^{(a)}$ Offsets of the component's central velocity relative to the systemic one. ${ }^{(b)}$ These have been corrected for beam dilution to a common beam size of $22^{\prime \prime}$ as described in Sect. 4 and Appendix A. 
Table 5. $3 \sigma$ upper limits of undetected emission lines for II Zw 40 and SBS 0335-052.

\begin{tabular}{lccrrrr}
\hline \hline \multicolumn{1}{c}{ Name } & Transition & Component & $\begin{array}{c}\text { Beam } \\
\text { size }\left({ }^{\prime \prime}\right)\end{array}$ & $\begin{array}{c}\text { Channel } \\
\text { width }\left(\mathrm{km} \mathrm{s}^{-1}\right)\end{array}$ & $\begin{array}{c}T_{\text {rms }} \\
(\mathrm{mK})\end{array}$ & $\begin{array}{c}I_{\mathrm{CO}} \\
\left(\mathrm{K} \mathrm{km} \mathrm{s}^{-1}\right)\end{array}$ \\
\hline II Zw 40 & ${ }^{12} \mathrm{CO}(2-1)$ & Total & 27.1 & 10.2 & 2.5 & $<0.23$ \\
II Zw 40 & ${ }^{12} \mathrm{CO}(3-2)$ & Total & 18.1 & 13.6 & 1.9 & $<0.23$ \\
& & & & & & \\
SBS0335-052 & ${ }^{12} \mathrm{CO}(2-1)$ & Total & 27.4 & 10.3 & 2.2 & $<0.20$ \\
SBS0335-052 & ${ }^{12} \mathrm{CO}(3-2)$ & Total & 18.3 & 13.7 & 2.7 & $<0.33$ \\
\hline
\end{tabular}

Notes. All temperature units in this table are main-beam, $T_{\mathrm{mb}}$.

conversion factors for both telescopes. The ${ }^{12} \mathrm{CO}(1-0),{ }^{12} \mathrm{CO}(2-$ $1)$, and ${ }^{12} \mathrm{CO}(3-2)$ line profiles for the galaxies in which $\mathrm{CO}$ was detected are shown in Fig. 1, together with the Gaussian fits obtained using the GILDAS/CLASS package as described above and reported in Tables 2-4. The upper limits for II Zw 40 and SBS 0335-052 are given in Table 5. Limits for non-detections are $3 \sigma$, taking the width of three contiguous velocity channels and the rms noise in line-free regions of the spectrum.

For the total flux in each transition, we have included the different velocity components in each galaxy into a global, spectrally-integrated sum. While some of the spectra show slightly different profiles in the different transitions, the signalto-noise $(\mathrm{S} / \mathrm{N})$ is insufficient to allow an analysis of the separate components. Although a few of the individual components may be too narrow to be significant (because their width is smaller than three velocity channels, e.g., Mrk 996, NGC 3353, NGC 1140, see Figs. 1, 2), we have checked that the total intensities are robust to the details of the fitting by repeating the fits with different numbers of components. Some of the measured values in the tables differ slightly from those given in Paper I; this is because we have redone the data reduction for all transitions in order to render the results as consistent as possible. The main difference is the newer version of CLASS used here that seems to give integrated fluxes which can differ by a few percent (but within the error limits) with respect to those in Paper I (performed with an older version of CLASS).

In Paper I, for the galaxies with the largest apparent sizes, to estimate total ${ }^{12} \mathrm{CO}(1-0)$ flux beyond even the largest beam we applied aperture corrections. Here they are not adopted because we prefer to correct the observations to a common beam size, rather than also extrapolating to an uncertain total flux.

\subsection{Comparison with previous observations}

A detailed comparison of our ${ }^{12} \mathrm{CO}(1-0)$ measured intensities relative to previous work is given in Paper I. Here we compare only the higher- $J$ CO transitions with published literature values. Sage et al. (1992) observed II Zw 40, NGC 3353 (Haro 3), NGC 7077, and UM 462 in ${ }^{12} \mathrm{CO}(2-1)$, and securely detected only the first three. Like the ${ }^{12} \mathrm{CO}(1-0)$ measurements, their results from the IRAM $30 \mathrm{~m}$ for ${ }^{12} \mathrm{CO}(2-1)$ in NGC 3353 are in good agreement with the line parameters deduced from our central pointing. For NGC 7077 , our ${ }^{12} \mathrm{CO}(2-1)$ flux of $1.06 \mathrm{~K} \mathrm{~km} \mathrm{~s}^{-1}$ is $\gtrsim 50 \%$ larger than the value of $0.68 \mathrm{~K} \mathrm{~km} \mathrm{~s}^{-1}$ reported by Sage et al. (1992); given the similar uncertainties, it is difficult to understand the discrepancy, especially since our ${ }^{12} \mathrm{CO}(1-0)$ flux is smaller than theirs by $\sim 30 \%$. Our ${ }^{12} \mathrm{CO}(2-1)$ non-detection for UM 462 is consistent with the non-detection by Sage et al. (1992), while our upper limit is smaller. Instead, our non-detection with APEX of ${ }^{12} \mathrm{CO}(2-1)$ in II Zw 40 is inconsistent with Sage et al. (1992); at the flux level found by them, we should have detected this galaxy, as our $3 \sigma$ upper limit is several times smaller.

NGC 1140 and II Zw 40 were also observed previously in ${ }^{12} \mathrm{CO}(2-1)$ with the IRAM $30 \mathrm{~m}$ by Albrecht et al. (2004). Although the 2-1/1-0 line ratios for NGC 1140 are similar, they find $\sim 40-60 \%$ larger intensities in both lines. The line intensity they report for the IRAM 30-m spectrum of II Zw 40 (UGCA 116) is larger than our APEX upper limit, although less than half of the $30 \mathrm{~m}$ detection reported by Sage et al. (1992). The peak ${ }^{12} \mathrm{CO}(3-2)$ temperatures observed with ALMA in the main knots in II Zw 40 by Kepley et al. (2016) exceed those of our upper limits; but beam dilution is probably playing an important role since the ALMA beam is $\sim 0^{\prime \prime} .5$ compared to the APEX beam of $\sim 18^{\prime \prime}$.

One of our targets, NGC 7077, was observed by Mao et al. (2010). The ratio of ${ }^{12} \mathrm{CO}(3-2)$ to ${ }^{12} \mathrm{CO}(1-0)$ we find for this galaxy of 1.1 is in excellent agreement with their study.

In conclusion, there is no clear systematic difference between our results and previous work. Nevertheless, the formal uncertainties given in Tables $2-5$ should probably be considered as lower limits to the true uncertainties.

\section{Correction to a common beam size}

The main difficulty in the interpretation of line ratios from single-dish observations is the correction for different beam sizes. The physical models we discuss in Sect. 7 rely on brightness temperatures $T_{\mathrm{B}}$ within a common beam size, while we observe main-beam brightness temperatures, $T_{\mathrm{mb}}$, within different beams. To correct for this, we have pursued two independent approaches:

(1) Used maps of the cool dust emission $(\sim 160 \mu \mathrm{m})$ to scale the different transitions to a common beam size of $22^{\prime \prime}$, with the underlying assumption that the molecular emission is distributed like the cool dust. The PACS $160 \mu \mathrm{m}$ maps are particularly well suited to such an endeavor because their intrinsic FWHM is $\sim 11^{\prime \prime}$, similar to the smallest beam size in our observations $\left({ }^{12} \mathrm{CO}(2-1), 10^{\prime \prime} \cdot 7\right)$.

(2) Assumed that the molecular emission follows a Gaussian distribution with a FWHM size to be determined by letting it vary in the fit to the line intensities. This can be done in a robust way only for NGC 1140 where we have a sufficient number of line intensities to constrain the fit.

Observations at high spatial resolution $(\$ 2 \mathrm{pc})$ of metal-poor galaxies in the Local Group suggest that the $\mathrm{CO}$ clouds are more concentrated than the dust emission (e.g., Rubio et al. 2015; Schruba et al. 2017); if this were true also on the spatial scales probed by our observations ( $\gtrsim 500$ times larger), then our assumption that the gas follows the dust would be incorrect. Thus, analyzing beam correction factors under the assumption of a 

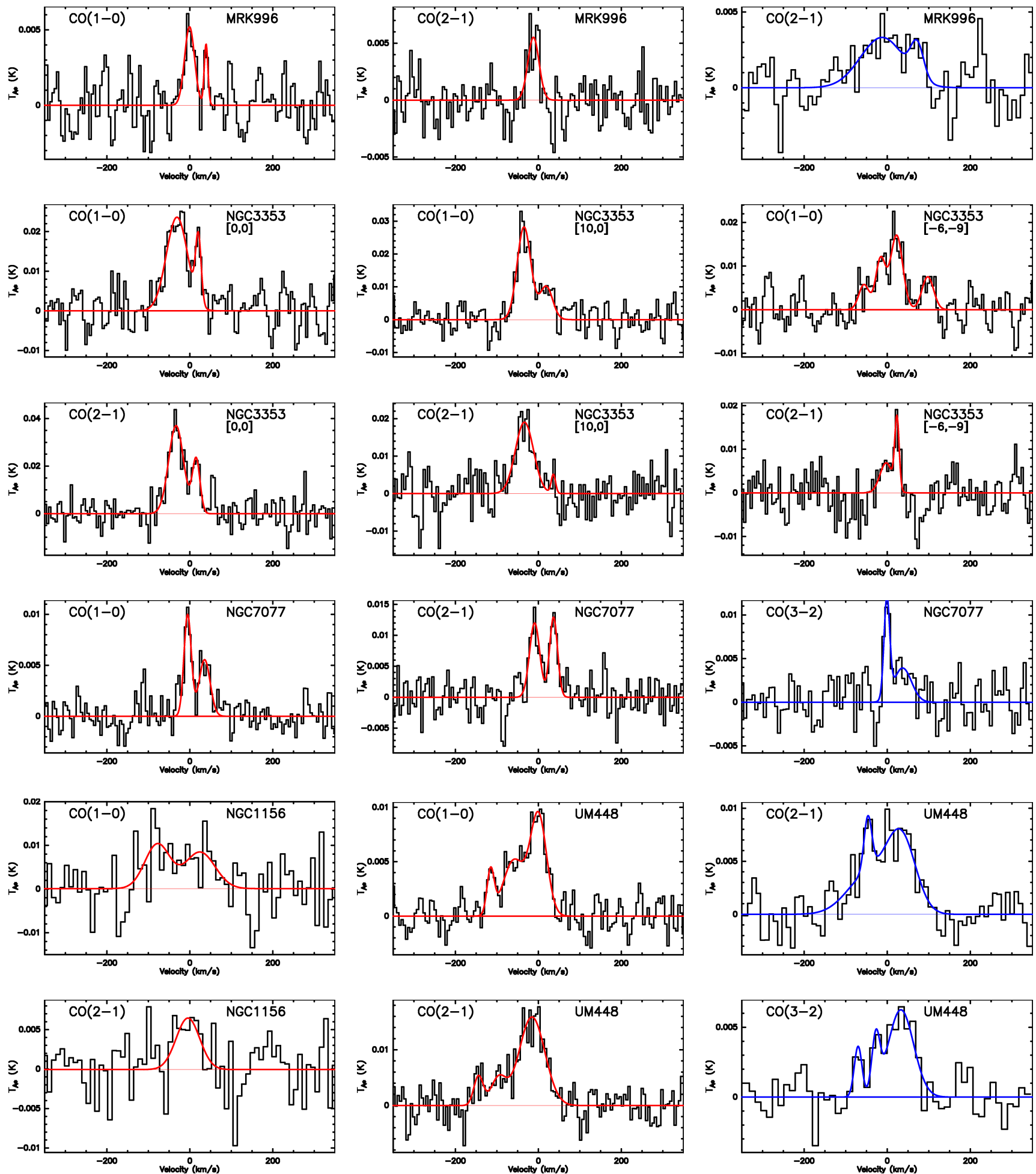

Fig. 1. Various ${ }^{12} \mathrm{CO}$ transitions for Mrk 996, NGC 3353, NGC 7077, NGC 1156, and UM 448. Top row: ${ }^{12} \mathrm{CO}(1-0)$, ${ }^{12} \mathrm{CO}(2-1)$ (IRAM), and ${ }^{12} \mathrm{CO}(2-1)$ (APEX) for Mrk 996; second row: ${ }^{12} \mathrm{CO}(1-0)$ for NGC 3353 (Haro 3) for three pointings $\left([0,0],\left[0,+10^{\prime \prime}\right],\left[-6 ",-9{ }^{\prime \prime}\right]\right)$; third row: ${ }^{12} \mathrm{CO}(2-1)$ for NGC 3353 (Haro 3) for three pointings; fourth row: ${ }^{12} \mathrm{CO}(1-0),{ }^{12} \mathrm{CO}(2-1)$ (IRAM), and ${ }^{12} \mathrm{CO}(3-2)$ (APEX) for NGC 7077 (Mrk 900); fifth row: ${ }^{12} \mathrm{CO}(1-0)$ for NGC 1156 and UM 448, and ${ }^{12} \mathrm{CO}(2-1)$ (APEX) for UM 448; bottom row: ${ }^{12} \mathrm{CO}(2-1)$ (IRAM) for NGC 1156 and UM448 and ${ }^{12} \mathrm{CO}(3-2)$ (APEX) for UM 448. The baselines are shown as a horizontal solid line, together with the multiple-component Gaussian fits as described in Sect. 3.3. The vertical axes are in $T_{\mathrm{A}}^{*}$ units, while in Tables 2 and 3 the units are $T_{\mathrm{mb}}$, converted from $T_{\mathrm{A}}^{*}$ as described in Sect. 3.3. Velocity channels are as reported in Table 2. The red curves and lines refer to 30-m spectra, while the blue ones to APEX.

Gaussian distribution enables an independent assessment of the validity of the inferred beam corrections.
Appendix A describes the formulation of the correction to a common beam size assuming that the molecular emission 

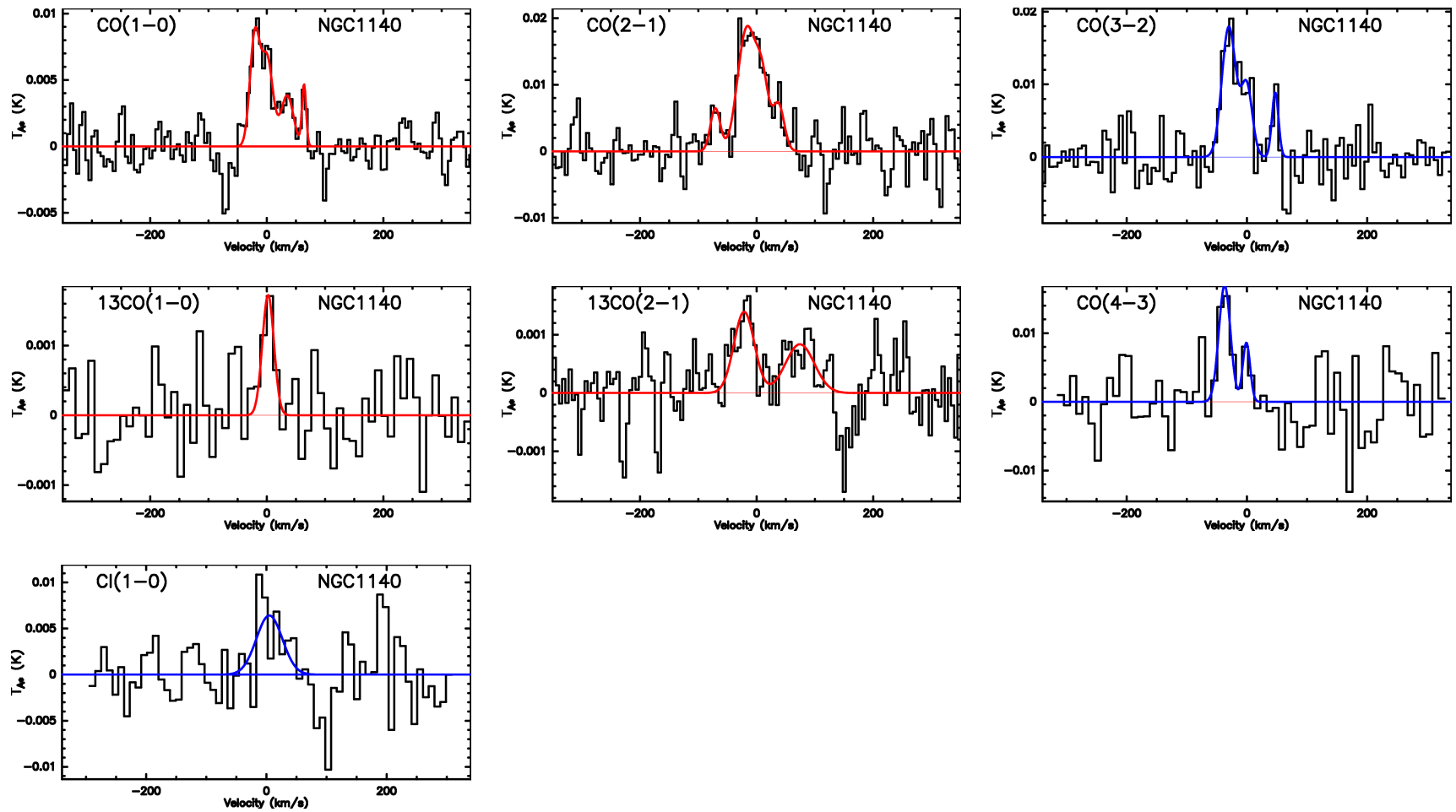

Fig. 2. ${ }^{12} \mathrm{CO},{ }^{13} \mathrm{CO}$, and $[\mathrm{CI}](1-0)$ transitions for $\mathrm{NGC} 1140$. Top row: ${ }^{12} \mathrm{CO}(1-0),{ }^{12} \mathrm{CO}(2-1)$ (IRAM), and ${ }^{12} \mathrm{CO}(3-2)(\mathrm{APEX})$; second row: ${ }^{13} \mathrm{CO}(1-0),{ }^{13} \mathrm{CO}(2-1)$ (IRAM), and ${ }^{12} \mathrm{CO}(4-3)$ (APEX); third row: [CI](1-0) (APEX). As in Fig. 1, the baselines are shown as a horizontal solid line, together with the multiple-component Gaussian fits as described in Sect. 3.3. The vertical axes are in $T_{\mathrm{A}}^{*}$ units, while in Table 4 the units are $T_{\mathrm{mb}}$, converted from $T_{\mathrm{A}}^{*}$ as described in Sect. 3.3. Velocity channels are as reported in Table 2. The red curves and lines refer to 30-m spectra, while the blue ones to APEX.

follows the dust. In brief, we have established that the distribution of the cool dust emission in our targets is well described by an exponential distribution by extracting azimuthally-averaged radial brightness profiles; they are all well fit by an exponential. Moreover, for our targets, $160 \mu \mathrm{m}$ emission is longward of the spectral energy distribution peak, thus tracing as well as possible the cool dust component. We have checked that free-free emission is unimportant at $160 \mu \mathrm{m}$ by inferring its amplitude from the observed SFR (Paper I); for all galaxies for which we have dust images, the expected free-free component is $\lesssim 0.1 \%$ of the observed emission.

We performed aperture photometry to obtain growth curves, in order to approximate the cumulative flux distribution we expect for the molecular gas in the different beams. Finally, we have followed an analytical approach to fit the growth curves, in order to derive the corrections to the expected flux in different apertures. Table A.1 gives the multiplicative correction factors, and the corrected velocity-integrated $T_{\mathrm{mb}}$ values that we will use throughout the paper unless otherwise noted; corrected intensities are also given in the last three columns in Tables $2-4$. While high- $J$ CO emission may be more compact than the gas traced by the lower- $J$ lines, the different radial distributions are expected to be a second-order effect; our proposed correction provides the bulk of the "first-order" correction.

Appendix B discusses the form of the growth curves for a Gaussian source distribution. The main difficulty with applying the Gaussian assumption is the unknown source size; while the source extent can be measured from the analysis of the dust emission, there is no similar way to assess the size of a Gaussian distribution. Thus, when we estimate the physical conditions in the molecular gas by fitting the line intensities (for NGC 1140, the only source for which this is possible), we use the fitting procedure itself to define the source size. The expectation is that because of the similarity of some of the beams in our observations (e.g., ${ }^{12} \mathrm{CO}(2-1)$ with IRAM, ${ }^{12} \mathrm{CO}(4-3)$ with APEX), the fit will be sensitive to the beam correction. This turns out to be the case as will be described in Sect. 7.3.

Mrk 996 shows a markedly broader ${ }^{12} \mathrm{CO}(2-1)$ profile in the larger $\left(27^{\prime \prime}\right)$ APEX beam, relative to the IRAM one $\left(11^{\prime \prime}\right)$. This could be an illustration of the spatial extension of the ${ }^{12} \mathrm{CO}(2-1)$ emitting gas, because of the broader velocity range in the bigger beam; on the other hand, the APEX spectrum is rather noisy, so its reliability is uncertain. Nevertheless, the optical diameter of Mrk 996 is 36" so it is conceivable that there is more CO emission in the larger APEX beam. UM 448 was also observed in ${ }^{12} \mathrm{CO}(2-1)$ with both the $30 \mathrm{~m}$ and APEX, but in this galaxy the shape and breadth of the two profiles are more similar, possibly because the galaxy is smaller, $24^{\prime \prime}$ in diameter.

\section{Empirical line ratios}

Before describing our models of the molecular gas, here we discuss three commonly used diagnostic ratios, ${ }^{12} \mathrm{CO}(2-$ $1) /{ }^{12} \mathrm{CO}(1-0),{ }^{12} \mathrm{CO}(3-2) /{ }^{12} \mathrm{CO}(1-0)$, and $[\mathrm{CI}](1-0) /{ }^{12} \mathrm{CO}(1-0)$. These ratios are calculated using velocity-integrated main-beam temperature, $\int T_{\mathrm{mb}} \mathrm{d} v$ (see Tables 2-4).

\section{1. $\mathrm{CO}(2-1) / \mathrm{CO}(1-0)$}

The ${ }^{12} \mathrm{CO}(2-1)$ transition traces slightly warmer (upper level equivalent temperature $\sim 17 \mathrm{~K}$ ) and denser (critical density $\sim 10^{4} \mathrm{~cm}^{-3}$ ) gas than the $1-0$ transition. In most galaxies, CO emitting gas is optically thick and the observed $\mathrm{CO}(2-1) / \mathrm{CO}(1-$ $0)$ ratios are $\lesssim 1$ (e.g., Braine \& Combes 1992). Figure 3 shows the distribution of the ${ }^{12} \mathrm{CO}(2-1) /{ }^{12} \mathrm{CO}(1-0)$ line temperature ratios $\left(R_{21}\right)$ of our low-metallicity targets together with $R_{21}$ ratios (corrected for beam dilution) of spiral galaxies from 


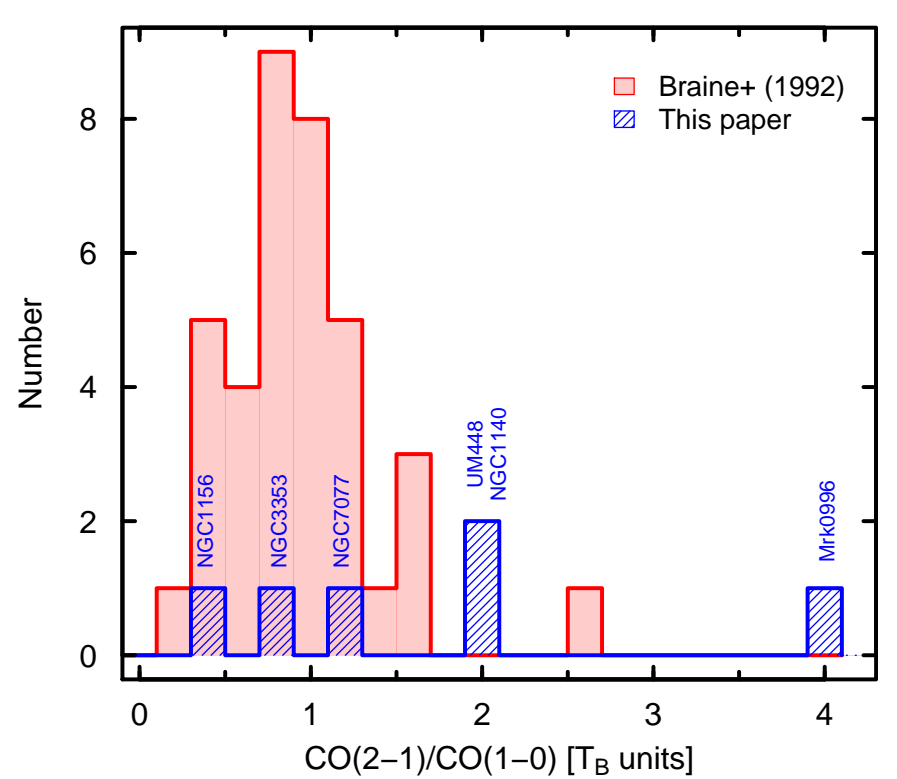

Fig. 3. Histogram of observed ${ }^{12} \mathrm{CO}(2-1) /{ }^{12} \mathrm{CO}(1-0)$ line ratios for our targets corrected for beam dilution as described in Sect. 4. Because of the larger beam, for Mrk 996 and UM 448, we show the APEX ${ }^{12} \mathrm{CO}(2-$ 1) measurements rather than the ones from IRAM. Also plotted are the line ratios corrected for beam dilution of a large sample of spiral galaxies observed by Braine \& Combes (1992).

Braine \& Combes (1992). In Fig. 3, our data are corrected to a common beam size using the exponential approach described in Sect. 4 and in Appendix A.

Even after correction for beam dilution, half of our targets have $R_{21} \gtrsim 2$, higher than most of the normal spirals studied by Braine \& Combes (1992). Braine \& Combes (1992) found an extremely high ratio for another sub-solar metallicity starburst, NGC 3310 with $R_{21} \sim 2.6$; however Zhu et al. (2009) were unable to confirm this, finding a somewhat lower ratio $\left(R_{21} \sim 1.5\right)$, after convolving the observations to a common beam size through their maps.

Although it is tempting to claim that the high $R_{21}$ observed in some of these dwarf galaxies is due to optically thin gas, the $R_{21}$ values are somewhat uncertain because of the beam correction. First, the ratios are dependent on the kind of correction applied. The corrections are sensitive both to the source distribution (exponential versus Gaussian) and to the source size (exponential scale length versus Gaussian FWHM); since we have no way of accurately determining a Gaussian FWHM, we cannot assess how much the $R_{21}$ values would change under another assumption for source distribution. Second, if we use the IRAM ${ }^{12} \mathrm{CO}(2-1)$ observations (with a smaller beam) for Mrk 996 and UM 448, we obtain beam-corrected $R_{21} \sim 0.6$ for Mrk 996, and $R_{21} \sim 1.2$ for UM 448. Thus the high $R_{21}$ values are not altogether robust. The only galaxy in our sample for which we are fairly certain that the true $R_{21}$ value is $\gtrsim 2$ is NGC 1140 . The interpretation of $R_{21}$ for this galaxy will be discussed in Sect. 7.3 where we present physical models for the molecular gas.

\section{2. $\mathrm{CO}(3-2) / \mathrm{CO}(1-0)$}

Figure 4 shows the distribution of the ratio of ${ }^{12} \mathrm{CO}(3-$ $2) /{ }^{12} \mathrm{CO}(1-0)\left(R_{31}\right.$ in $T_{\mathrm{mb}}$ units) for the three sources in our sample with ${ }^{12} \mathrm{CO}(3-2)$ detections, compared to the large sample of Mao et al. (2010). Unlike for the ${ }^{12} \mathrm{CO}(2-1) /{ }^{12} \mathrm{CO}(1-0)$ ratio

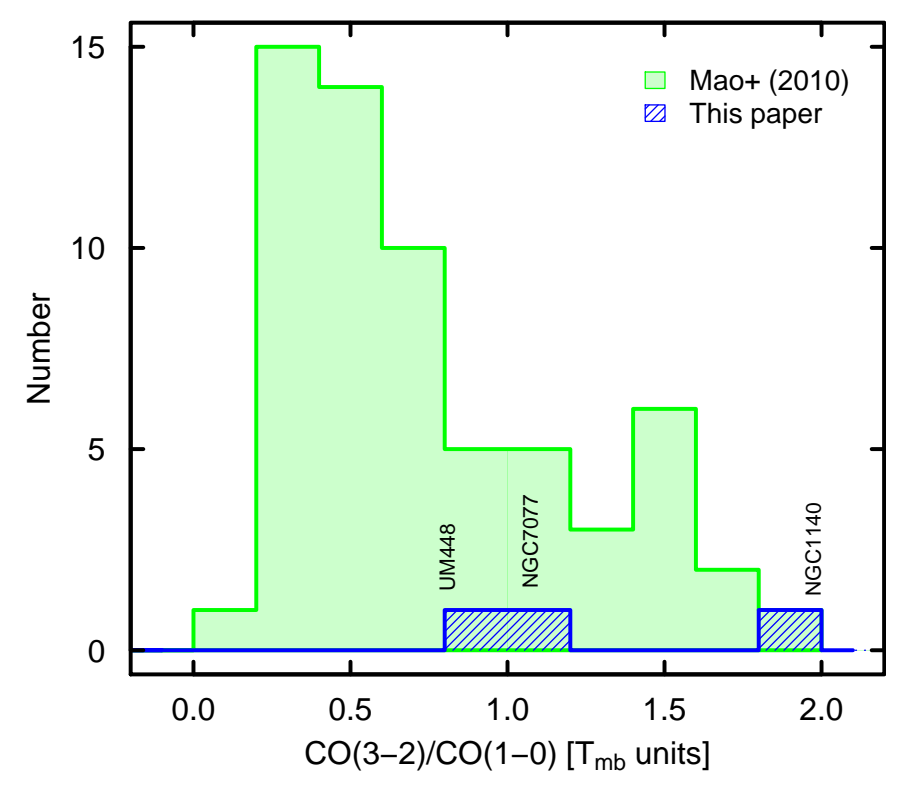

Fig. 4. Histogram of observed ${ }^{12} \mathrm{CO}(3-2) /{ }^{12} \mathrm{CO}(1-0)$ line ratios for our targets. Also shown are the line ratios for a large sample of galaxies observed by Mao et al. (2010). Neither set of observations has been corrected for beam dilution; see Sect. 5.2 for more discussion.

observed with the IRAM $30 \mathrm{~m}$, the APEX beam is $\sim 18^{\prime \prime}$, similar to the IRAM 30-m beam of 22" for the 1-0 line; the Mao et al. (2010) observations were obtained with the Heinrich Hertz Telescope with a ${ }^{12} \mathrm{CO}(3-2)$ beam of $\sim 22^{\prime \prime}$. Thus, we have not applied any correction for beam dilution; the $R_{31}$ values shown in Fig. 4 should be relatively impervious to beam corrections, but rather representative of the beam-averaged physical conditions in the molecular gas.

Of the three galaxies in our sample with ${ }^{12} \mathrm{CO}(3-2)$ detections (Mrk 996 was observed but not detected, $R_{31}<0.7$ ), NGC 1140 shows the largest ratio $\left(R_{31}=2.00 \pm 0.45\right.$; with the exponential beam correction $\left.R_{31}=1.72 \pm 0.39\right)$, roughly equivalent to that found for NGC $3310\left(R_{31}=1.9 \pm 0.52\right)$ by Mao et al. (2010). Such a ratio exceeds typical values in luminous infrared galaxies (LIRGs) and those expected in the Orion "hot spots" (Papadopoulos et al. 2012). $R_{31}$ has been historically taken as an indicator of $\mathrm{H}_{2}$ density (e.g., Mauersberger et al. 1999), and high $R_{31}$ is possibly indicative of somewhat excited, optically thin, gas, although shocks and cool foreground absorbing layers are also possible causes (e.g., Oka et al. 2007). We shall explore this point further with physical radiative transfer models in Sect. 7.2.

\section{3. $\mathrm{Cl}(1-0) / \mathrm{CO}(1-0)$}

Together with $\mathrm{CO}$ and CII (at $158 \mu \mathrm{m}$ ), the atomic carbon line $[\mathrm{CI}](1-0)$ is an important coolant of the ISM (e.g., Bolatto et al. 1999; Gerin \& Phillips 2000; Bolatto et al. 2000a,b,c; Israel \& Baas 2002; Papadopoulos et al. 2004; Bayet et al. 2006; Israel 2009). Despite the difficulty of observation from the ground (the ${ }^{3} \mathrm{P}_{1}-{ }^{3} \mathrm{P}_{0}[\mathrm{CI}]$ transition lies at $\left.609 \mu \mathrm{m}\right)$, the potential of atomic carbon to trace molecular gas was recognized early on. Photo-Dissociation Regions (PDRs) are thought to be larger at low metallicity, because of the decreased attenuation of the UV field through the lower dust content (e.g., Lequeux et al. 1994; Bolatto et al. 1999). The brighter UV field in low $A_{V}$ environments tends to change the structure of PDRs, enlarging the region where atomic and ionized carbon dominate, 


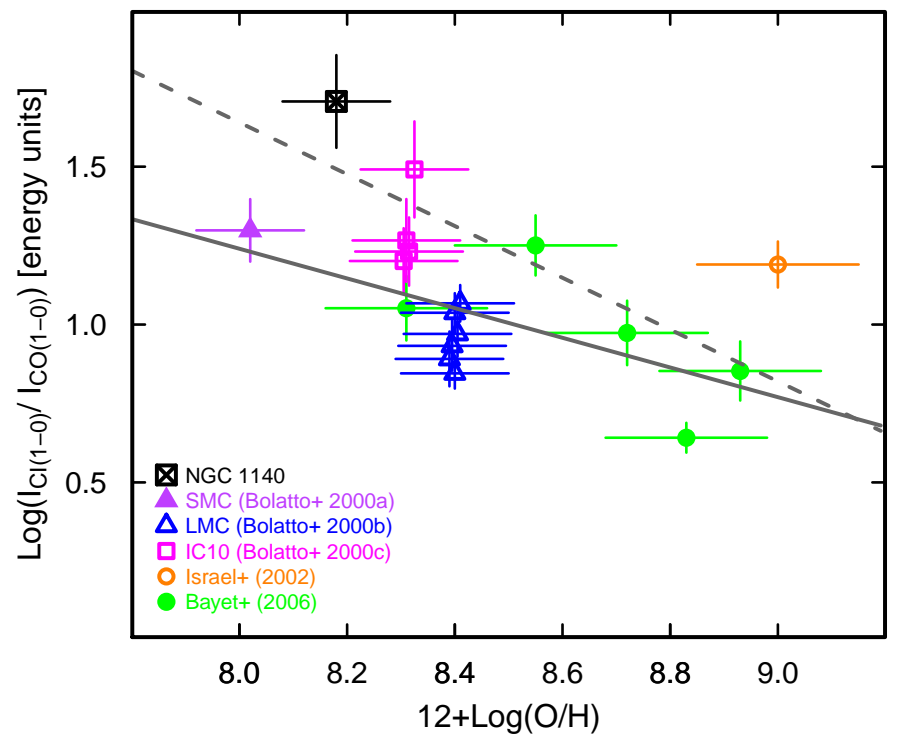

Fig. 5. Beam-corrected $[\mathrm{CI}] /{ }^{12} \mathrm{CO}(1-0)$ line ratios for $\mathrm{NGC} 1140$ and galaxies from the literature plotted against oxygen abundance $12+\log (\mathrm{O} / \mathrm{H})$. The solid line corresponds to the data regression by Bolatto et al. (2000a), and the dashed one to the PDR model by Bolatto et al. (1999). Beam dilution has been corrected for as described in Sect. 4.

and diminishing the region where $\mathrm{H}_{2}$ is associated with $\mathrm{CO}$ emission (e.g., Bolatto et al. 2013). The spatial distributions of $[\mathrm{CI}]$ and $\mathrm{CO}$ gas are similar in regions within the Milky Way (e.g., Kramer et al. 2004; Zhang et al. 2007), the Small Magellanic Cloud (SMC, Requena-Torres et al. 2016), and other galaxies (e.g., Israel \& Baas 2003; Krips et al. 2016), so [CI] seems to be tracing molecular gas. This is especially important in low-metallicity environments or cosmic-ray dominated regions where $\mathrm{CO}$ emission is suppressed (e.g., Hopkins et al. 2013; Bisbas et al. 2015, 2017), or in high-redshift galaxies where [CI] observations are facilitated (e.g., Walter et al. 2011).

We have obtained a $[\mathrm{CI}](1-0)$ measurement of one of our targets, NGC 1140, as shown in Fig. 5 where we plot the $[\mathrm{CI}] /{ }^{12} \mathrm{CO}(1-0)$ ratio (in energy units) together with similar measurements from the literature (Bolatto et al. 2000a,b,c; Israel \& Baas 2002; Bayet et al. 2006). We have applied the correction for beam dilution as described in Sect. 4 and Appendix A. The regression lines are taken from the PDR model by Bolatto et al. (1999, dashed line), and the best fit to their data (Bolatto et al. 2000a). Despite the paucity of data, the trend of enhanced $[\mathrm{CI}](1-0) /{ }^{12} \mathrm{CO}(1-0)$ with decreasing $\mathrm{O} / \mathrm{H}$ is evident.

The expected metallicity-dependent behavior of [CI](1-0) in PDR environments has been modeled by various groups (e.g., Papadopoulos et al. 2004) who confirm that the abundance of [CI] dominates that of $\mathrm{CO}$ for large regions within a low-metallicity cloud $\left(A_{V} \gtrsim 2\right)$. Glover \& Clark (2016) present more recent simulations of $[\mathrm{CI}](1-0)$ emission relative to ${ }^{12} \mathrm{CO}(1-0)$ as a function of metallicity. Our (beam-corrected) $[\mathrm{CI}](1-0) /{ }^{12} \mathrm{CO}(1-0)$ velocity-integrated temperature ratio for NGC 1140 of 0.65 (see Table 4) is more than a factor of two higher than their predictions for the highest PDR illumination they model $\left(G_{0}=100\right.$ in units of the interstellar radiation radiation field by Draine 1978), and the disagreement increases for less intense fields.

The $[\mathrm{CI}] /{ }^{12} \mathrm{CO}(1-0)$ ratio tends to decrease with increasing PDR density; the observed ratio for NGC 1140 is higher than the predictions of (solar metallicity) PDR models with volume number densities $n_{0} \sim 10^{4} \mathrm{~cm}^{-3}$ and $G_{0} \sim 10^{4}$ (Hollenbach et al. 1991). The observed $[\mathrm{CI}](1-0) /{ }^{13} \mathrm{CO}(2-1)$ for $\mathrm{NGC} 1140$ is a factor of 4-5 higher than the PDR predictions (for solar, and twice solar metallicities) by Meijerink et al. (2007), for volume number densities $n_{0} \gtrsim 10^{4} \mathrm{~cm}^{-3}$ and $G_{0} \sim 10^{5}$. However, they assume $\left[{ }^{12} \mathrm{CO} /{ }^{13} \mathrm{CO}\right]=40$; a lower isotopic ratio (see Sect. 7.3) or a lower metallicity would reduce the disagreement. In Sect. 7.3, we explore physical models of the gas in NGC 1140 and derive relative abundances of atomic carbon and $\mathrm{CO}$.

\section{Dense-gas tracers}

The relatively high critical densities of ${ }^{12} \mathrm{CO}(3-2)$ and $\mathrm{HCN}(1-$ $0)\left(n_{\text {crit }} \sim 10^{4}-10^{5} \mathrm{~cm}^{-3}\right)$ make these transitions good tracers of dense gas, which are possibly better correlated with SFR than the lowest- $J$ CO lines (Gao \& Solomon 2004; Narayanan et al. 2005, 2008; García-Burillo et al. 2012; Lada et al. 2012; Hopkins et al. 2013; Greve et al. 2014). Given the relatively high sSFRs of our targets $\left(s S F R \gtrsim 10^{-10} \mathrm{yr}^{-1}\right.$, see Paper I), here we examine luminosity correlations of ${ }^{12} \mathrm{CO}(3-2)$ and $\mathrm{HCN}(1-0)$ for these metal-poor galaxies and compare them with more massive metal-rich ones.

Figure 6 shows HCN luminosity plotted against FIR luminosity, $L_{\mathrm{FIR}}$ (left panel) and $\mathrm{CO}(1-0)$ luminosity, $L_{\mathrm{CO}(1-0)}^{\prime}$ (right). The only galaxy for which we have $\mathrm{HCN}(1-0)$ observations is NGC $1140^{4}$; also shown in Fig. 6 are luminous (and ultraluminous) infrared galaxies from Gao \& Solomon (2004), Graciá-Carpio et al. (2008), García-Burillo et al. (2012), together with regions within M 33 (Buchbender et al. 2013) and the Milky Way (MW, Wu et al. 2010); a luminous irregular galaxy, He 2-10, is also plotted (Santangelo et al. 2009). The dashed lines show a robust regression ${ }^{5}$ performed on all galaxies excepting He 2-10 and NGC 1140; the power-law index of the $L_{\mathrm{HCN}(1-0)}^{\prime}-L_{\mathrm{FIR}}$ correlation is $0.97 \pm 0.01$ and for $L_{\mathrm{HCN}(1-0)}^{\prime}{ }^{-}$ $L_{\mathrm{CO}(1-0)}^{\prime} 1.16 \pm 0.03$ (this last without the MW points, for which there were no $\mathrm{CO}(1-0)$ data). The slopes are consistent with previous work (e.g., Gao \& Solomon 2004; García-Burillo et al. 2012), and suggest that HCN in irregular galaxies such as $\mathrm{He} 2-$ 10 and NGC 1140 is somewhat deficient compared to $L_{\mathrm{FIR}}$ but within normal limits compared to $L_{\mathrm{CO}(1-0)}^{\prime}$.

Both He 2-10 and NGC 1140 contain extremely massive, compact star clusters (e.g., Johnson et al. 2000; de Grijs et al. 2004; Moll et al. 2007; super star clusters, SSCs), but He 210 is approximately solar metallicity (Kobulnicky et al. 1999) while NGC 1140 is $\sim 0.3 Z_{\odot}$. Feedback from these SSCs would be expected to substantially reduce the fraction of dense gas in these galaxies as traced by $\mathrm{HCN}(1-0)$ (Hopkins et al. 2013). Indeed, with $L_{\mathrm{CO}(1-0)}^{\prime} \sim 10^{8} \mathrm{~K} \mathrm{~km} \mathrm{~s}^{-1} \mathrm{pc}^{2}$, the $L_{\mathrm{HCN}(1-0)}^{\prime} / L_{\mathrm{CO}(1-0)}^{\prime}$ ratio for $\mathrm{He} 2-10$ is $\sim 0.02$, and $\lesssim 0.10$ for NGC 1140 (with $L_{\mathrm{CO}(1-0)}^{\prime} \sim 10^{6} \mathrm{~K} \mathrm{~km} \mathrm{~s}^{-1} \mathrm{pc}^{2}$ ), roughly consistent with the model predictions by Hopkins et al. (2013). More observations of HCN in such extreme galaxies are needed to further explore the survival of dense gas in these galaxies, and the ability of $\mathrm{HCN}$ to trace it (see also Sect. 7.3).

Figure 7 plots $L_{\mathrm{CO}(3-2)}^{\prime}$ vs. $L_{\mathrm{FIR}}$ and $L_{\mathrm{CO}(1-0)}^{\prime}$ as in Fig. 6 for $\mathrm{HCN}(1-0)$, but here for the four galaxies in our sample

\footnotetext{
4 We do not apply beam corrections to the upper limit, because of the similarity of the $\mathrm{HCN}(1-0)$ and ${ }^{12} \mathrm{CO}(1-0)$ beam sizes.

5 Here and throughout the paper we use the "robust" regression algorithm, effective for minimizing the effects of outliers, as implemented in $\mathrm{R}$, a free software environment for statistical computing and graphics, https://www.r-project.org/
} 


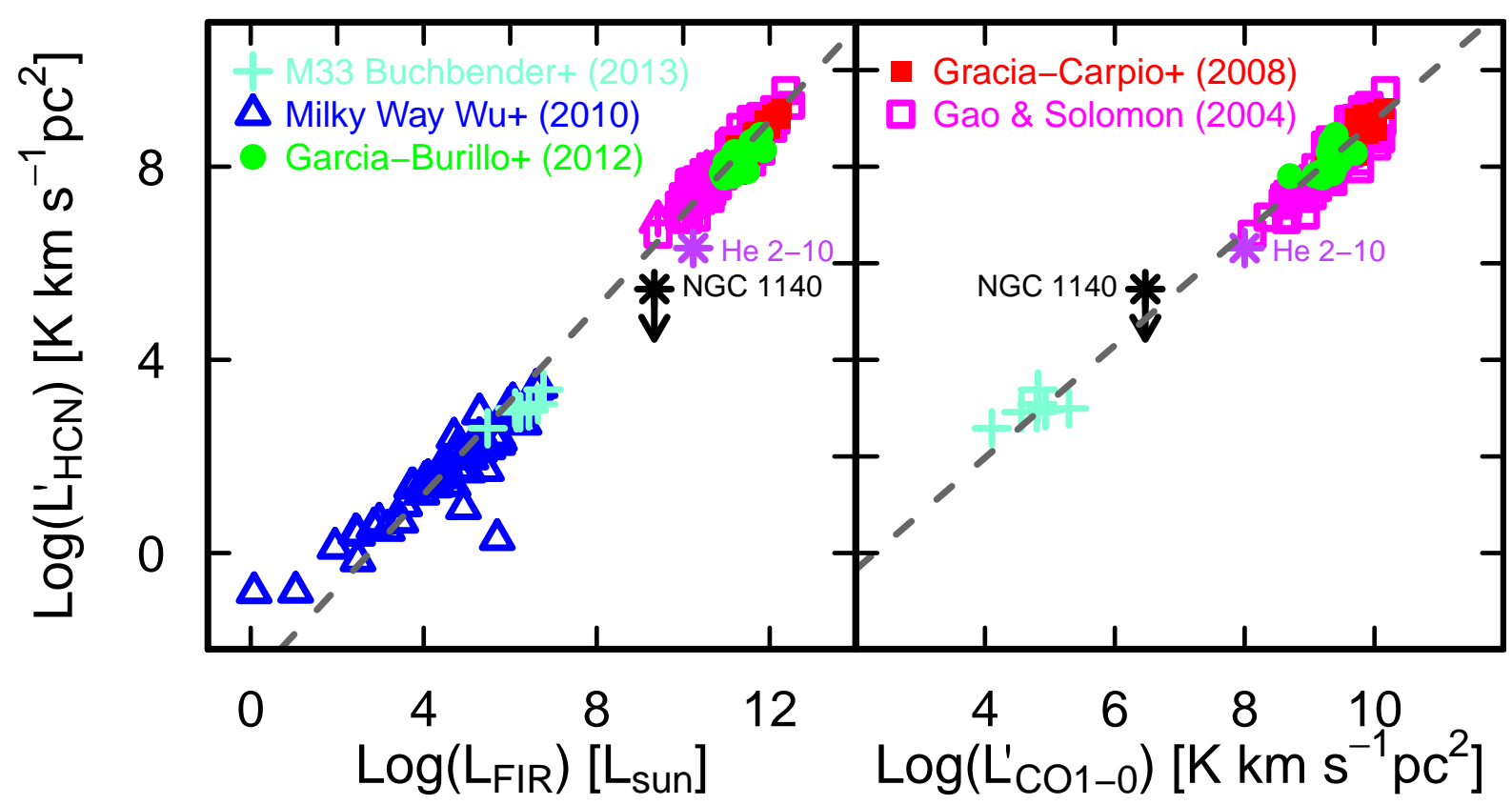

Fig. 6. HCN luminosity plotted against FIR luminosity, $L_{\mathrm{FIR}}$ (left panel) and $\mathrm{CO}(1-0)$ luminosity, $L_{\mathrm{CO}(1-0)}^{\prime}$ (right) for NGC 1140 . and other galaxies taken from the literature (see legend and text for more discussion). The dashed lines show a robust unweighted regression for $L_{\mathrm{HCN}(1-0)}^{\prime}$ against the two variables plotted along the $x$-axis, excluding He 2-10 and NGC 1140.

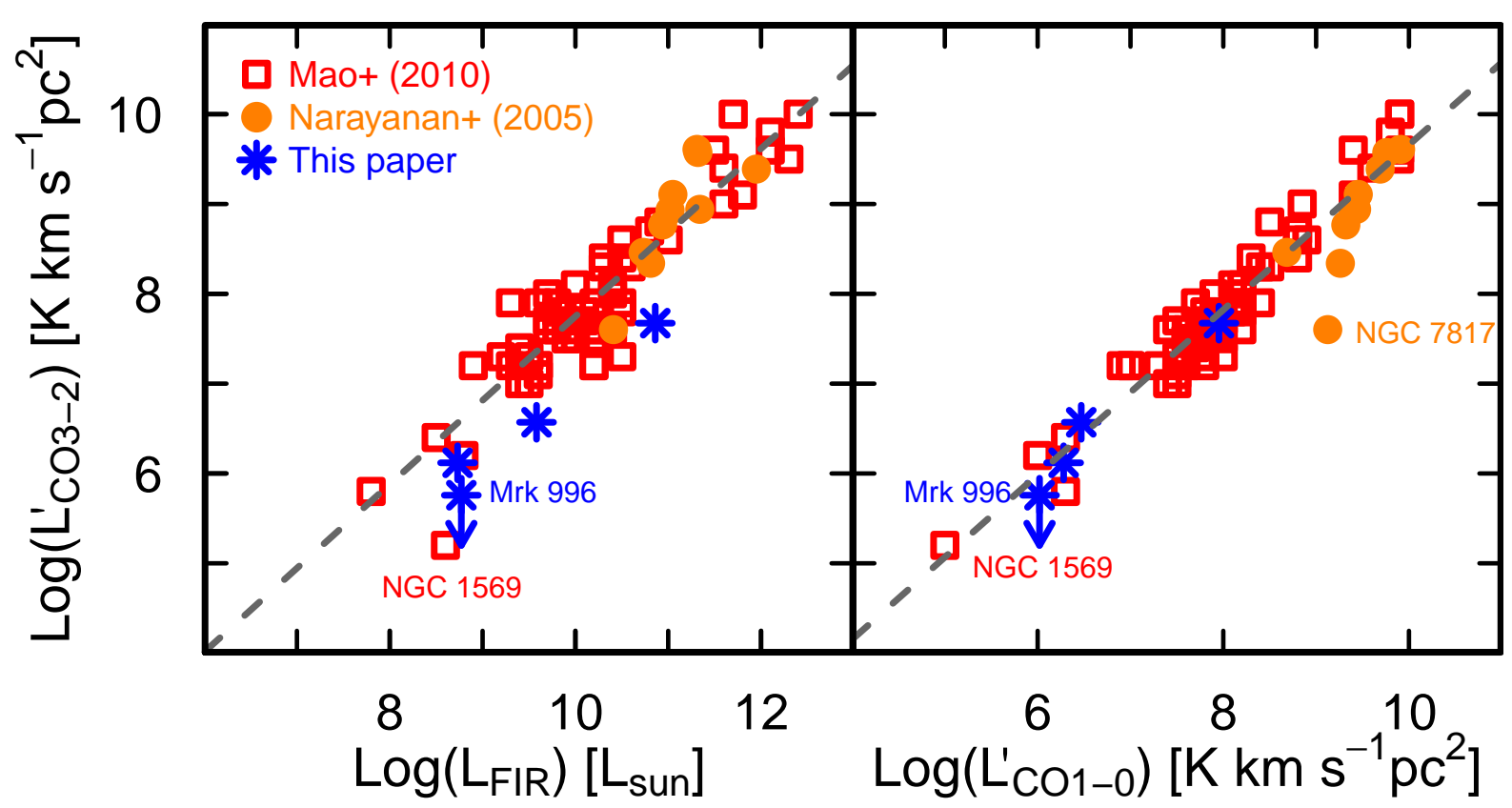

Fig. 7. $\mathrm{CO}(3-2)$ luminosity, $L_{\mathrm{CO}(3-2)}^{\prime}$, plotted against FIR luminosity, $L_{\mathrm{FIR}}$ (left panel) and $\mathrm{CO}(1-0)$ luminosity, $L_{\mathrm{CO}(1-0)}^{\prime}$ (right) for our targets. Also shown are values taken from Mao et al. (2010) and Narayanan et al. (2005), see text for more discussion. The dashed lines show a robust unweighted regression for $L_{\mathrm{CO}(3-2)}^{\prime}$ against the two variables plotted along the $x$-axis, not including our data. Some galaxies with extreme values are labeled. The left panel suggests that $\mathrm{CO}$ emission traced by the ${ }^{12} \mathrm{CO}(3-2)$ transition is deficient relative to $L_{\mathrm{FIR}}\left(\right.$ like ${ }^{12} \mathrm{CO}(1-0)$, see $\mathrm{Hunt}$ et al. $2015)$, but that ${ }^{12} \mathrm{CO}(3-2)$ relative to ${ }^{12} \mathrm{CO}(1-0)$ is roughly consistent, implying that the excitation conditions in these metal-poor galaxies are similar to those in the metal-rich galaxy samples taken from the literature.

which were observed in ${ }^{12} \mathrm{CO}(3-2)$ (Mrk 996, NGC 1140 , NGC 7077 UM 448). Like $\mathrm{HCN}(1-0),{ }^{12} \mathrm{CO}(3-2)$ is deficient relative to $L_{\mathrm{FIR}}$. The power-law indices of $0.93 \pm 0.05$ $\left(L_{\mathrm{CO}(3-2)}^{\prime}\right.$ vs. $\left.L_{\mathrm{FIR}}\right)$ and $0.96 \pm 0.04\left(L_{\mathrm{CO}(3-2)}^{\prime}\right.$ vs. $\left.L_{\mathrm{CO}(1-0)}^{\prime}\right)$ found by fitting the combination of the Mao et al. (2010) and Narayanan et al. (2005) samples are consistent with those found by Mao et al. (2010) and Greve et al. (2014), despite different fitting techniques for the latter; however the $L_{\mathrm{CO}(3-2)}^{\prime}$ vs. $L_{\mathrm{CO}(1-0)}^{\prime}$ 
slope is significantly flatter than the one of $\sim 1.5$ found by Narayanan et al. (2005) and used to calibrate the Hopkins et al. (2013) simulation results. The deficit of $L_{\mathrm{CO}(3-2)}^{\prime}$ relative to $L_{\mathrm{FIR}}$ is almost certainly due to metallicity as shown in Paper I; for metal-poor galaxies, we found an offset between the $\mathrm{CO}$ emission relative to the SFR in the sense that there is less $\mathrm{CO}$ for a given SFR value. This deficiency results from the metallicity dependence of the $\mathrm{CO}$ conversion factor to $\mathrm{H}_{2}$ mass. Interestingly, the four galaxies for which we have $\mathrm{CO}(3-2)$ measurements are not deficient in ${ }^{12} \mathrm{CO}(3-2)$ relative to ${ }^{12} \mathrm{CO}(1-0)$ in the other galaxies taken from the literature; they show similar CO luminosity ratios despite their low metallicity $\left(\sim 0.25 Z_{\odot}\right)$. This could be implying that excitations in these galaxies are similar to those in more metal-rich systems, a point which we explore more fully in the next section.

\section{Modeling physical conditions at low metallicity}

We now explore via modeling the physical conditions in the molecular gas in our metal-poor targets. Detailed physical modeling is possible only for NGC 1140 , for which we have four ${ }^{12} \mathrm{CO}$ and two ${ }^{13} \mathrm{CO}$ detections (see also Sect. 7.3). This is the first time that a low-metallicity galaxy outside the Local Group can be modeled in such detail with $\mathrm{CO}$; for the remaining galaxies we explore what can be learned from the available ${ }^{12} \mathrm{CO}$ detections.

\subsection{Local thermodynamic equilibrium}

For optically thin thermalized emission in Local Thermodynamic Equilibrium (LTE), antenna temperature is directly proportional to the column density in the upper level of the observed transition. With knowledge of the kinetic temperature, and under the above assumptions, we can estimate the column density of the species. Population (or rotation) diagrams are a useful tool for this (e.g., Goldsmith \& Langer 1999), through the analysis of the column density per statistical weight of different energy levels as a function of their energy above ground state. In LTE, the statistically-weighted column densities will be a Boltzmann distribution, so that the slope of a plot in natural logarithmic space will correspond to the inverse of the "rotational" temperature, equal to the kinetic temperature in the case of thermalized emission.

Despite its usefulness, there are several problems with such an approach. First, the assumptions required are not usually met by the physical conditions in molecular clouds; molecular emission in the ${ }^{12} \mathrm{CO}$ lines is usually optically thick and not necessarily thermalized. Second, to be able to correct the emission for the different beam sizes with which the various transitions are observed requires a knowledge of the source distribution and size. Nevertheless, a population diagram analysis sets useful limits on the column density and temperature and is a fruitful starting point for non-LTE considerations.

The column density of the upper energy state can be written as (e.g., Goldsmith \& Langer 1999):

$N_{\mathrm{u}}=\frac{8 \pi k v^{2} W}{h c^{3} A_{\mathrm{ul}}}\left(\frac{\Delta \Omega_{a}}{\Delta \Omega_{\mathrm{s}}}\right)\left(\frac{\tau}{1-\mathrm{e}^{-\tau}}\right)$,

where $W$ is the velocity-integrated main-beam brightness temperature, $A_{\mathrm{ul}}$ the Einstein spontaneous emission coefficient, and $\tau$ is the optical depth of the transition. For molecules in LTE, both a line's excitation temperature and a molecule's rotation

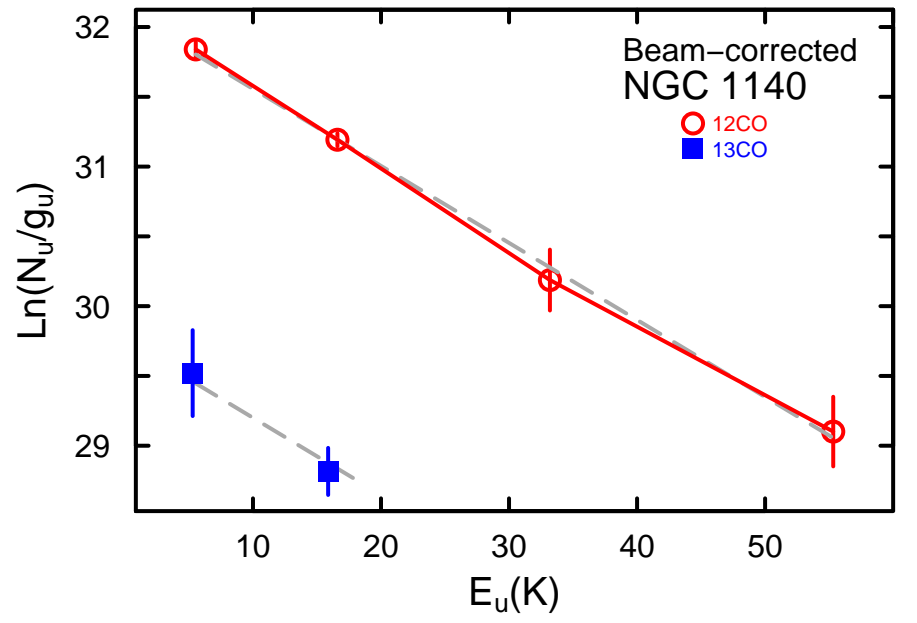

Fig. 8. Rotation or population level diagram for NGC 1140. A beam correction has been applied to the integrated intensities (see Sect. 4 for more details). Both ${ }^{12} \mathrm{CO}$ and ${ }^{13} \mathrm{CO}$ transitions are shown, as indicated by open (red) circles and filled (blue) squares, respectively. The gray dashed line for ${ }^{12} \mathrm{CO}$ corresponds to the best-fit regressions; the temperature for ${ }^{13} \mathrm{CO}$ is ill determined with only two transitions, so we fixed it to the ${ }^{12} \mathrm{CO}$ value of $T_{\mathrm{ex}}=18.1 \mathrm{~K}$.

temperature, which determines its relative energy level populations, equal the kinetic temperature $(\equiv T)$; the population in each level is then given by the Boltzmann distribution:

$N_{\mathrm{u}}^{\text {thin }}=\frac{N}{Z} g_{\mathrm{u}} \mathrm{e}^{-E_{\mathrm{u}} / k T}$,

where $N_{\mathrm{u}}^{\text {thin }}$ corresponds to optically-thin emission, ignoring the $\tau$ term in Eq. (1); $N$ is the total column density, $E_{\mathrm{u}}$ is the upper energy level, $g_{\mathrm{u}}$ is the statistical weight, and $Z$ is the partition function. Thus, under the assumption of LTE, and with knowledge of $T$, we can obtain the total molecular column density from the column density of any single transition:

$\ln \frac{N_{\mathrm{u}}^{\mathrm{thin}}}{g_{\mathrm{u}}}+\ln C_{\tau}=\ln N-\ln Z-\frac{E_{\mathrm{u}}}{k T}$,

where, following Goldsmith \& Langer (1999), we have equated the optical-depth correction factor $\tau /\left(1-\mathrm{e}^{-\tau}\right)$ to $C_{\tau}$, and $N_{\mathrm{u}}$ is calculated from the first two factors in Eq. (1). To evaluate the population diagrams numerically, the transition frequencies, upper energy levels, Einstein spontaneous emission, and statistical weights $\left(v, E_{\mathrm{u}}, A_{\mathrm{ul}}, g_{\mathrm{u}}\right)$ for the ${ }^{12} \mathrm{CO}$ and ${ }^{13} \mathrm{CO}$ molecules were calculated using physical quantities from Schöier et al. (2005) and found to be highly consistent with the data from HITRAN (Rothman et al. 2009).

We first assume that the emission is optically thin, with $\tau \ll 1\left(C_{\tau}=1\right)$. A CO population diagram with this assumption for NGC 1140 is shown in Fig. 8, including the two available ${ }^{13} \mathrm{CO}$ transitions. Here, as elsewhere, we have used the velocityintegrated $T_{\mathrm{mb}}$ corrected to a $22^{\prime \prime}$ beam size as described in Sect. 4 and Appendix A. By fitting the $\ln \left(N_{\mathrm{u}} / g_{\mathrm{u}}\right)$ vs. $E_{\mathrm{u}}$ values with a linear regression (shown as a gray dashed line), we were able to infer the total ${ }^{12} \mathrm{CO}$ and ${ }^{13} \mathrm{CO}$ column densities and temperatures: $T_{12 \mathrm{CO}}=18.1 \pm 1.0 \mathrm{~K} ; \log \left(N_{12 \mathrm{CO}}\right)=14.7 \mathrm{~cm}^{-2}$; $T_{13 \mathrm{CO}}=15 \mathrm{~K}$. With only two transitions, the ${ }^{13} \mathrm{CO}$ temperature is ill determined, so we fixed it to $T_{12 \mathrm{CO}}$ and obtained $\log \left(N_{13 \mathrm{CO}}\right)=13.6 \mathrm{~cm}^{-2}$.

We then attempted to correct for optical depth effects, according to Goldsmith \& Langer (1999, their Eq. (27)): the 


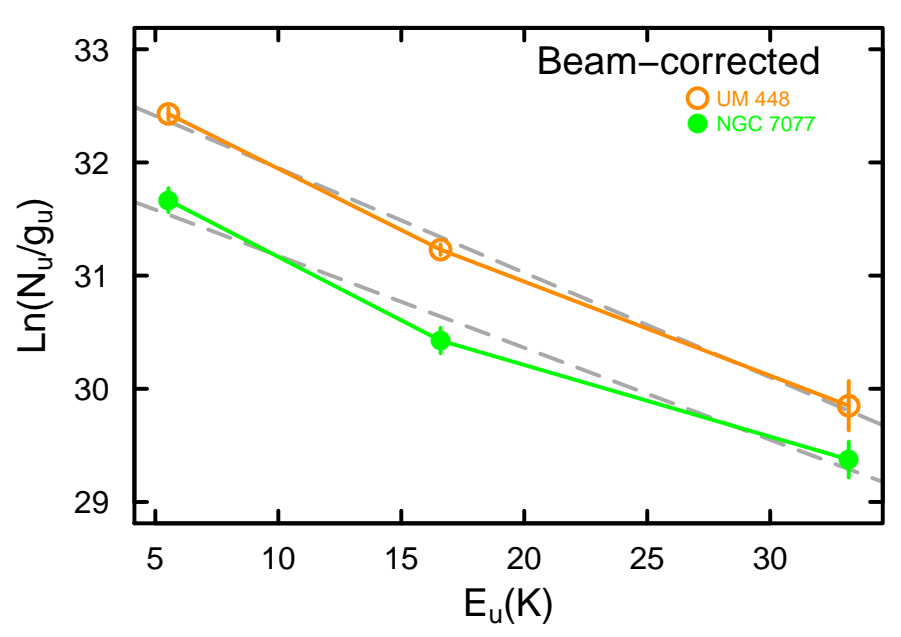

Fig. 9. Rotation or population level diagram for UM 448 and NGC 7077. A beam correction has been applied to the integrated intensities (see Sect. 4 for more details). The gray dashed line corresponds to the bestfit regression; as shown by the legend, the galaxies are differentiated by different symbols for ${ }^{12} \mathrm{CO}$ emission (open (orange) circles and filled (green) circles, for UM 448 and NGC 7077, respectively).

largest correction term $C_{\tau}$ is for ${ }^{12} \mathrm{CO}(4-3)$ with $C_{\tau}=1.01$, and the largest optical depth $\tau \sim 0.002$. If we take these results at face value, and infer the ${ }^{12} \mathrm{CO} /{ }^{13} \mathrm{CO}$ abundances by taking the ratio of the total column densities, we obtain a value of ${ }^{12} \mathrm{CO} /{ }^{13} \mathrm{CO} \sim 12-13$. To our knowledge this is one of the lowest values ever inferred for a galaxy, but cannot be considered reliable because of the assumption of optically thin gas in the LTE analysis. We will explore this further in Sects. 7.3 and 8 .

The population diagrams under the optical-thin assumption for UM 448 and NGC 7077 (the only other galaxies for which we have ${ }^{12} \mathrm{CO}(3-2)$ detections) are shown in Fig. 9. The observations were corrected for beam dilution as described in Sect. 4 and Appendix A. Again, from the best-fit regression, we infer $T$ and total $N_{\mathrm{CO}}$ column densities: $T_{12 \mathrm{CO}}=10.8 \pm 0.8 \mathrm{~K}$ and $\log \left(N_{12 \mathrm{CO}}\right)=15.0 \mathrm{~cm}^{-2}$ for UM 448 ; and $T_{12 \mathrm{CO}}=12.3 \pm 2.0 \mathrm{~K}$ and $\log \left(N_{12 \mathrm{CO}}\right)=14.7 \mathrm{~cm}^{-2}$ for NGC 7077. As for NGC 1140, we inferred the optical depths for the various transitions, and for both galaxies find that the largest $\tau$ is for the $J=2-1$ transition with $\tau_{\mathrm{CO}}=0.001$ (NGC 7077) and $\tau_{\mathrm{CO}}=0.002$ (UM 448).

\subsection{Modeling ${ }^{12} \mathrm{CO}$ line ratios with $\mathrm{RADEX}$}

To better understand the observed ${ }^{12} \mathrm{CO}$ line ratios in the galaxies in our sample, in particular the high ratio of NGC 1140 compared with other, more metal-rich, galaxies (see Figs. 3, 4), we have plotted the line ratios predicted by RADEX as a function of $T_{\text {kin }}$ in Fig. $10\left(R_{21}\right.$ and $R_{31}$ for NGC 1140 have been corrected for beam dilution as described in Sect. 4 and Appendix A). Two sets of column densities $N_{12 \mathrm{CO}}$ and volume densities $n_{\mathrm{H} 2}$ are illustrated in the figure; the solid (purple, upper) line passing through the locus of NGC 1140 corresponds to the approximate best-fit RADEX model described in Sect. 7.3.

It is evident from Fig. 10 that the combination of moderately low column density (and thus low $\tau_{\mathrm{CO}}$ ) and high $n_{\mathrm{H} 2}$ is a necessary ingredient for the high line ratios observed in NGC 1140. Line ratios would be high also in the case of high $N_{12 \mathrm{CO}}$ and high $n_{\mathrm{H} 2}$ (see lower blue solid line), but only at high temperatures; this is the case in NGC 1068 where gas is warm and dense in the circumnuclear disk around the active nucleus (Viti et al. 2014).
We discuss below in Sect. 7.3.3 why we consider high temperatures in NGC 1140 to be unlikely. In the case of more moderate $n_{\mathrm{H} 2}$ (here illustrated as $10^{4} \mathrm{~cm}^{-3}$ ), there is no $T_{\text {kin }}$ that can sufficiently excite the upper $J$ level.

The remaining galaxies for which we have ${ }^{12} \mathrm{CO}(3-2)$ observations (Mrk 996, NGC 7077, UM 448) are also shown in the right panel of Fig. 10. The $R_{31}$ values of these are less extreme, and can be well modeled with $\mathrm{H}_{2}$ volume densities as low as $10^{4} \mathrm{~cm}^{-3}$. In these galaxies, the gas does not necessarily have to be cool and optically thin (i.e., low $N_{12 \mathrm{CO}}$ ) in order to achieve the observed $R_{31}$; it can be warmer and have a higher column density than in NGC 1140.

\subsection{RADEX models for NGC 1140}

To determine the excitation conditions of the molecular gas in NGC 1140, we have fitted the six ${ }^{12} \mathrm{CO}$ and ${ }^{13} \mathrm{CO}$ detected transitions with the radiative transfer code RADEX developed by van der Tak et al. (2007), using the uniform sphere approximation to calculate the escape probabilities. A one-zone model was adopted, namely the kinetic temperature and $\mathrm{H}_{2}$ volume density were required to be the same for all molecules, implying that all transitions sample the same gas. We calculated RADEX predictions for $\Delta V=10$ and $20 \mathrm{~km} \mathrm{~s}^{-1}$, covering the following range of parameters sampled in logarithmic steps ${ }^{6}$ :

- $\mathrm{H}_{2}$ volume densities $n_{\mathrm{H} 2}=10^{2}-10^{8} \mathrm{~cm}^{-3}$;

- kinetic temperature $T_{\text {kin }}=5-200 \mathrm{~K}$;

- CO column densities $N_{\mathrm{CO}}=10^{12}-10^{20} \mathrm{~cm}^{-2}$.

All parameters were calculated assuming a cosmic microwave background radiation temperature of $T=2.73 \mathrm{~K}$. The ${ }^{13} \mathrm{CO}$ abundance was included in the calculation through a second RADEX grid for the isotope; the column density $N_{13 C O}$ was adjusted by sampling a range of abundances (from $N_{12 \mathrm{CO}} / N_{13 \mathrm{CO}}=1$ to 100), and refining the fineness of the grid once the initial bestfit parameters were established. Ultimately, $\gtrsim 400000$ total models in the combined ${ }^{12} \mathrm{CO}+{ }^{13} \mathrm{CO}$ grid were sampled.

The observed emission is generally only a small fraction of integrated line intensity from RADEX (and Large-Velocity Gradient, LVG) models. The fraction of these two quantities, $F=\left(\Delta v_{\text {obs }} T_{\text {obs }}\right) /\left(\Delta V_{\text {model }} T_{\text {model }}\right)$, is the filling factor. We incorporated $F$ as a free parameter in the models, and fit the grid of RADEX models via a $\chi^{2}$ minimization technique (e.g., Nikolić et al. 2007):

$\chi^{2}=\sum_{i=1}^{6}\left(\frac{F I_{i}^{\mathrm{mod}}-I_{i}^{\mathrm{obs}}}{\Delta I_{i}^{\mathrm{obs}}}\right)^{2}$

where $I_{i}^{\mathrm{obs}}$ is the velocity-integrated $T_{\mathrm{mb}}$ of the $i$ th transition, $I_{i}^{\mathrm{mod}}$ the $i$ th model prediction, and $\Delta I_{i}^{\mathrm{obs}}$ are the $1 \sigma$ uncertainties given in Table 4.

We also experimented with a minimization technique not including the filling factor $F$, but rather based on line ratios:

$\chi^{2}=\sum_{j=1}^{5}\left(\frac{R_{j}^{\mathrm{mod}}-R_{j}^{\mathrm{obs}}}{\Delta R_{j}^{\mathrm{obs}}}\right)^{2}$

where $R_{j}^{\mathrm{obs}}$ is the $j$ th velocity-integrated temperature ratio, $R_{j}^{\bmod }$ is the corresponding model line ratio, and $\Delta R_{j}^{\text {obs }}$ are the $1 \sigma$ uncertainties. These fits do not explicitly require equality of the

6 Temperature was sampled linearly. 

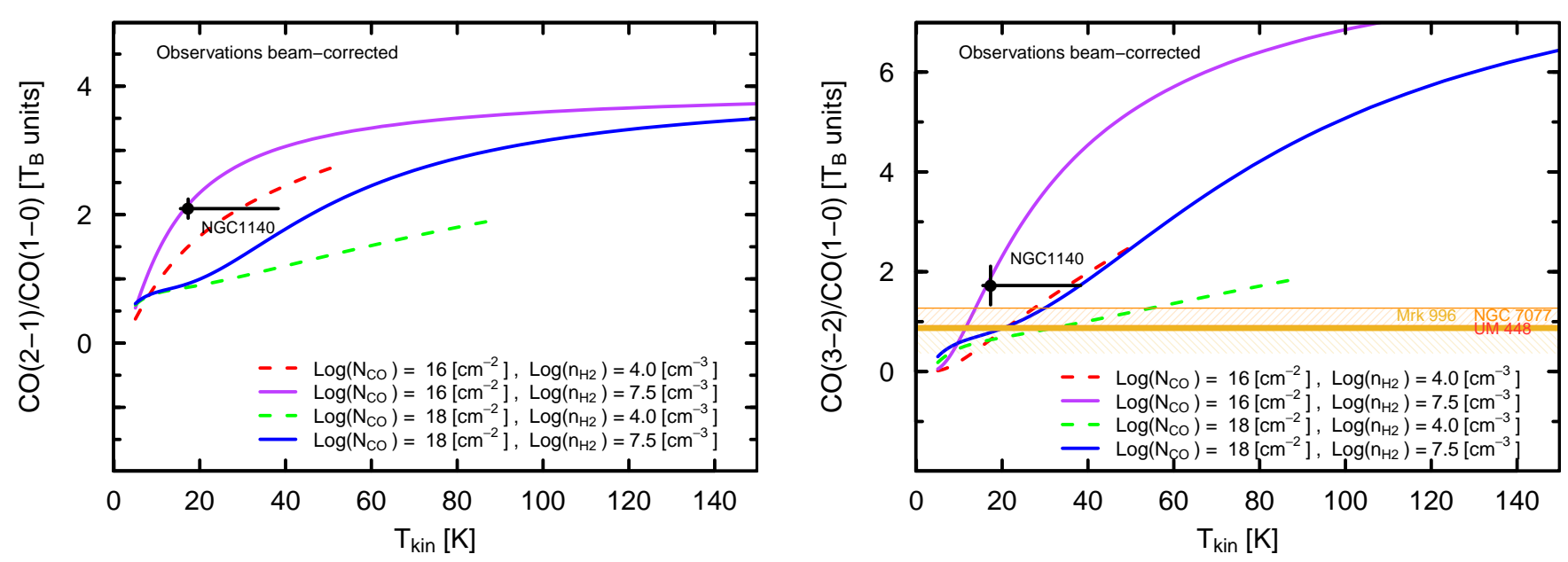

Fig. 10. RADEX models (with $\Delta V=20 \mathrm{~km} \mathrm{~s}^{-1}$ ) of ${ }^{12} \mathrm{CO}$ line ratios plotted against $T_{\text {kin }}$ : the left panel shows ${ }^{12} \mathrm{CO}(2-1) /{ }^{12} \mathrm{CO}(1-0)$ and the right ${ }^{12} \mathrm{CO}(3-2) /{ }^{12} \mathrm{CO}(1-0)$. The observations of NGC 1140, shown as a (black) filled circle, have been corrected for beam dilution assuming an exponential source distribution as described in Sect. 4 (see also Figs. 3 and 4). The approximate best-fit RADEX model for NGC 1140 (see Sect. 7.3) is shown in both panels as (purple) solid curves that coincide very well with the observations of NGC 1140. Also shown are three additional parameter combinations of ${ }^{12} \mathrm{CO}$ column density $N_{12 \mathrm{CO}}$ and volume density $n_{\mathrm{H} 2}$. The $R_{31}$ parameter space occupied by the other galaxies (like Fig. 4, no beam corrections) is shown in the right-hand panel as a hatched region and solid horizontal lines (NGC 7077, UM 448, and an upper limit for Mrk 996).

filling factor $F$ and thus may be more accommodating. We experimented with several sets of line ratios, in order to avoid potentially biasing the fits to any of the individual ratios.

As described in Sect. 4, we explored two different methods for correcting the velocity-integrated $T_{\mathrm{mb}}$ (and uncertainties) to a $22^{\prime \prime}$ beam size: (1) analyzing the cool-dust distribution and assuming that the molecular gas is distributed like the dust (see Appendix A); and (2) assuming the molecular gas is distributed in a Gaussian, and letting the Gaussian's FWHM $\left(\theta_{\mathrm{s}}\right)$ be a free parameter in the fit (see Appendix B). We used both methods to calculate the observed $I_{i}^{\text {obs }}$ in Eqs. (4), (5). In the first case based on the fitted exponential function of the dust emission, $I_{i}^{\text {obs }}$ were calculated independently of the fitting procedure. Thus for the six intensities (Eq. (4)), we fit five parameters $\left(n_{\mathrm{H} 2}, T_{\mathrm{kin}}\right.$, $\left.N_{\mathrm{CO}}\left({ }^{12} \mathrm{CO}\right),\left[{ }^{12} \mathrm{CO}\right] /\left[{ }^{13} \mathrm{CO}\right], F\right)$, and for the five independent line ratios (Eq. (5)), we fit four parameters (same as before, but not including $F$ ). For the second case of Gaussian beam corrections, $I_{i}^{\text {obs }}$ were calculated within the fit procedure; we explored $\theta_{\mathrm{s}}$ ranging from $2^{\prime \prime}$ to $40^{\prime \prime}$, and narrowed the step size in successive iterations. Thus for the Gaussian beam corrections, the fitting procedure comprised an additional parameter, $\theta_{\mathrm{s}}$, and the minimum $\chi^{2}$ value includes this dependence. All the $\chi^{2}$ values quoted here are not reduced, but rather the total summed $\chi^{2}$.

Table 6 gives the best-fit parameters obtained for NGC 1140. The different fitting methods and even the different beam correction approaches give very similar results, namely cool temperatures, high volume densities, low $\mathrm{CO}$ opacities, and low ${ }^{12} \mathrm{CO} /{ }^{13} \mathrm{CO}$ abundance ratios. The main difference among the fits is the CO column density, $N_{12 \mathrm{CO}}$, which for a given $\Delta V$ varies by a factor of $\sim 4$. Because the opacity in the RADEX models $\tau \propto N_{\text {co }} / \Delta V$, and $\tau$ governs the line ratios and thus must remain relatively constant, for higher $\Delta V, N_{12 \mathrm{CO}}$ is consequently higher within the resolution of our RADEX grid. For higher $N_{12 \mathrm{CO}}$, the filling factor $F$ is also commensurately lower, which we will discuss in Sect. 7.3.1. In any case, the low values of $\chi^{2}$ imply that a one-zone spherical cloud with uniform physical properties gives a good fit to the molecular gas emission in NGC 1140. The extremely low ${ }^{12} \mathrm{CO} /{ }^{13} \mathrm{CO}$ abundance ratio of $\sim 8-20$ is obtained for NGC 1140 even with a non-LTE analysis; we discuss this unexpected result further in Sect. 8.1.

Figure 11 shows the $\chi^{2}$ surface for the Set 1 line ratios ${ }^{7}$ with the exponential beam correction as a function of the fitted parameters ( $T_{\text {kin }}$ vs. $n_{\mathrm{H} 2}$ in the left panel, and $N_{12 \mathrm{CO}}$ vs. $\tau\left[{ }^{12} \mathrm{CO}(1-0)\right]$ in the right). In both panels, the error bars correspond to $\chi^{2} \lesssim 1$ at the best-fit value, rather than the full posterior range given in Table 6. It can also be seen from Fig. 11 that the parameters are fairly well constrained by the model; specifically, the volume density $n_{\mathrm{H} 2}$ cannot be much lower than $\sim 10^{5} \mathrm{~cm}^{-3}$, and because of the well-known degeneracy between $T_{\text {kin }}$ and $n_{\mathrm{H} 2}$, the temperature also cannot be much greater than $\sim 20 \mathrm{~K}$.

Figure 12 gives the $\chi^{2}$ surface for the Set 1 line ratios using the Gaussian beam correction plotted against the ${ }^{12} \mathrm{CO}$ column density $N_{12 \mathrm{CO}}$ and the Gaussian FWHM $\theta_{\mathrm{s}}$. The fit is somewhat sensitive to the assumed $\theta_{\mathrm{s}}$; for $\theta_{\mathrm{s}} \lesssim 15^{\prime \prime}$ and for $\theta_{\mathrm{s}} \gtrsim 34^{\prime \prime}$, $\chi^{2}>10$, and thus is not plotted. The best-fit Gaussian sizes range from $26^{\prime \prime}$ to $31^{\prime \prime}$, implying that under the assumption of a Gaussian distribution, the macroscopic size of the emission is $\gtrsim 2 \mathrm{kpc}$. This would imply that the $\mathrm{CO}$ emission is more extended than the dust $\left(F W H M\left(r_{\mathrm{s}}\right) \sim 850 \mathrm{pc}\right.$, see Appendix A), which seems unlikely. In any case, even the largest CO beam is sampling less than half of the galaxy ISM; as shown in Fig. A.1, 50\% of the dust emission lies within an effective radius (diameter) of $\sim 20^{\prime \prime}$ $\left(40^{\prime \prime}, 3.8 \mathrm{kpc}\right)$.

It should be emphasized that small $\chi^{2}$ values are not easy to achieve, even with several trials with many different formulations of beam corrections, in addition to the ones described in Sect. 4 and in Appendices A and B. The small $\chi^{2}$ values of the fits in Table 6 are telling us that for reasonable assumptions for beam corrections, the single-zone RADEX models can well accommodate the data. Moreover, the physical conditions inferred from the different fitting techniques are mutually consistent, independently of the kind of beam correction. Because of this similarity, in what follows, we will use the exponential

\footnotetext{
7 "Set 1" was taken with the ratios relative to the adjacent lower$J$ line, specifically: $\left[{ }^{12} \mathrm{CO}(2-1) /{ }^{12} \mathrm{CO}(1-0),{ }^{12} \mathrm{CO}(3-2) /{ }^{12} \mathrm{CO}(2-1)\right.$, $\left.{ }^{12} \mathrm{CO}(4-3) /{ }^{12} \mathrm{CO}(3-2),{ }^{13} \mathrm{CO}(1-0) /{ }^{12} \mathrm{CO}(1-0),{ }^{13} \mathrm{CO}(2-1) /{ }^{13} \mathrm{CO}(1-0)\right]$.
} See Table 6 for more details. 

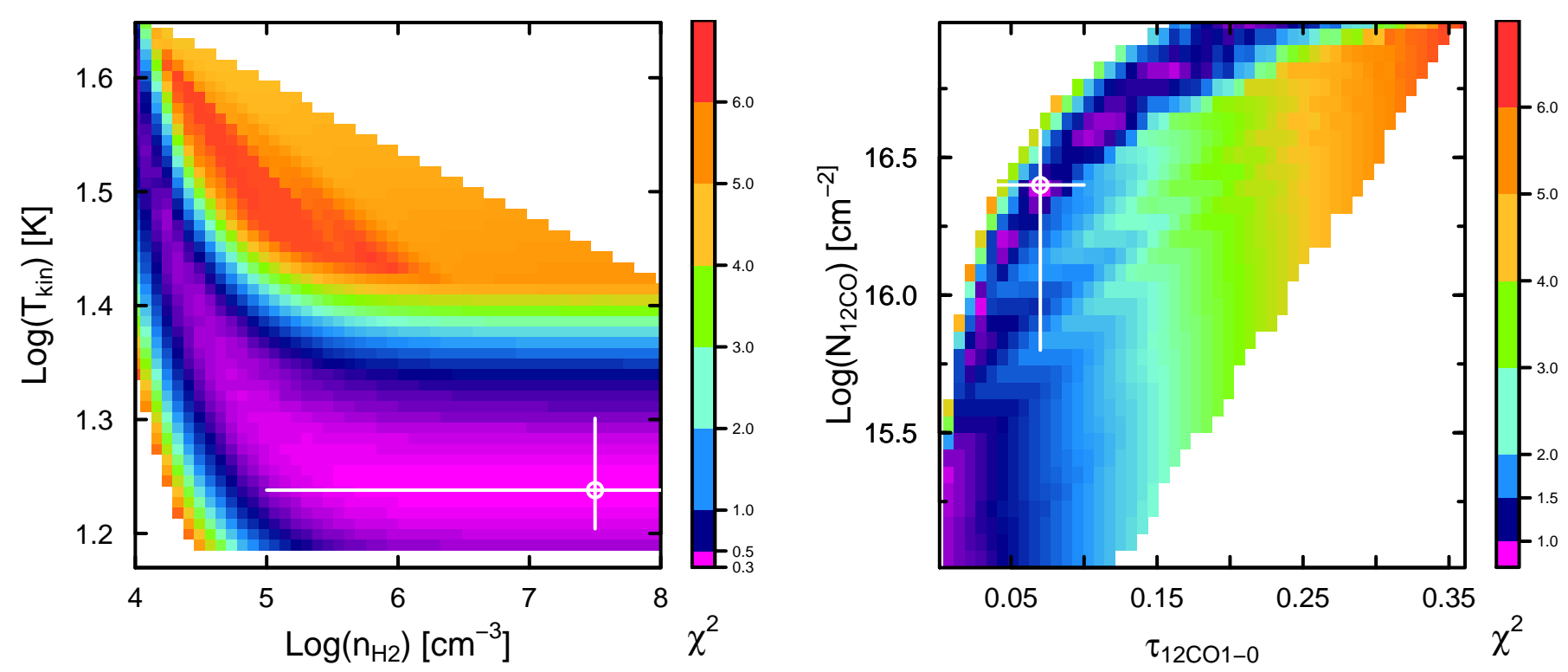

Fig. 11. RADEX fit $\chi^{2}$ surface (for the "Set 1" line ratios) with the exponential beam correction as a function of $T_{\mathrm{kin}}$ and $n_{\mathrm{H} 2}$ in the left panel, and as a function of $N_{12 \mathrm{CO}}$ and $\tau\left({ }^{12} \mathrm{CO}(1-0)\right)$ in the right. The RADEX models shown here have $\Delta V=20 \mathrm{~km} \mathrm{~s}^{-1}$. The associated $\chi^{2}$ values are shown as a side-bar color table. The $\chi^{2}$ color table in the right panel is different from the left because the interpolation used for the graphic display suffers from the coarser resolution in column density. The best-fit values are shown as an open (white) circle; the error bars shown consider a slightly more limited range in $\chi^{2}$ respect to Table 6.

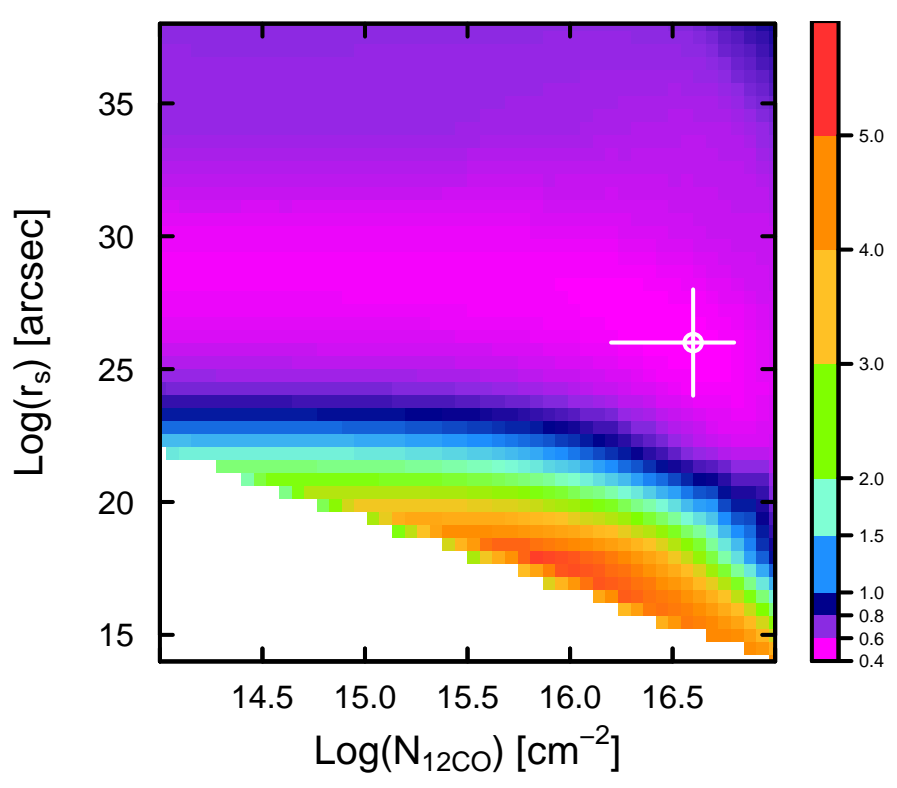

Fig. 12. RADEX fit $\chi^{2}$ surface (for the "Set 1 " line ratios, assuming the Gaussian-derived beam correction factors) as a function of $N_{12 \mathrm{CO}}$ and Gaussian FWHM $\theta_{\mathrm{s}}$ in the left panel, The RADEX models shown here have $\Delta V=20 \mathrm{~km} \mathrm{~s}^{-1}$; the associated $\chi^{2}$ values are shown as a side-bar color table. The best-fit values are shown as an open (white) circle; the error bars shown consider a slightly more limited range in $\chi^{2}$ respect to Table 6.

beam corrections described in Appendix A; the virtue of these is that they can be measured or estimated for all the galaxies observed, under the assumption that on kpc scales the molecular gas is following the dust.

\subsubsection{Filling factor}

The mean ${ }^{12} \mathrm{CO}$ line width of the various components in NGC 1140 (see Table 4) is $\sim 21 \mathrm{~km} \mathrm{~s}^{-1}$; we thus prefer the
RADEX fits with $\Delta V=20 \mathrm{~km} \mathrm{~s}^{-1}$. For $\Delta V=20 \mathrm{~km} \mathrm{~s}^{-1}$, fitting the intensities as in Eq. (4) gives a best-fit filling factor $F$ of $0.10 \pm 0.06$. This best-fit $F$ is rather large, but related to the somewhat low $N_{12 \mathrm{CO}}$ value of $10^{15.8} \mathrm{~cm}^{-2}$; as discussed below, the fits of the line ratios as in Eq. (5) give higher $N_{12 \mathrm{CO}}$ thus consequently lower, possibly more realistic, values of $F$.

For the line-ratio fits, the best-fit RADEX model for NGC 1140 predicts a velocity-integrated brightness temperature $T_{B}\left({ }^{12} \mathrm{CO}(1-0)\right)=21.0 \mathrm{~K} \mathrm{~km} \mathrm{~s}^{-1}$; from Table 4 , we have a beamcorrected velocity-integrated $T_{\mathrm{mb}}$ of $0.56 \mathrm{~K} \mathrm{~km} \mathrm{~s}^{-1}$. Dividing the two values gives a nominal filling factor of $\sim 0.03$. Similar filling factors are derived for the other transitions including ${ }^{13} \mathrm{CO}$, as a consequence of the assumption of a single-zone model. If we compare with the model from the "other" $\Delta V=20 \mathrm{~km} \mathrm{~s}^{-1}$ lineratio fits (see Table 7.3), with lower $N_{12 \mathrm{CO}}$ and $\tau$ (see Table 6), we obtain a slightly higher value, $F \sim 0.04$. Using the mainbeam peak temperatures rather than velocity-integrated values (see Table 4), and adjusting to the common $22^{\prime \prime}$ beam size, gives filling factors of $\sim 0.01$.

These filling factors of a few percent for the molecular gas in NGC 1140 are similar to those found from high-resolution ALMA maps of 30 Doradus in the Large Magellanic Cloud (LMC, Indebetouw et al. 2013). They are also similar to those obtained by a similar $\chi^{2}$ analysis of non-LTE models of singledish line ratios in the SMC and the LMC (Nikolić et al. 2007); these last are as high as $\sim 0.14$ (for what they consider to be good fits with $\chi^{2} \lesssim 2$ ), consistent with $F=0.1$ for NGC 1140 obtained from the intensity fitting.

\subsubsection{CO excitation ladder}

The modeled CO excitation ladders for NGC 1140 are shown in Fig. 13. As in Fig. 10, the observations have been corrected for beam size as described in Sect. 4 and Appendix A. The line segments show the best-fit RADEX model for both ${ }^{12} \mathrm{CO}$ and ${ }^{13} \mathrm{CO}$ transitions adopting the best fit for the Set 1 line ratios with $\Delta V=20 \mathrm{~km} \mathrm{~s}^{-1}$; the blue area corresponds to the range of ratios 
Table 6. Best-fit RADEX parameters for NGC 1140.

\begin{tabular}{|c|c|c|c|c|c|c|c|c|c|c|c|}
\hline $\begin{array}{c}\Delta V \\
\left(\mathrm{~km} \mathrm{~s}^{-1}\right) \\
(1)\end{array}$ & $\begin{array}{l}\chi^{2} \\
(2) \\
\end{array}$ & $\begin{array}{c}{ }^{12} \mathrm{CO}(1-0) \\
(3)\end{array}$ & $\begin{array}{c}{ }^{12} \mathrm{CO}(2-1) \\
\text { (4) }\end{array}$ & $\begin{array}{c}{ }^{12} \mathrm{CO}(3-2) \\
(5)\end{array}$ & $\begin{array}{l}\tau \\
{ }^{12} \mathrm{CO}(4-3) \\
\quad(6)\end{array}$ & $\begin{array}{c}{ }^{13} \mathrm{CO}(1-0) \\
(7)\end{array}$ & $\begin{array}{c}{ }^{13} \mathrm{CO}(2-1) \\
(8)\end{array}$ & $\begin{array}{l}T_{\text {kin }} \\
(\mathrm{K}) \\
(9) \\
\end{array}$ & $\begin{array}{c}\log \left(n_{\mathrm{H} 2}\right) \\
\left(\mathrm{cm}^{-3}\right) \\
(10)\end{array}$ & $\begin{array}{c}\log \left(N_{122 \mathrm{CO}}\right) \\
\left(\mathrm{cm}^{-2}\right) \\
(11)\end{array}$ & $\begin{array}{c}{ }^{12} \mathrm{CO} /{ }^{13} \mathrm{CO} \\
(12)\end{array}$ \\
\hline \multicolumn{12}{|c|}{ Fit to intensities with exponential beam correction } \\
\hline 10 & 0.94 & 0.02 & 0.06 & 0.06 & 0.04 & 0.002 & 0.006 & 17.3 & 7.4 & 15.6 & 8 \\
\hline- & $0.94-1.93$ & $0.02-0.04$ & $0.05-0.10$ & $0.06-0.10$ & $0.04-0.06$ & $0.002-0.004$ & $0.005-0.010$ & $16.1-18.7$ & $4.9-8.0$ & $15.6-15.8$ & $8-10$ \\
\hline 20 & 0.93 & 0.02 & 0.05 & 0.05 & 0.03 & 0.002 & 0.005 & 16.7 & 7.5 & 15.8 & 8 \\
\hline- & $0.93-1.93$ & $0.01-0.05$ & $0.03-0.13$ & $0.03-0.13$ & $0.02-0.08$ & $0.001-0.005$ & $0.003-0.012$ & $16.1-19.4$ & $4.9-8.0$ & $15.6-16.2$ & $8-10$ \\
\hline \multicolumn{12}{|c|}{ Fit to intensities with Gaussian beam correction ${ }^{a}$} \\
\hline 10 & 0.95 & 0.02 & 0.06 & 0.06 & 0.04 & 0.002 & 0.005 & 18.0 & 7.1 & 15.6 & 10 \\
\hline - & $0.95-1.95$ & $0.01-0.06$ & $0.05-0.15$ & $0.06-0.16$ & $0.04-0.10$ & $0.001-0.005$ & $0.004-0.014$ & $16.1-26.3$ & $4.3-8.0$ & $15.6-16.0$ & $8-10$ \\
\hline 20 & 0.85 & 0.01 & 0.03 & 0.03 & 0.02 & 0.001 & 0.003 & 17.3 & 5.9 & 15.6 & 10 \\
\hline- & $0.85-1.85$ & $0.01-0.05$ & $0.03-0.13$ & $0.03-0.13$ & $0.02-0.08$ & $0.001-0.005$ & $0.002-0.012$ & $16.1-24.4$ & $4.5-8.0$ & $15.6-16.2$ & $8-10$ \\
\hline \multicolumn{12}{|c|}{ Fit to "Set 1 " line ratios ${ }^{b}$ with exponential beam correction } \\
\hline 10 & 0.39 & 0.09 & 0.23 & 0.24 & 0.14 & 0.009 & 0.022 & 17.3 & 7.9 & 16.2 & 10 \\
\hline - & $0.39-1.39$ & $0.00-0.24$ & $0.00-0.72$ & $0.00-1.01$ & $0.00-0.75$ & $0.000-0.022$ & $0.000-0.068$ & $15.5-44.5$ & $4.0-8.0$ & $14.0-16.8$ & $8-10$ \\
\hline 20 & 0.38 & 0.07 & 0.18 & 0.19 & 0.11 & 0.007 & 0.017 & 17.3 & 7.5 & 16.4 & 10 \\
\hline- & $0.38-1.37$ & $0.00-0.23$ & $0.00-0.63$ & $0.00-0.85$ & $0.00-0.58$ & $0.000-0.021$ & $0.000-0.060$ & $15.5-41.3$ & $4.0-8.0$ & $14.0-17.0$ & $8-14$ \\
\hline \multicolumn{12}{|c|}{ Fit to "Set 1 " line ratios ${ }^{b}$ with Gaussian beam correction ${ }^{c}$} \\
\hline 10 & 0.44 & 0.09 & 0.23 & 0.24 & 0.14 & 0.009 & 0.022 & 17.3 & 7.1 & 16.2 & 10 \\
\hline- & $0.44-1.44$ & $0.00-0.33$ & $0.00-0.85$ & $0.00-1.03$ & $0.00-0.75$ & $0.000-0.030$ & $0.000-0.080$ & $15.5-44.5$ & $4.0-8.0$ & $14.0-16.8$ & $8-10$ \\
\hline 20 & & 0.12 & 0.29 & 0.30 & 0.18 & 0.011 & 0.028 & 17 & 6.9 & 16.6 & 10 \\
\hline- & $0.43-1.43$ & $0.00-0.29$ & $0.00-0.74$ & $0.00-0.85$ & $0.00-0.59$ & $0.000-0.027$ & $0.000-0.070$ & $15.5-44.5$ & $4.0-8.0$ & $14.0-17.0$ & $8-14$ \\
\hline \multicolumn{12}{|c|}{ Fit to "other" sets of line ratios ${ }^{b}$ with exponential beam correction } \\
\hline 10 & 0.85 & 0.06 & 0.15 & 0.15 & 0.09 & 0.005 & 0.014 & 17. & 7.8 & 16.0 & 10 \\
\hline - & $0.85-1.84$ & $0.00-0.27$ & $0.00-0.91$ & $0.00-1.40$ & $0.00-1.20$ & $0.000-0.016$ & $0.000-0.056$ & $16.1-44.5$ & $4.0-8.0$ & $14.0-17.0$ & $8-20$ \\
\hline 20 & 0.83 & 0.05 & 0.12 & 0.12 & 0.07 & 0.004 & 0.011 & 17.3 & 8.0 & 16.2 & 10 \\
\hline- & $0.83-1.83$ & $0.00-0.24$ & $0.00-0.64$ & $0.00-0.78$ & $0.00-0.56$ & $0.000-0.014$ & $0.000-0.038$ & $16.1-27.3$ & $4.3-8.0$ & $14.0-17.0$ & $8-20$ \\
\hline $\begin{array}{c}\Delta V \\
\left(\mathrm{~km} \mathrm{~s}^{-1}\right)\end{array}$ & $\chi^{2}$ & ${ }^{12} \mathrm{CO}(1-0)$ & ${ }^{12} \mathrm{CO}(2-1)$ & $\begin{array}{c}\tau \\
{ }^{12} \mathrm{CO}(3-2) \\
\end{array}$ & ${ }^{12} \mathrm{CO}(4-3)$ & {$[\mathrm{CI}](1-0)$} & & $\begin{array}{l}T_{\text {kin }} \\
(\mathrm{K})\end{array}$ & $\begin{array}{c}\log \left(n_{\mathrm{H} 2}\right) \\
\left(\mathrm{cm}^{-3}\right)\end{array}$ & $\begin{array}{c}\log \left(N_{12 \mathrm{CO}}\right) \\
\left(\mathrm{cm}^{-2}\right)\end{array}$ & ${ }^{12} \mathrm{CO} / \mathrm{C}$ \\
\hline \multicolumn{12}{|c|}{ Fit to intensities with exponential beam correction } \\
\hline 10 & 0.55 & 0.02 & 0.06 & 0.06 & 0.04 & 0.05 & & 17.3 & 7.1 & 15.6 & 0.03 \\
\hline- & $0.55-1.54$ & $0.02-0.04$ & $0.06-0.10$ & $0.06-0.10$ & $0.03-0.06$ & $0.047-0.077$ & & $16.1-18.7$ & $5.1-8.0$ & $15.6-$ & $0.03-0.03$ \\
\hline 20 & 0.66 & 0.02 & 0.05 & 0.05 & 0.03 & 0.04 & & 17 & 6. & 15 & 0.03 \\
\hline- & $0.66-1.65$ & $0.01-0.05$ & $0.03-0.13$ & $0.03-0.13$ & $0.02-0.08$ & $0.023-0.097$ & & $16.1-18.7$ & $4.9-8.0$ & $15.6-16.2$ & $0.03-0.03$ \\
\hline \multicolumn{12}{|c|}{ Fit to intensities with Gaussian beam correction ${ }^{d}$} \\
\hline 10 & 0.75 & 0.04 & 0.10 & 0.10 & 0.06 & 0.08 & & 16.7 & 7.4 & 15.8 & 0.03 \\
\hline - & $0.75-1.75$ & $0.00-0.04$ & $0.07-0.10$ & $0.09-0.11$ & $0.06-0.07$ & $0.015-0.077$ & & $16.1-41.3$ & $4.0-8.0$ & $15.8-15.8$ & $0.03-0.10$ \\
\hline 20 & 0.66 & 0.02 & 0.05 & 0.05 & 0.03 & 0.04 & & 16.7 & 7.5 & 15.8 & 0.03 \\
\hline- & $0.66-1.66$ & $0.02-0.02$ & $0.04-0.05$ & $0.05-0.05$ & $0.03-0.03$ & $0.037-0.039$ & & $16.1-18.7$ & $4.9-8.0$ & $15.8-15.8$ & $0.03-0.03$ \\
\hline \multicolumn{12}{|c|}{ Fit to $[\mathrm{CI}] /{ }^{12} \mathrm{CO}$ ratios with exponential beam correction } \\
\hline 10 & 0.03 & 0.19 & 0.67 & 0.93 & 0.70 & 0.30 & & 26.3 & 4.5 & 16.8 & 0.06 \\
\hline- & $0.03-1.03$ & $0.00-0.94$ & $0.00-2.86$ & $0.00-4.32$ & $0.00-4.25$ & $0.000-1.927$ & & $16.1-44.5$ & $4.0-8.0$ & $14.0-17.6$ & $0.03-0.16$ \\
\hline 20 & 0.03 & 0.10 & 0.68 & 1.14 & 0.94 & 0.20 & & 38.3 & 4.1 & 17 & 0.10 \\
\hline- & $0.03-1.03$ & $0.00-0.97$ & $0.00-2.89$ & $0.00-3.88$ & $0.00-3.64$ & $0.000-1.546$ & & $16.1-44.5$ & $4.0-8.0$ & $14.0-17.8$ & $0.03-0.16$ \\
\hline \multicolumn{12}{|c|}{ Fit to $[\mathrm{CI}] /{ }^{12} \mathrm{CO}$ ratios with Gaussian beam correction ${ }^{e}$} \\
\hline 10 & & & & 1.43 & 1.08 & 0.48 & & & & 17.0 & 0.05 \\
\hline- & $0.01-1.01$ & $0.00-1.19$ & $0.00-3.88$ & $0.00-6.06$ & $0.00-6.40$ & $0.000-1.927$ & & $15.5-44.5$ & $4.0-8.0$ & $14.0-17.8$ & $0.03-0.25$ \\
\hline 20 & 0.01 & 0.24 & 1.10 & 1.70 & 1.46 & 0.34 & & 32.9 & 4.2 & 17.4 & 0.10 \\
\hline- & $0.01-1.01$ & $0.00-1.22$ & $0.00-3.93$ & $0.00-6.09$ & $0.00-6.58$ & $0.000-1.537$ & & $15.5-44.5$ & $4.0-8.0$ & $14.0-18.2$ & $0.03-0.25$ \\
\hline
\end{tabular}

Notes. The parameter ranges given in the second line for all sets of fits correspond to the full range found over the range in $\chi^{2}$ from $\chi_{\text {min }}^{2}$ to $\chi_{\min }^{2}+1$ i.e., marginalizing over the full range of posteriors. ${ }^{(a)}$ The intensity model gives a best-fit Gaussian FWHM $\theta_{\mathrm{s}}=31^{\prime \prime}\left(\Delta V=10 \mathrm{~km} \mathrm{~s}{ }^{-1}\right)$, and $\theta_{\mathrm{s}}=29$ " $\left(\Delta V=20 \mathrm{~km} \mathrm{~s}^{-1}\right) .{ }^{(b)}$ "Set 1 " was taken with the ratios relative to the adjacent lower- $J$ line: $\left[{ }^{12} \mathrm{CO}(2-1) /{ }^{12} \mathrm{CO}(1-0),{ }^{12} \mathrm{CO}(3-2) /{ }^{12} \mathrm{CO}(2-1)\right.$, $\left.{ }^{12} \mathrm{CO}(4-3) /{ }^{12} \mathrm{CO}(3-2),{ }^{13} \mathrm{CO}(1-0) /{ }^{12} \mathrm{CO}(1-0),{ }^{13} \mathrm{CO}(2-1) /{ }^{13} \mathrm{CO}(1-0)\right]$. The "other" sets of line ratios with various $J$ transitions in the numerators/denominators give virtually identical results so we report here only a representative example with $\left[{ }^{12} \mathrm{CO}(2-1) /{ }^{12} \mathrm{CO}(1-0),{ }^{12} \mathrm{CO}(3-2) /{ }^{12} \mathrm{CO}(1-\right.$ $\left.0),{ }^{12} \mathrm{CO}(4-3) /{ }^{12} \mathrm{CO}(1-0),{ }^{13} \mathrm{CO}(2-1) /{ }^{13} \mathrm{CO}(1-0),{ }^{12} \mathrm{CO}(2-1) /{ }^{13} \mathrm{CO}(2-1)\right] .{ }^{(c)}$ The Set 1 line ratio models give a best-fit Gaussian FWHM $\theta_{\mathrm{s}}=27{ }^{\prime \prime}$ $\left(\Delta V=10 \mathrm{~km} \mathrm{~s}^{-1}\right)$, and $\theta_{\mathrm{s}}=26^{\prime \prime}\left(\Delta V=20 \mathrm{~km} \mathrm{~s}^{-1}\right)$. ${ }^{(d)}$ The $[\mathrm{CI}](1-0)$ line ratio models give a best-fit Gaussian FWHM $\theta_{\mathrm{s}}=26^{\prime \prime}\left(\Delta V=10 \mathrm{~km} \mathrm{~s}^{-1}\right)$, and $\theta_{\mathrm{s}}=27^{\prime \prime}\left(\Delta V=20 \mathrm{~km} \mathrm{~s}^{-1}\right)$. ${ }^{(e)}$ The $[\mathrm{CI}](1-0)$ line ratio models give a best-fit Gaussian FWHM $\theta_{\mathrm{s}}=23^{\prime \prime}\left(\Delta V=10 \mathrm{~km} \mathrm{~s}^{-1}\right)$, and $\theta_{\mathrm{s}}=24^{\prime \prime}$ $\left(\Delta V=20 \mathrm{~km} \mathrm{~s}^{-1}\right)$.

allowed by the models (for $\left.\chi^{2}=\chi^{2}(\min )+1\right)$. The similar ${ }^{12} \mathrm{CO}$ and ${ }^{13} \mathrm{CO}$ slopes for NGC 1140 (the data points follow parallel lines) suggest that even the ${ }^{12} \mathrm{CO}$ emission may be optically thin, as also implied by the best-fit results in Table 6. The CO gas is in an excited state, more similar to the center of M 82 (Weiß et al. 2005) than to the Milky Way (e.g., Carilli \& Walter 2013). This 


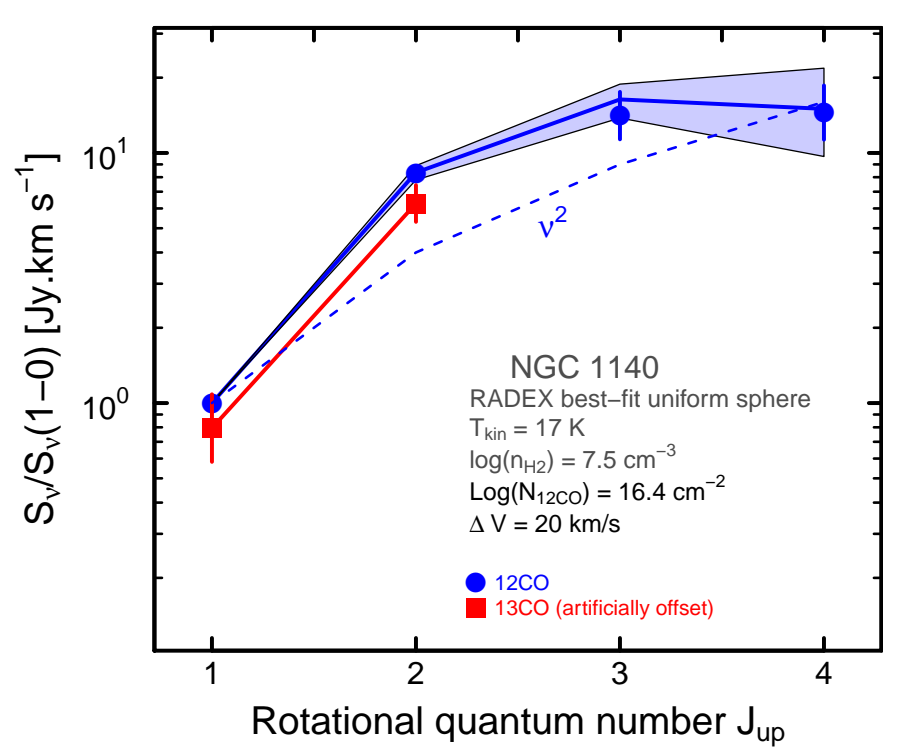

Fig. 13. RADEX best-fit model compared to observations for the CO excitation ladder of NGC 1140; the observations have been corrected for beam size as described in Sect. 4, and normalized to the $\mathrm{CO}(1-0)$ flux. The observations are shown as filled blue circles $\left({ }^{12} \mathrm{CO}\right)$ and red squares $\left({ }^{13} \mathrm{CO}\right)$, and the range of the allowed RADEX models by the light-blue region (for ${ }^{12} \mathrm{CO}$ ); ${ }^{13} \mathrm{CO}$ points have arbitrarily offset relative to ${ }^{12} \mathrm{CO}$ for clarity. The dashed curve shows the $v^{2}$ behavior (here normalized to $J=1$ ) expected for optically-thick emission.

is not a surprising result given the population of SSCs in the nuclear region of NGC 1140.

The low optical depths of even the ${ }^{12} \mathrm{CO}$ lines steepen the excitation ladder at low $J$, steeper than $v^{2}$ as would be expected for thermalized, optically thick lines. The shape is an indirect consequence of the normalization to $J=1-0$, due to the increase of $\tau_{\mathrm{CO}}$ with increasing $J$ up to the inflection in the excitation. Because ${ }^{12} \mathrm{CO}(1-0)$ has the smallest $\tau$ of the ${ }^{12} \mathrm{CO}$ transitions, with a different normalization, it would drop below the $v^{2}$ trend. The results of the RADEX fit (see Table 6) are telling us that the $J=4$ level is sparsely populated because of the decreased $\tau_{\mathrm{CO}}$ relative to the lower- $J$ transitions; this produces the flattening in the excitation ladder.

\subsubsection{Atomic carbon}

We have performed additional RADEX fits to the ${ }^{12} \mathrm{CO}$ and [CI](1-0) emission, both with intensities as in Eq. (4) and with ratios (Eq. (5)). Fitted parameters include, as before, $\left(n_{\mathrm{H} 2}, T_{\text {kin }}, N_{\mathrm{CO}}\left({ }^{12} \mathrm{CO}\right), F\right)$, but now $\left[{ }^{12} \mathrm{CO}\right] /\left[{ }^{12} \mathrm{C}\right]$, rather than $\left[{ }^{12} \mathrm{CO}\right] /\left[{ }^{13} \mathrm{CO}\right]$. Here, again, the implicit assumption is that atomic carbon and $\mathrm{CO}$ occupy the same volume, and are uniformly intermixed within an ISM of a single $\mathrm{H}_{2}$ kinetic temperature and volume density; such an assumption is consistent with the findings of Requena-Torres et al. (2016) for HII regions in the SMC and of Okada et al. (2015) for the LMC. The results of the fits are given in Table 6 and shown graphically in Fig. 14 where the $\chi^{2}$ surfaces (in logarithmic terms) are shown as a function of $n_{\mathrm{H} 2}$ and $N_{\mathrm{CI}(1-0)}$ column density. As for the previous $\mathrm{CO}$ analysis, we prefer the $\Delta V=20 \mathrm{~km} \mathrm{~s}^{-1}$ fits, roughly the mean component line width observed in NGC 1140.

The intensity fit of the ${ }^{12} \mathrm{CO}$ and $[\mathrm{CI}]$ emission gives parameters very similar to the $\mathrm{CO}$ fit, although the inferred $n_{\mathrm{H} 2}$ for the $[\mathrm{CI}](1-0)$ fit is lower. On the other hand, the fit to the $[\mathrm{CI}](1-0)$ ratios (with ${ }^{12} \mathrm{CO}$ ) gives higher $T_{\text {kin }}$, lower $n_{\mathrm{H} 2}$, and 25 times higher $N_{\mathrm{CO}}$, higher than any other fit we performed. Folding in the best-fit $\left[{ }^{12} \mathrm{CO}\right] /\left[{ }^{12} \mathrm{C}\right]$ abundance ratio, which varies by a factor of $\sim 3$ between the two fits, shows that the best-fit column density of atomic carbon $N_{\mathrm{C}}$ is also different for both fits: $\sim 10^{17.3} \mathrm{~cm}^{-2}$ in the intensity case and $\sim 10^{18.2} \mathrm{~cm}^{-2}$ for the ratios. Consequently, the filling factors for the two fits also differ. The best-fit $F$ for the [CI] intensity fits is 0.10 as for (the intensity fitted) $\mathrm{CO}$, but for the $[\mathrm{CI}]$ ratio fits, it is much lower, $F \lessgtr 0.01$. This is because the higher $N_{\mathrm{CO}}$ requires a lower filling factor to accommodate the RADEX predictions with the observations. We found a similar behavior in Sect. 7.3 for the CO ratio fits relative to the intensity ones. The reasons for the disagreement are unclear, although the low values of $\chi^{2}$ for all the [CI] fits make it unlikely that the model is failing to capture the physical conditions in the clouds. In any case, these values of $F$ are between 2 and 20 times lower than those found for [CI] emission in the Magellanic Clouds by Pineda et al. (2017); however their observations probe physical scales more than 50 times smaller, so a comparison is not straightforward.

Independently of the disagreement, our RADEX fits for [CI] all find that the atomic carbon column density must be larger than $N_{\mathrm{CO}}$, implying a $\left[{ }^{12} \mathrm{CO}\right] /\left[{ }^{12} \mathrm{C}\right]$ abundance of $<1$. For the intensity fit, with lower $N_{\mathrm{CO}}$, this requires a very low $\left[{ }^{12} \mathrm{CO}\right] /\left[{ }^{12} \mathrm{C}\right]$ of $\sim 0.03$, while for the ratio fit with higher $N_{\mathrm{CO}}$, $\left[{ }^{12} \mathrm{CO}\right] /\left[{ }^{12} \mathrm{C}\right] \sim 0.1$. This would indicate that atomic carbon is at least 10 times more abundant than ${ }^{12} \mathrm{CO}$ in NGC 1140. Such a result would be consistent with the theoretical predictions of Bialy \& Sternberg (2015) for interstellar ion-molecule gas-phase chemistry in galaxies; their models predict that $\left[{ }^{12} \mathrm{CO}\right] /\left[{ }^{12} \mathrm{C}\right]$ abundance ratios decrease with decreasing metallicity and with higher ionization parameters. On the other hand, observational studies of luminous (metal-rich) galaxies found that atomic carbon is less abundant than $\mathrm{CO},\left[{ }^{12} \mathrm{CO}\right] /\left[{ }^{12} \mathrm{C}\right] \sim 4-$ 8 (e.g., Israel 2009; Israel et al. 2015). However, the observed $[\mathrm{CI}](1-0) /{ }^{12} \mathrm{CO}(4-3)$ ratio (in flux units) for NGC $1140(\sim 0.7)$ is more than $4 \sigma$ higher than the mean of the galaxies observed by Israel et al. (2015), so the relatively high abundance of atomic carbon is perhaps not altogether surprising. Although our $[\mathrm{CI}](1-0)$ detection is marginal $(\sim 3 \sigma$, see Table 4$)$, to reduce the inferred $\left[{ }^{12} \mathrm{CO}\right] /\left[{ }^{12} \mathrm{C}\right]$ ratio to unity or less would require a reduction in the $[\mathrm{CI}](1-0)$ intensity by more than a factor of 10 ; this seems unlikely given the trends shown in Fig. 5.

Independent one-zone model fitting of the ${ }^{12} \mathrm{CO},{ }^{13} \mathrm{CO}$, and $[\mathrm{CI}](1-0)$ emission of NGC 1140 gives similar physical conditions. Specifically, by fitting a vast grid of $\mathrm{LVG}$ models (Weiß et al. 2007) to the NGC 1140 data, and considering dust continuum, and molecular and atomic abundances relative to $\mathrm{H}_{2}$, we obtain high densities $\left(n_{\mathrm{H} 2} \gtrsim 10^{5} \mathrm{~cm}^{-3}\right)$, cool temperatures $\left(T_{\text {kin }} \sim 20 \mathrm{~K}\right)$, low $\left[{ }^{12} \mathrm{CO}\right] /\left[{ }^{13} \mathrm{CO}\right]$ abundance ratios $(\sim 13)$, low $\left[{ }^{12} \mathrm{CO}\right] /\left[{ }^{12} \mathrm{C}\right]$ abundance ratios $(\sim 0.13)$, and moderate column densities $\left(N_{\mathrm{CO}} \sim 5 \times 10^{16} \mathrm{~cm}^{-2}\right)$, similar to the RADEX results. However, with these models, a "warm" solution with $T_{\text {kin }} \sim 52 \mathrm{~K}$ is also possible. This solution would result in higher abundance ratios $\left(\left[{ }^{12} \mathrm{CO}\right] /\left[{ }^{13} \mathrm{CO}\right] \sim 36,\left[{ }^{12} \mathrm{CO}\right] /\left[{ }^{12} \mathrm{C}\right] \sim 0.3\right)$, and higher $N_{\mathrm{CO}}$ $\left(\sim 7 \times 10^{17} \mathrm{~cm}^{-2}\right)$. Nevertheless, in this case there would be an unrealistically low gas-to-dust mass ratio (GDR), $\sim 62$; this is lower than even the Milky Way (GDR 100, Draine et al. 2007), which is unlikely considering the low metallicity of NGC 1140. With $Z / Z_{\odot} \sim 0.3$ for NGC 1140, and given the linear trend of GDR with metallicity in this abundance range (e.g., Rémy-Ruyer et al. 2014), we would expect a GDR of 300, not far from the GDR of $\sim 380$, inferred from the "cool" solution obtained with the Weiß et al. (2007) LVG models. Thus, 

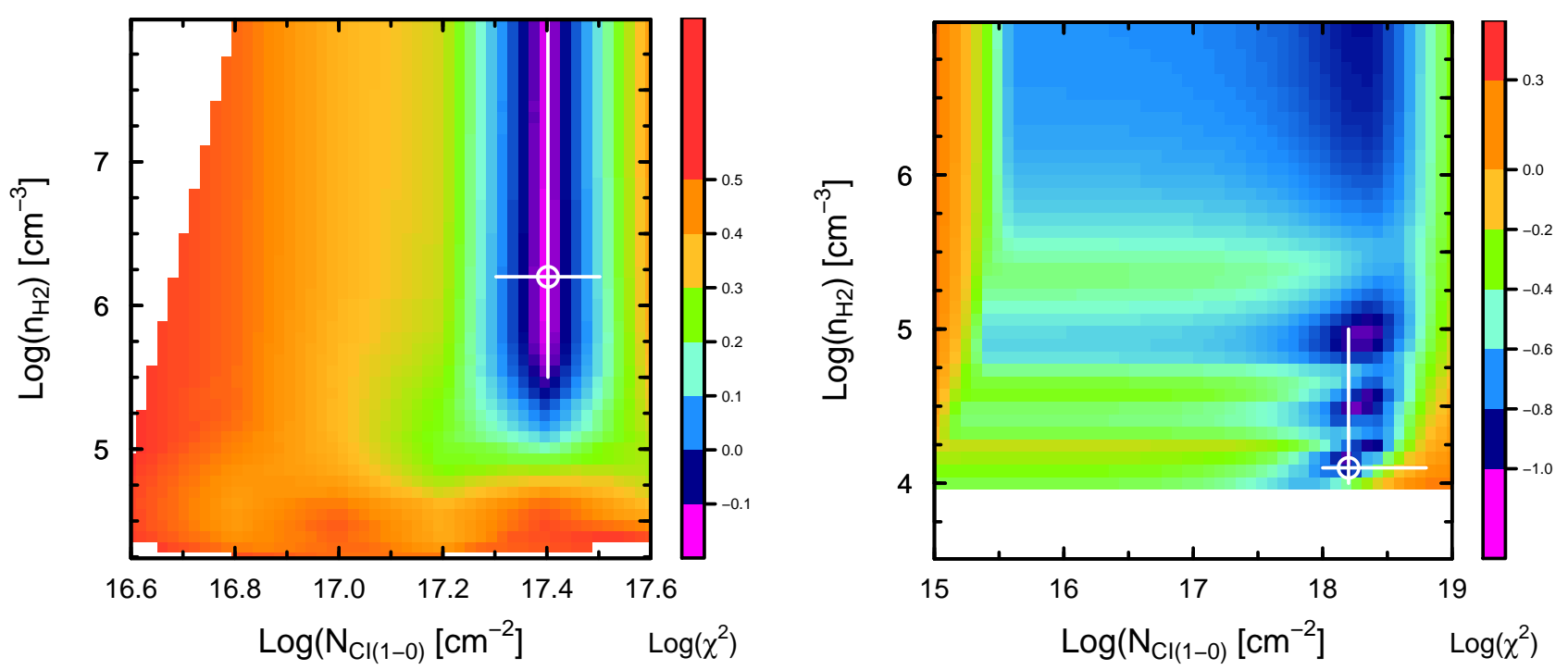

Fig. 14. RADEX fit of the $\chi^{2}$ (logarithmic) surface of the $[\mathrm{CI}] /{ }^{12} \mathrm{CO}$ fits as a function of $n_{\mathrm{H} 2}$ and $N_{\mathrm{CI}(1-0)}$; the left panel shows the intensity fits (Eq. (4)) and the right the ratio ones (Eq. (5)). The RADEX models shown here have $\Delta V=20 \mathrm{~km} \mathrm{~s}^{-1}$. The associated $\log \left(\chi^{2}\right)$ values are shown as a side-bar color table. The best-fit values are shown as an open (white) circle; the error bars shown consider a slightly more limited range in $\chi^{2}$ respect to Table 6.

the "cool" solution with $T_{\text {kin }} \sim 20 \mathrm{~K}$ seems more likely for NGC 1140.

The "warm" solution with the Weiß et al. (2007) LVG models for ${ }^{12} \mathrm{CO},{ }^{13} \mathrm{CO}$, and $[\mathrm{CI}](1-0)$ is not far from the "warm" solution (with $T_{\text {kin }} \sim 38 \mathrm{~K}$ ) that emerged from the RADEX fitting of $[\mathrm{CI}](1-0)$ and ${ }^{12} \mathrm{CO}$ line ratios (see Table 6). However, our RADEX models for ${ }^{13} \mathrm{CO}$ and ${ }^{12} \mathrm{CO}$ do not allow the higher $T_{\text {kin }}$ found with the Weiß et al. (2007) models; we obtain $\chi^{2}>10$ for $T_{\text {kin }}>46 \mathrm{~K}$, and a mean $\chi^{2}$ of $\sim 6-7$ for $40 \leq T_{\text {kin }} \leq 46 \mathrm{~K}$, compared to $\chi^{2}$ values of $<1$ for our best fits. Based on the much lower $\chi^{2}$ values for the $\mathrm{CO}$ fits with cool $T_{\mathrm{kin}}$, we do not consider further the "warm" RADEX fits. Observations of higher- $J$ CO lines and $[\mathrm{CI}](2-1)$ would help settle this potential ambiguity.

Combined with the $\left[{ }^{12} \mathrm{CO}\right] /\left[{ }^{13} \mathrm{CO}\right]$ abundance ratio of $\sim 10$ 12 found above (see also Sect. 7.1), this would imply a $\left[{ }^{12} \mathrm{C}\right] /\left[{ }^{13} \mathrm{CO}\right]$ abundance ratio of $\gtrsim 100-300$, within the range of values found by Israel et al. (2015) for galaxies with low $\mathrm{CO} /[\mathrm{CI}](1-0)]$ ratios. However, this may be a specious comparison because of the unusually low $\left[{ }^{12} \mathrm{CO}\right] /\left[{ }^{13} \mathrm{CO}\right]$ abundance ratio which, in some sense, compensates for the extremely low $\left[{ }^{12} \mathrm{CO}\right] /\left[{ }^{12} \mathrm{C}\right]$ found here for NGC 1140 . As already mentioned, we discuss further the low $\left[{ }^{12} \mathrm{CO}\right] /\left[{ }^{13} \mathrm{CO}\right]$ abundance ratio found for NGC 1140 in Sect. 8.1.

\subsubsection{Modeling HCN with RADEX}

We did not detect $\mathrm{HCN}(1-0)$ in NGC 1140, even though the RADEX best fit suggests that there is dense gas in this galaxy. Figure 15 shows the predictions of RADEX models for the $\mathrm{HCN}(1-0) /{ }^{12} \mathrm{CO}(1-0)$ line ratio plotted against $\mathrm{H}_{2}$ volume density, $n_{\mathrm{H} 2}$ and assuming $[\mathrm{CO}] /[\mathrm{HCN}]=10^{5}$. The implicit assumption here, almost certainly incorrect, is that the filling factor of the emitting gas is the same in both transitions. Figure 15 suggests that the abundance ratio $[\mathrm{CO}] /[\mathrm{HCN}]$ must be high $\gtrsim 10^{5}$. Relative to the best-fit RADEX model for NGC 1140 , $[\mathrm{CO}] /[\mathrm{HCN}]=10^{5}$ corresponds to an $\mathrm{HCN}$ column density of $\sim 2 \times 10^{11} \mathrm{~cm}^{-2}$, an order of magnitude lower than in diffuse clouds in the Galaxy (Liszt \& Lucas 2001).

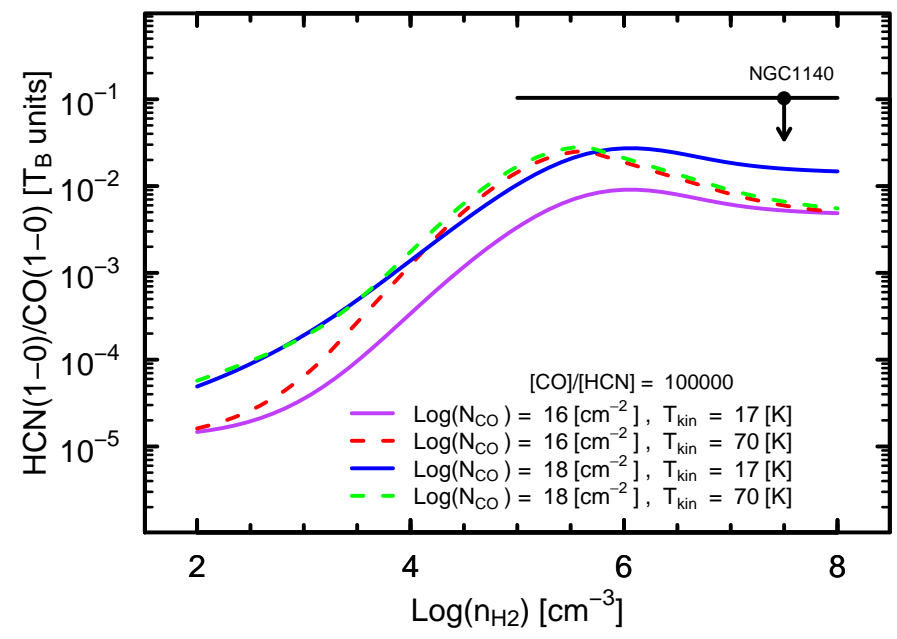

Fig. 15. RADEX models of $\mathrm{HCN}(1-0) /{ }^{12} \mathrm{CO}(1-0)$ line ratios plotted against $n_{\mathrm{H} 2}$, and assuming [CO]/[HCN] $=10^{5}$. Here the $\Delta V=20 \mathrm{~km} \mathrm{~s}^{-1}$ models are illustrated. The upper limit $\mathrm{HCN}(1-0) /{ }^{12} \mathrm{CO}(1-0)$ ratio for NGC 1140, shown as a (black) filled circle, has not been corrected for beam dilution because of the similar beam sizes for the two transitions. The approximate best-fit RADEX model for NGC 1140 is shown as a (purple) solid curve that is consistent with the non-detection of $\mathrm{HCN}(1-0)$. Also shown are three additional parameter combinations of ${ }^{12} \mathrm{CO}$ column density $N_{12 \mathrm{CO}}$ and volume density $n_{\mathrm{H} 2}$.

The upper limit (UL) for the $\mathrm{HCN}(1-0) /{ }^{12} \mathrm{CO}(1-0)$ line temperature ratio of $\mathrm{NGC} 1140$ is $\sim 0.1$, higher than the detected $\mathrm{HCN}(1-0) /{ }^{12} \mathrm{CO}(1-0)$ ratio of $\sim 0.05$ in the prominent star-formation region N113 of the LMC (Wang et al. 2009). Thus, even after $18 \mathrm{~h}$ with the IRAM $30 \mathrm{~m}$, our observations are not able to constrain the HCN content of NGC 1140. More observations of dense-gas tracers are needed at low metallicity, especially outside the Local Group where conditions can be even more extreme than in the massive star-forming regions in the Magellanic Clouds. 


\section{Discussion}

Unlike some previous work covering a similar range of $\mathrm{CO} J$ values (e.g., Meier et al. 2001; Israel 2005; Nikolić et al. 2007), our one-zone non-LTE models give very good fits to the CO and atomic carbon emission in NGC 1140. Part of this success is almost certainly due to the lack of high- $J$ transitions in our data set; we are fitting only the cool, dense clouds in this galaxy, while there may be a substantial contribution of warmer, more diffuse gas. It may also be a consequence of the extreme conditions in this galaxy. None of the six dwarf galaxies observed by Cormier et al. (2014) in ${ }^{12} \mathrm{CO}(1-0)$ and ${ }^{12} \mathrm{CO}(2-1)$ have $R_{21}>1$, independently of the beam size of the observations or the upper limits. The $\mathrm{N} 113$ region in the LMC has $R_{21} \lessgtr 1$ after beam deconvolution $\left(R_{21} \sim 1.2\right.$ before, see Wang et al. 2009), and Minamidani et al. (2008) found maximum values of $R_{31} \gtrsim 1$ in only a handful of the $33 \mathrm{LMC}$ regions observed. These ratios are much higher in NGC $1140\left(R_{21}=2.1, R_{31}=1.7\right.$, see Figs. 3 , 4 , and Table A.1), independently of the beam corrections applied. Because of these high line ratios, and the implied more extreme physical conditions dominating the ISM of this galaxy, one-zone models may be more effective.

The CO excitation in NGC 1140 is starburst-like, similar to M 82, but as mentioned before, this is not particularly surprising given the presence of at least 6 SSCs within its central region (de Grijs et al. 2004; Moll et al. 2007). However, unlike M 82, where the gas is warm $\left(T_{\text {kin }} \sim 60-200 \mathrm{~K}\right)$ and not particularly dense $\left(n_{\mathrm{H} 2} \sim 10^{3} \mathrm{~cm}^{-3}\right.$, Weiß et al. 2005; Mühle et al. 2007), in NGC 1140 the gas is dense and cool. Given the relatively high excitation, it is not straightforward to understand why the $\mathrm{CO}$ has not been photo-dissociated through the intense radiation field, although as discussed below, the dense, small-filling factor clouds may be one part of the explanation. High atomic gas columns may also contribute to self-shielding as discussed below and suggested by Wong et al. (2009) for the LMC.

Finding evidence for cool, dense gas, $n_{\mathrm{H} 2} \gtrsim 10^{6} \mathrm{~cm}^{-3}$ in NGC 1140 was unexpected. However, at least one other lowmetallicity galaxy outside the Local Group shows similar properties. In Haro 11, PDR modeling (Cormier et al. 2012, 2014) also suggests that the molecular gas is dense, with hydrogen densities $n_{H} \sim 10^{5-6} \mathrm{~cm}^{-3}$. The Cormier et al. (2014) PDR model for Haro 11 implies that $\mathrm{CO}$ emission is produced at intermediate depths within the cloud ( $3<A_{V}<6 \mathrm{mag}$ ), and for larger $A_{V}$, the gas temperature is $\sim 30 \mathrm{~K}$, somewhat warmer than the (non-LTE) gas temperature $T_{\text {kin }}$ of $\sim 17 \mathrm{~K}$ we find for NGC 1140 .

Another unexpected result is the low optical depth of the ${ }^{12} \mathrm{CO}$ transitions. Our RADEX fits (see Table $6, \Delta V=20 \mathrm{~km} \mathrm{~s}^{-1}$ ) suggest that the ${ }^{12} \mathrm{CO}$ and ${ }^{13} \mathrm{CO}$ transitions have a maximum $\tau_{\mathrm{CO}} \sim 0.2$ for ${ }^{12} \mathrm{CO}(3-2)$. The best fit of ${ }^{12} \mathrm{CO}$ with [CI] gives higher optical depths, with $\tau_{\mathrm{CO}} \sim 1$ for ${ }^{12} \mathrm{CO}(3-2)$ but lower for the other transitions. Apparently even these low optical depths allow sufficient self-shielding for detectable ${ }^{12} \mathrm{CO}$ and ${ }^{13} \mathrm{CO}$ emission. This could be possible because the clouds are dense and occupy only a small fraction of the macroscopic source size (see Sect. 7.3.1).

It could also be that high column densities are contributing to the shielding of the molecular gas. Hunter et al. (1994a) find a peak HI column $N_{\mathrm{HI}}$ of $2.4 \times 10^{21} \mathrm{~cm}^{-2}$ (averaged over a $30^{\prime \prime} \times 37^{\prime \prime}$ beam), 10 times higher than the $N_{\mathrm{H} 2}$ of $2 \times$ $10^{20} \mathrm{~cm}^{-2}$ estimated from $N_{\mathrm{CO}} \sim 10^{16} \mathrm{~cm}^{-2}$, assuming a roughly solar abundance ratio $\left[\mathrm{H}_{2}\right] /[\mathrm{CO}] \sim 5 \times 10^{-5}$ (e.g., Sakamoto 1999). Even the disk-averaged $N_{\mathrm{HI}}$ in NGC 1140 of $\sim 10^{21} \mathrm{~cm}^{-2}$ (Fumagalli et al. 2010) is 5 times higher than the $\mathrm{H}_{2}$ column that we would infer from $N_{\mathrm{CO}}$. As proposed by Wong et al. (2009), high HI columns may even be necessary (but not sufficient) for the detection of $\mathrm{CO}$ in low-metallicity galaxies. However, the LVG abundance fits using the models of Weiß et al. (2007) indicate that the $[\mathrm{CO}] /\left[\mathrm{H}_{2}\right]$ abundance ratio in NGC 1140 is extremely low: $\sim 100$ times lower than solar for the "cool" solution ([CO]/[H $\left.\mathrm{H}_{2}\right] \sim 8 \times 10^{-7}$ ). This would imply that the $\mathrm{H}_{2}$ columns could be comparable to, if not exceed, the HI, thus dominating the column density contribution necessary for self-shielding.

Such a low $[\mathrm{CO}] /\left[\mathrm{H}_{2}\right]$ abundance and cold $T_{\text {kin }}$ in NGC 1140 could be the result of a high level of photo-dissociation of $\mathrm{CO}$, leaving only the densest, best shielded cores. This is a similar situation to ammonia $\left(\mathrm{NH}_{3}\right)$ in the LMC where it is only detected in $\mathrm{N} 159 \mathrm{~W}$ (Ott et al. 2010). In this region, the $\mathrm{NH}_{3}$ is cold with $T_{\text {kin }} \sim 16 \mathrm{~K}$, and has very low abundance; $\mathrm{NH}_{3}$ abundance is between 1.5 and 5 orders of magnitude lower than observed in Galactic star-forming regions, but similar to that found in the late-stage starburst M 82 (Weiß et al. 2001). $\mathrm{NH}_{3}$ is particularly sensitive to UV radiation (Sato \& Lee 1983), but formaldehyde $\left(\mathrm{H}_{2} \mathrm{CO}\right)$ is less vulnerable to photo-dissociation than $\mathrm{NH}_{3}$, and in $\mathrm{N} 159 \mathrm{~W}$ is warmer, $T_{\text {kin }} \sim 30-35 \mathrm{~K}$ (Tang et al. 2017). The implication is that $\mathrm{H}_{2} \mathrm{CO}$ is sampling regions that are more exposed to radiation of young massive stars, while $\mathrm{NH}_{3}$ resides in the most shielded knots of dense gas (Tang et al. 2017). The stronger radiation field in NGC 1140 from the concentration of SSCs would annihilate $\mathrm{NH}_{3}$, and possibly also $\mathrm{H}_{2} \mathrm{CO}$, but $\mathrm{CO}$ could survive, thus playing the role that $\mathrm{NH}_{3}$ plays in the $\mathrm{LMC}$, tracing the surviving cool pockets of dense gas.

Such a scenario may occur in any specific low-metallicity star-forming region. That it is seen over a $\sim 2 \mathrm{kpc}$ area of a galaxy with six SSCs requires that the evaporation of dense molecular gas occurs roughly simultaneously. Thus, galaxies like NGC 1140 are probably rare. Consistently with observations, the starburst must also be either quite young (all activity is occurring at almost the same time) or the last one of several possibly very short starburst episodes (e.g., de Grijs et al. 2004; Moll et al. 2007). With 6 SSCs, all of them clearly surpassing 30 Doradus in the LMC, this last starburst episode must then be at least (if not the only one) the strongest one. More extensive observations of molecular tracers are necessary to test this scenario, and constrain models of $\mathrm{CO} / \mathrm{H}_{2}$ abundance ratios in NGC 1140 , and, more generally, in metal-poor starbursts.

Our RADEX fits with ${ }^{12} \mathrm{CO}$ and $[\mathrm{CI}]$ also give an unusually high atomic carbon abundance (see Sect. 7.3.3), $\mathrm{C} / \mathrm{CO} \gtrsim$ 10. This result is premised on the assumption that $[\mathrm{CI}]$ and $\mathrm{CO}$ occupy the same volume in a cloud characterized by a single kinetic temperature and volume density (e.g., Okada et al. 2015; Requena-Torres et al. 2016). Such a high [CI] abundance relative to $\mathrm{CO}$ is more extreme than found in the SMC with the LVG analysis of Requena-Torres et al. (2016); there the clouds tend to be less dense $\left(n_{\mathrm{H} 2} \sim 10^{4} \mathrm{~cm}^{-3}\right)$, and warmer $\left(T_{\text {kin }} \sim 30-50 \mathrm{~K}\right)$, although of similar CO column density $\left(N_{\mathrm{CO}} \sim 2-4 \times 10^{16} \mathrm{~cm}^{-2}\right.$, see also Nikolić et al. 2007). Spatially-resolved observations of [CI] and CO in NGC 1140 would help understand whether the high $[\mathrm{CI}]$ abundance we find is dictated by a different spatial distribution relative to $\mathrm{CO}$, unlike what is observed in the Magellanic Clouds.

\section{1. ${ }^{13} \mathrm{CO}$ charge-exchange reactions and fractionation}

Another surprising result from our RADEX analysis of NGC 1140 is the unusually low $\left[{ }^{12} \mathrm{CO}\right] /\left[{ }^{13} \mathrm{CO}\right]$ abundance ratio, $\sim 8-20$, lower even than the value of $\sim 24$ found in the Galactic Center (Langer \& Penzias 1990). Isotopic ratios are generally governed by two competing processes: photo-selective 


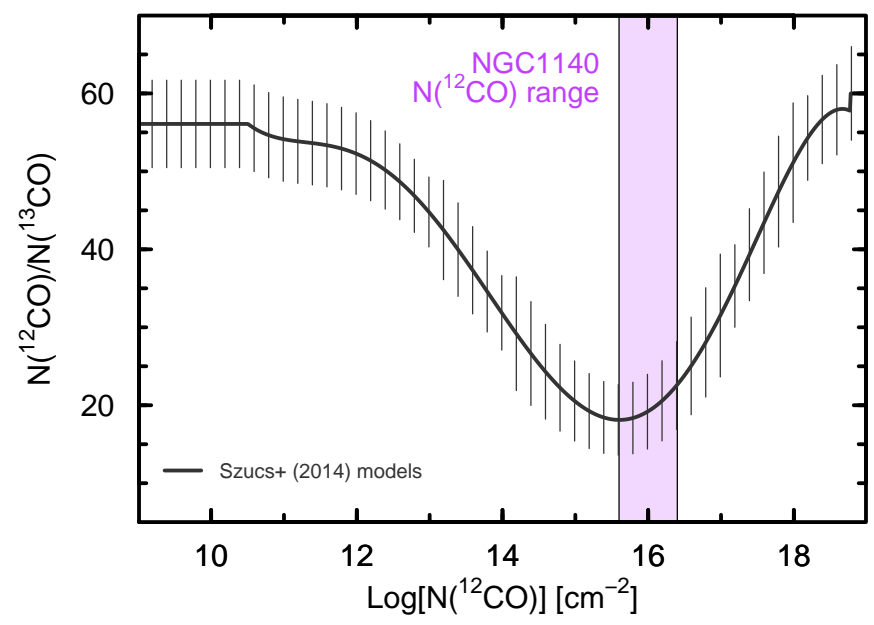

Fig. 16. ${ }^{12} \mathrm{CO} /{ }^{13} \mathrm{CO}$ abundance ratio plotted against $N\left({ }^{12} \mathrm{CO}\right)$. The shaded rectangle shows the ranges of values from RADEX fits $\left(\Delta V=20 \mathrm{~km} \mathrm{~s}^{-1}\right)$ of NGC 1140 . The solid line corresponds to the models of the ${ }^{12} \mathrm{CO} /{ }^{13} \mathrm{CO}$ abundance ratio vs. the ${ }^{12} \mathrm{CO}$ column density by Szúcs et al. (2014). The error bars for these models (vertical black lines) are (very roughly) estimated from their Fig. 8.

dissociation that would tend to decrease the relative ${ }^{13} \mathrm{CO}$ abundance, and low-temperature carbon isotope exchange reactions that would tend to increase it.

Low $\left[{ }^{12} \mathrm{CO}\right] /\left[{ }^{13} \mathrm{CO}\right]$ abundance ratios are typically found in older intermediate-mass stellar populations ( $\gtrsim 500 \mathrm{Myr})$, in which ${ }^{13} \mathrm{C}$ is produced in nuclear reactions in the cores of asymptotic giant branch (AGB) stars, and "dredged up" through convective mixing where it is ejected into the ISM in stellar winds (e.g., Milam et al. 2005, 2009). However, the SSCs in NGC 1140 are quite young, with ages ranging from $\sim 5$ to $12 \mathrm{Myr}$, and the overall starburst is younger than $~ 55$ Myr (de Grijs et al. 2004; Moll et al. 2007). These young ages make it unlikely that the isotopic ratio in NGC 1140 has been lowered by stellar reprocessing (e.g., Boothroyd \& Sackmann 1999; Pavlenko et al. 2003; Henkel et al. 2014).

Instead, the extremely low ${ }^{12} \mathrm{CO} /{ }^{13} \mathrm{CO}$ abundance ratio for NGC 1140 could be explained by charge-exchange reactions, i.e., fractionation (e.g., Watson et al. 1976). Isotopic selective photo-dissociation of CO is effective only in diffuse gas $\left(n_{\mathrm{H} 2} \lesssim 100 \mathrm{~cm}^{-3}\right)$; in denser regions with higher column density but moderate extinction ( $1 \mathrm{mag} \lesssim A_{V} \lesssim 3 \mathrm{mag}$ ), fractionation reactions become important:

${ }^{13} \mathrm{C}^{+}+{ }^{12} \mathrm{CO} \leftrightharpoons{ }^{12} \mathrm{C}^{+}+{ }^{13} \mathrm{CO}+\Delta E(=35 \mathrm{~K})$.

At intermediate $N_{\mathrm{CO}}$ (and $T \lesssim 35 \mathrm{~K}$ ), the rightmost (exothermic) reaction dominates, enhancing ${ }^{13} \mathrm{CO}$ and leading to a reduced $\left[{ }^{12} \mathrm{CO}\right] /\left[{ }^{13} \mathrm{CO}\right]$ abundance ratio. Thus, if molecular gas in low-metallicity galaxies is dense and cool, but with moderate $N_{\text {CO }}$ as in NGC 1140, it is possible that fractionation drives low ${ }^{12} \mathrm{CO} /{ }^{13} \mathrm{CO}$ ratios.

The low value of $\left[{ }^{12} \mathrm{CO}\right] /\left[{ }^{13} \mathrm{CO}\right]$ we obtain for NGC 1140 is roughly consistent with (although slightly higher than) the trends of $\mathrm{CO}$ column density and ${ }^{13} \mathrm{CO}$ fractionation found by Röllig \& Ossenkopf (2013) and Szúcs et al. (2014). Figure 16 shows the trend of ${ }^{12} \mathrm{CO} /{ }^{13} \mathrm{CO}$ abundance ratio and ${ }^{12} \mathrm{CO}$ column density given by Szúcs et al. (2014); the range of $N\left({ }^{12} \mathrm{CO}\right)$ for NGC 1140 as estimated by the best-fit RADEX (and LTE) models is shown as a shaded rectangle. Szúcs et al. (2014) show that there is a "sweet spot" in $N_{\mathrm{CO}}$ for maximizing the fractionation process, as long as the temperature barrier of $\sim 35 \mathrm{~K}$ is not exceeded. Interestingly, the best-fit $T_{\text {kin }}$ for NGC 1140 is $\sim 16-$ $18 \mathrm{~K}$ (see Fig. 11 and Table 6), consistent with the low temperatures necessary for optimizing ${ }^{13} \mathrm{CO}$ fractionation. In NGC 1140 , the low $\mathrm{X}\left({ }^{12} \mathrm{CO}\right) / \mathrm{X}\left({ }^{13} \mathrm{CO}\right)$ abundance ratio could be fostered by the moderately low $N_{\mathrm{CO}}$ and the low temperature, which in turn are probably related to the high $n_{\mathrm{H} 2}$ volume density. We are pursuing an observational program to establish whether such a phenomenon is common in metal-poor starbursts.

\subsection{The $\mathrm{H}_{2}$ mass for NGC 1140 revealed with atomic carbon}

In Paper I, we derived the metallicity dependence for the COto- $\mathrm{H}_{2}$ conversion factor, $\alpha_{\mathrm{CO}} \propto\left(Z / Z_{\odot}\right)^{-2}$, which, at the metallicity of NGC 1140 , is roughly equivalent to the exponential variation with abundance found by Wolfire et al. (2010). Here we compare the molecular mass for NGC 1140 using $\alpha_{\mathrm{CO}}$ with the molecular mass estimated from $[\mathrm{CI}]$ luminosity according to Glover \& Clark (2016). After correcting to a $22^{\prime \prime}$ beam size as in Table 4 (see also Table A.1), $L^{\prime}(\mathrm{CO})=2.9 \times 10^{6} \mathrm{~K} \mathrm{~km} \mathrm{~s}^{-1} \mathrm{pc}^{2}$. Using $\alpha_{\mathrm{CO}}=3.2 M_{\odot}\left(\mathrm{K} \mathrm{km} \mathrm{s}^{-1} \mathrm{pc}^{2}\right)^{-1}$ (not including helium, Saintonge et al. 2011), and scaling with $\left(Z / Z_{\odot}\right)^{-2}$ (see Paper I) for $\mathrm{NGC} 1140\left(Z / Z_{\odot}=0.31 ; 12+\log (\mathrm{O} / \mathrm{H})=8.18\right.$, and assuming $12+\log (\mathrm{O} / \mathrm{H})_{\odot}=8.69$ from Asplund et al. 2009), we find a total molecular gas mass of $\sim 9.3 \times 10^{7} M_{\odot}$.

Glover \& Clark (2016) use their hydrodynamical simulations of $[\mathrm{CI}](1-0)$ emission to estimate $\mathrm{H}_{2}$ masses, and provide a [CI] luminosity-to- $\mathrm{H}_{2}$ conversion factor. At the highest $G_{0}$ modeled by Glover \& Clark (2016), they obtain a mean value $X_{\mathrm{CI}}=$ $8.8 \times 10^{20} \mathrm{~cm}^{-2}\left(\mathrm{~K} \mathrm{~km} \mathrm{~s}^{-1}\right)^{-1}$ for $Z \sim Z_{\odot}$ and an approximately linear increase with decreasing metallicity. For NGC 1140 the $[\mathrm{CI}](1-0)$ luminosity $L^{\prime}([\mathrm{CI}](1-0))=1.6 \times 10^{6} \mathrm{~K} \mathrm{~km} \mathrm{~s}^{-1} \mathrm{pc}^{2}$ (from Table 4, including the aperture correction for a $22^{\prime \prime}$ beam). Converting the Glover \& Clark (2016) $X_{\mathrm{CI}}$ to equivalent $\alpha_{\mathrm{CO}}$ units, we would find $\alpha_{\mathrm{CI}}=53.2 M_{\odot}\left(\mathrm{K} \mathrm{km} \mathrm{s}^{-1} \mathrm{pc}^{2}\right)^{-1}$ for $0.31 Z / Z_{\odot}$, and a resulting molecular mass $\mathrm{M}_{\mathrm{H} 2}$ of $\sim 8.7 \times$ $10^{7} M_{\odot}$. This value is within $10 \%$ of what we infer with the quadratic metallicity scaling of $\alpha_{\mathrm{CO}}$, despite the larger observed $[\mathrm{CI}](1-0) /{ }^{12} \mathrm{CO}(1-0)$ ratio (see Sect. 5.3) relative to their models ${ }^{8}$. These values of molecular gas mass for NGC 1140 are within $15 \%$ of those estimated using the models of Weiß et al. (2007).

\section{Summary and conclusions}

We have presented ${ }^{12} \mathrm{CO}$ observations of ten galaxies, with detections for eight. With metallicities ranging from $12+\log (\mathrm{O} / \mathrm{H}) \sim 7.7$ to $8.4\left(0.1 Z_{\odot}\right.$ to $\left.0.5 Z_{\odot}\right)$, at and below the abundance of the SMC, this is the largest sample of metal-poor galaxies with $\mathrm{CO}$ detections so far obtained outside the Local Group. For one of the galaxies, NGC 1140, we report additional ${ }^{13} \mathrm{CO},[\mathrm{CI}](1-0)$, and $\mathrm{HCN}(1-0)$ measurements. Our main conclusions are the following:

- After correcting for differences in beam sizes, NGC 1140 shows high velocity-integrated temperature ratios of ${ }^{12} \mathrm{CO}(2-1) /{ }^{12} \mathrm{CO}(1-0) \quad\left(R_{21}=2.1 \pm 0.06\right)$ and ${ }^{12} \mathrm{CO}(3-$ 2) $/{ }^{12} \mathrm{CO}(1-0)\left(R_{31}=2.0 \pm 0.45\right.$, or $R_{31}=1.7 \pm 0.39$ with the correction to $22^{\prime \prime}$ beams), while the other galaxies have less extreme ratios (see Figs. 3, 4).

8 In these calculations, unlike Paper I, we have not included the factor
of 1.64 to correct to total flux in NGC 1140; these values are corrected to $22^{\prime \prime}$ as in Tables 4 and A.1 
- A comparison of dense-gas tracers such as ${ }^{12} \mathrm{CO}(3-2)$ (of the four observed metal-poor galaxies) and $\mathrm{HCN}(1-0)$ (upper limit for NGC 1140) with data in the literature shows a deficit in ${ }^{12} \mathrm{CO}(3-2)$ relative to SFR (see Fig. 7), similar to the ${ }^{12} \mathrm{CO}(1-0)$ deficit found in Paper I. However, gas excitation at low metallicity measured by comparing ${ }^{12} \mathrm{CO}(3-2)$ to ${ }^{12} \mathrm{CO}(1-0)$ luminosities seems similar to that of more metal-rich systems. HCN(1-0) was observed but not detected in NGC 1140. The lower limit of HCN(10 ) for NGC 1140 may be due to a combination of stellar feedback (e.g., Hopkins et al. 2013) and an extremely high $[\mathrm{CO}] /[\mathrm{HCN}]$ abundance ratio $\left(\gtrsim 10^{5}\right)$ probably because of the low metallicity (see Fig. 15).

- Fitting LTE models to the ${ }^{12} \mathrm{CO}(3-2),{ }^{12} \mathrm{CO}(2-1)$, and ${ }^{12} \mathrm{CO}(1-0)$ emission in NGC 1140 , NGC 7077, and UM 448 suggests that $\mathrm{CO}$ column densities are moderate and temperatures are low. These fits also suggest low optical depths, but the lack of higher-J lines for NGC 7077 and UM 448 prevents confirming this as a general result with radiativetransfer models.

- Fitting physical models (RADEX) of the ${ }^{12} \mathrm{CO}$ and ${ }^{13} \mathrm{CO}$ emission in NGC 1140 suggests that the molecular gas is cool $\left(T_{\text {kin }} \lesssim 20 \mathrm{~K}\right)$, dense $\left(n_{\mathrm{H} 2} \gtrsim 10^{6} \mathrm{~cm}^{-3}\right)$, with moderate $\mathrm{CO}$ column density $\left(N_{\mathrm{CO}} \sim 10^{16} \mathrm{~cm}^{-2}\right)$ and low filling factor $(F \sim 0.01-0.1) .{ }^{12} \mathrm{CO}$ optical depths are fairly low $\left(\tau_{\mathrm{CO}} \lessgtr 0.2\right)$, and we speculate that the $\mathrm{CO}$ survives photodissociation because of the high HI column density in this galaxy. The molecular excitation in NGC 1140 is starburstlike, similar to M 82 (see Fig. 13).

- The $\left[{ }^{12} \mathrm{CO}\right] /\left[{ }^{13} \mathrm{CO}\right]$ abundance ratio in NGC 1140 inferred from the fit is very low, $\sim 8-20$, lower even than the value of $\sim 24$ found in the Galactic center (Langer \& Penzias 1990). Because the starburst in NGC 1140 is quite young, it is difficult to interpret this low ratio as due to stellar reprocessing by older intermediate-mass populations (e.g., Milam et al. 2005, 2009). Instead, we attribute it to enhanced CO fractionation, from the combination of moderate column densities and cool gas (see Fig. 16), as predicted by the chemical models of Röllig \& Ossenkopf (2013) and Szúcs et al. (2014).

- Fitting RADEX models of the ${ }^{12} \mathrm{CO}$ and $[\mathrm{CI}](1-0)$ emission in NGC 1140 gives physical conditions similar to the ${ }^{12} \mathrm{CO}+{ }^{13} \mathrm{CO}$ fits, although there is a possibility for warmer $\left(T_{\text {kin }} \sim 38 \mathrm{~K}\right)$, less dense $\left(n_{\mathrm{H} 2} \sim 10^{4} \mathrm{~cm}^{-2}\right)$ gas at higher column densities. Independent fitting results for NGC 1140 using the models of Weiß et al. (2007) give similar results. Both our RADEX fits and the LVG fits suggest that atomic carbon is at least 10 times more abundant than ${ }^{12} \mathrm{CO}$ in NGC 1140.

For the first time outside the Local Group, the six CO transitions measured for NGC 1140, together with [CI](1-0), have enabled an analysis of physical conditions in the molecular gas of a low-metallicity galaxy with at least 6 SSCs. The unusual $\left[{ }^{12} \mathrm{CO}\right] /\left[{ }^{13} \mathrm{CO}\right]$ and $\left[{ }^{12} \mathrm{CO}\right] /\left[{ }^{12} \mathrm{C}\right]$ abundance ratios, and the cool, dense gas at moderate column densities, may be the consequence of the SSCs in this galaxy and their feedback effect on the ISM. Future work will attempt to put this speculation on a more quantitative footing, both with models of ISM chemistry and with more observations of the molecular ISM in low-metallicity starbursts.

Acknowledgements. We acknowledge the anonymous referee for her/his useful comments and suggestions. L.K.H. is grateful to Carlo Giovanardi for interesting discussions and mathematical insights for the beam corrections. S.G.B. acknowledges economic support from grants ESP2015-68964-P and AYA201676682-C3-2-P. We warmly thank the IRAM staff, both in Granada and at Pico Veleta, for their capable management of the logistics and the telescope/receiver operations. We are also indebted to the APEX service observing team for their dedication in the challenging high-frequency observations obtained for NGC 1140. We gratefully acknowledge the International Space Science Institute (Bern) for hospitality during the conception of this paper. Heavy use was made of the NASA/IPAC Extragalactic Database (NED).

\section{References}

Albrecht, M., Chini, R., Krügel, E., Müller, S. A. H., \& Lemke, R. 2004, A\&A, 414, 141

Asplund, M., Grevesse, N., Sauval, A. J., \& Scott, P. 2009, ARA\&A, 47, 481

Barone, L. T., Heithausen, A., Hüttemeister, S., Fritz, T., \& Klein, U. 2000, MNRAS, 317, 649

Bayet, E., Gerin, M., Phillips, T. G., \& Contursi, A. 2006, A\&A, 460, 467

Bialy, S., \& Sternberg, A. 2015, MNRAS, 450, 4424

Bisbas, T. G., Papadopoulos, P. P., \& Viti, S. 2015, ApJ, 803, 37

Bisbas, T. G., van Dishoeck, E. F., Papadopoulos, P. P., et al. 2017, ApJ, 839, 90

Bolatto, A. D., Jackson, J. M., \& Ingalls, J. G. 1999, ApJ, 513, 275

Bolatto, A. D., Jackson, J. M., Kraemer, K. E., \& Zhang, X. 2000a, ApJ, 541, L17

Bolatto, A. D., Jackson, J. M., Israel, F. P., Zhang, X., \& Kim, S. 2000b, ApJ, 545,234

Bolatto, A. D., Jackson, J. M., Wilson, C. D., \& Moriarty-Schieven, G. 2000c, ApJ, 532, 909

Bolatto, A. D., Leroy, A. K., Rosolowsky, E., Walter, F., \& Blitz, L. 2008, ApJ, 686, 948

Bolatto, A. D., Wolfire, M., \& Leroy, A. K. 2013, ARA\&A, 51, 207

Boothroyd, A. I., \& Sackmann, I.-J. 1999, ApJ, 510, 232

Braine, J., \& Combes, F. 1992, A\&A, 264, 433

Buchbender, C., Kramer, C., Gonzalez-Garcia, M., et al. 2013, A\&A, 549, A17

Buyle, P., Michielsen, D., de Rijcke, S., Ott, J., \& Dejonghe, H. 2006, MNRAS, 373, 793

Carilli, C. L., \& Walter, F. 2013, ARA\&A, 51, 105

Cohen, R. S., Dame, T. M., Garay, G., et al. 1988, ApJ, 331, L95

Cormier, D., Lebouteiller, V., Madden, S. C., et al. 2012, A\&A, 548, A20

Cormier, D., Madden, S. C., Lebouteiller, V., et al. 2014, A\&A, 564, A121

de Grijs, R., Smith, L. J., Bunker, A., et al. 2004, MNRAS, 352, 263

Draine, B. T. 1978, ApJS, 36, 595

Draine, B. T., Dale, D. A., Bendo, G., et al. 2007, ApJ, 663, 866

Elmegreen, B. G., Rubio, M., Hunter, D. A., et al. 2013, Nature, 495, 487

Fukui, Y., Mizuno, N., Yamaguchi, R., et al. 1999, PASJ, 51, 745

Fumagalli, M., Krumholz, M. R., \& Hunt, L. K. 2010, ApJ, 722, 919

Gao, Y., \& Solomon, P. M. 2004, ApJ, 606, 271

García-Burillo, S., Usero, A., Alonso-Herrero, A., et al. 2012, A\&A, 539, A8

Gerin, M., \& Phillips, T. G. 2000, ApJ, 537, 644

Glover, S. C. O., \& Clark, P. C. 2016, MNRAS, 456, 3596

Goldsmith, P. F., \& Langer, W. D. 1999, ApJ, 517, 209

Gondhalekar, P. M., Johansson, L. E. B., Brosch, N., Glass, I. S., \& Brinks, E. 1998, A\&A, 335, 152

Graciá-Carpio, J., García-Burillo, S., Planesas, P., Fuente, A., \& Usero, A. 2008, A\&A, 479, 703

Greve, T. R., Leonidaki, I., Xilouris, E. M., et al. 2014, ApJ, 794, 142

Henkel, C., Asiri, H., Ao, Y., et al. 2014, A\&A, 565, A3

Hollenbach, D. J., Takahashi, T., \& Tielens, A. G. G. M. 1991, ApJ, 377, 192

Hopkins, P. F., Narayanan, D., Murray, N., \& Quataert, E. 2013, MNRAS, 433, 69

Hunt, L. K., Thuan, T. X., Izotov, Y. I., \& Sauvage, M. 2010, ApJ, 712, 164

Hunt, L. K., García-Burillo, S., Casasola, V., et al. 2015, A\&A, 583, A114 (Paper I)

Hunter, D. A., van Woerden, H. \& Gallagher, J. S., III 1994, ApJS, 91, 79

Indebetouw, R., Brogan, C., Chen, C.-H. R., et al. 2013, ApJ, 774, 73

Israel, F. P. 2005, A\&A, 438, 855

Israel, F. P. 2009, A\&A, 493, 525

Israel, F. P., \& Baas, F. 2002, A\&A, 383, 82

Israel, F. P., \& Baas, F. 2003, A\&A, 404, 495

Israel, F. P., Baas, F., Rudy, R. J., Skillman, E. D., \& Woodward, C. E. 2003, A\&A, 397, 87

Israel, F. P., Rosenberg, M. J. F., \& van der Werf, P. 2015, A\&A, 578, A95

Johnson, K. E., Leitherer, C., Vacca, W. D., \& Conti, P. S. 2000, AJ, 120, 1273

Kepley, A. A., Leroy, A. K., Johnson, K. E., Sandstrom, K., \& Chen, C.-H. R. 2016, ApJ, 828, 50

Kobulnicky, H. A., Kennicutt, R. C., Jr., \& Pizagno, J. L. 1999, ApJ, 514, 544

Kramer, C., Jakob, H., Mookerjea, B., et al. 2004, A\&A, 424, 887 
Krips, M., Martín, S., Sakamoto, K., et al. 2016, A\&A, 592, L3

Kuno, N., Sato, N., Nakanishi, H., et al. 2007, PASJ, 59, 117

Lada, C. J., Forbrich, J., Lombardi, M., \& Alves, J. F. 2012, ApJ, 745, 190

Langer, W. D., \& Penzias, A. A. 1990, ApJ, 357, 477

Lequeux, J., Le Bourlot, J., Pineau des Forets, G., et al. 1994, A\&A, 292, 371

Leroy, A., Bolatto, A. D., Simon, J. D., \& Blitz, L. 2005, ApJ, 625, 763

Leroy, A., Cannon, J., Walter, F., Bolatto, A., \& Weiß, A. 2007, ApJ, 663, 990

Leroy, A. K., Walter, F., Bigiel, F., et al. 2009, AJ, 137, 4670

Liszt, H., \& Lucas, R. 2001, A\&A, 370, 576

Liu, L., Weiß, A., Perez-Beaupuits, J. P., et al. 2017, ApJ, 846, 5

Mao, R.-Q., Schulz, A., Henkel, C., et al. 2010, ApJ, 724, 1336

Mauersberger, R., Henkel, C., Walsh, W., \& Schulz, A. 1999, A\&A, 341, 256

Meier, D. S., Turner, J. L., Crosthwaite, L. P., \& Beck, S. C. 2001, AJ, 121, 740

Meijerink, R., Spaans, M., \& Israel, F. P. 2007, A\&A, 461, 793

Milam, S. N., Savage, C., Brewster, M. A., Ziurys, L. M., \& Wyckoff, S. 2005, ApJ, 634, 1126

Milam, S. N., Woolf, N. J., \& Ziurys, L. M. 2009, ApJ, 690, 837

Minamidani, T., Mizuno, N., Mizuno, Y., et al. 2008, ApJS, 175, 485

Moll, S. L., Mengel, S., de Grijs, R., Smith, L. J., \& Crowther, P. A. 2007 MNRAS, 382, 1877

Mühle, S., Seaquist, E. R., \& Henkel, C. 2007, ApJ, 671, 1579

Narayanan, D., Groppi, C. E., Kulesa, C. A., \& Walker, C. K. 2005, ApJ, 630, 269

Narayanan, D., Cox, T. J., Shirley, Y., et al. 2008, ApJ, 684, 996

Nikolić, S., Garay, G., Rubio, M., \& Johansson, L. E. B. 2007, A\&A, 471, 561

Oka, T., Nagai, M., Kamegai, K., Tanaka, K., \& Kuboi, N. 2007, PASJ, 59, 15

Okada, Y., Requena-Torres, M. A., Güsten, R., et al. 2015, A\&A, 580, A54

Ott, J., Henkel, C., Staveley-Smith, L., \& Weiß, A. 2010, ApJ, 710, 105

Papadopoulos, P. P., Thi, W.-F., \& Viti, S. 2004, MNRAS, 351, 147

Papadopoulos, P. P., van der Werf, P. P., Xilouris, E. M., et al. 2012, MNRAS, 426, 2601

Paron, S., Ortega, M. E., Cunningham, M., et al. 2014, A\&A, 572, A56

Paron, S., Ortega, M. E., Fariña, C., et al. 2016, MNRAS, 455, 518

Pavlenko, Y. V., Jones, H. R. A., \& Longmore, A. J. 2003, MNRAS, 345, 311

Pineda, J. L., Mizuno, N., Röllig, M., et al. 2012, A\&A, 544, A84

Pineda, J. L., Langer, W. D., Goldsmith, P. F., et al. 2017, ApJ, 839, 107

Rémy-Ruyer, A., Madden, S. C., Galliano, F., et al. 2013, A\&A, 557, A95

Rémy-Ruyer, A., Madden, S. C., Galliano, F., et al. 2014, A\&A, 563, A31
Requena-Torres, M. A., Israel, F. P., Okada, Y., et al. 2016, A\&A, 589, A28 Röllig, M., \& Ossenkopf, V. 2013, A\&A, 550, A56

Rothman, L. S., Gordon, I. E., Barbe, A., et al. 2009, J. Quant. Spectr. Rad. Transf., 110, 533

Rubio, M., Lequeux, J., \& Boulanger, F. 1993, A\&A, 271, 9

Rubio, M., Elmegreen, B. G., Hunter, D. A., et al. 2015, Nature, 525, 218

Sage, L. J., Salzer, J. J., Loose, H.-H., \& Henkel, C. 1992, A\&A, 265, 19

Saintonge, A., Kauffmann, G., Wang, J., et al. 2011, MNRAS, 415, 61

Sakamoto, S. 1999, ApJ, 523, 701

Santangelo, G., Testi, L., Gregorini, L., et al. 2009, A\&A, 501, 495

Sato, M. \& Lee, I. C. 1983, J. Chem. Phys., 78, 4515

Schöier, F. L., van der Tak, F. F. S., van Dishoeck, E. F., \& Black, J. H. 2005, A\&A, 432, 369

Schruba, A., Leroy, A. K., Walter, F., et al. 2012, AJ, 143, 138

Schruba, A., Leroy, A. K., Kruijssen, J. M. D., et al. 2017, ApJ, 835, 278

Shi, Y., Wang, J., Zhang, Z.-Y., et al. 2015, ApJ, 804, L11

Shi, Y., Wang, J., Zhang, Z.-Y., et al. 2016, Nat. Commun., 7, 13789

Szúcs, L., Glover, S. C. O., \& Klessen, R. S. 2014, MNRAS, 445, 4055

Tang, X. D., Henkel, C., Chen, C.-H. R., et al. 2017, A\&A, 600, A16

Taylor, C. L., Kobulnicky, H. A., \& Skillman, E. D. 1998, AJ, 116, 2746

van der Tak, F. F. S., Black, J. H., Schöier, F. L., Jansen, D. J., \& van Dishoeck, E. F. 2007, A\&A, 468, 627

Viti, S., García-Burillo, S., Fuente, A., et al. 2014, A\&A, 570, A28

Walter, F., Weiß, A., Downes, D., Decarli, R., \& Henkel, C. 2011, ApJ, 730, 18

Wang, M., Chin, Y.-N., Henkel, C., Whiteoak, J. B., \& Cunningham, M. 2009, ApJ, 690, 580

Watson, W. D., Anicich, V. G., \& Huntress, W. T., Jr. 1976, ApJ, 205, L165

Weiß, A., Neininger, N., Henkel, C., Stutzki, J., \& Klein, U. 2001, ApJ, 554, L143

Weiß, A., Walter, F., \& Scoville, N. Z. 2005, A\&A, 438, 533

Weiß, A., Downes, D., Neri, R., et al. 2007, A\&A, 467, 955

Wolfire, M. G., Hollenbach, D., \& McKee, C. F. 2010, ApJ, 716, 1191

Wong, T., Hughes, A., Fukui, Y., et al. 2009, ApJ, 696, 370

Wu, J., Evans, N. J., II, Shirley, Y. L., \& Knez, C. 2010, ApJS, 188, 313

Young, J. S., Xie, S., Tacconi, L., et al. 1995, ApJS, 98, 219

Zhang, J. S., Henkel, C., Mauersberger, R., et al. 2007, A\&A, 465, 887

Zhu, M., Papadopoulos, P. P., Xilouris, E. M., Kuno, N., \& Lisenfeld, U. 2009, ApJ, 706, 941 


\section{Appendix A: Exponential beam size correction}

Following Liu et al. (2017), we have corrected the velocityintegrated $T_{\mathrm{mb}}$ values to a common beam size according to the distribution of cool dust, thus explicitly assuming that the molecular gas and the dust have the same distribution. In order to apply such a correction also to the two galaxies (Mrk 0996, NGC 7077) for which this was not possible, we have adopted an analytical approach; such an approach also tends to average out photometric uncertainties and possibly improves the reliability of the correction.

PACS $160 \mu \mathrm{m}$ images are available from the Herschel archive for NGC 1140, NGC 3353, and UM 448, but for NGC 1156, we had to use the MIPS $160 \mu \mathrm{m}$ image from Spitzer. In all cases, the $160 \mu \mathrm{m}$ PACS fluxes reported by Rémy-Ruyer et al. (2013) are beyond the peak of the dust emission, implying that in these dwarf galaxies the $160 \mu \mathrm{m}$ is tracing cool dust. We obtained surface brightness profiles by performing an azimuthal extraction centered on the brightness peak, and growth curves by measuring photometric flux in increasingly large circular apertures. The $160 \mu \mathrm{m}$ surface brightness profiles are shown in the left panels and the growth curves in the right panels of Figs. A.1-A.4. The dust distribution is clearly exponential (rather than Gaussian) in all cases examined. For the two remaining galaxies, Mrk 996 and NGC 7077, MIPS $160 \mu \mathrm{m}$ images are available, but the galaxies are not resolved (even at 24 or $70 \mu \mathrm{m}$ where we obtained clear profiles of a diffraction-limited Airy ring).

Taking the source distribution to be exponential, with folding length $r_{\mathrm{s}}$, the integrated flux density within a beam with FWHM $\theta_{\mathrm{b}}$ corresponds to the following integral (assuming azimuthal symmetry) in polar coordinates:

$S_{v}=A_{0} \int_{0}^{2 \pi} \int_{0}^{\theta_{\mathrm{b}} / 2} \exp \left(-r / r_{\mathrm{s}}\right) r \mathrm{~d} r \mathrm{~d} \phi$

where $A_{0}$ is the brightness at the origin of the exponential, and $r$ corresponds to the angular distance from the origin. Eq. (A.1) can be solved analytically to obtain:

$S_{v}=2 \pi A_{0}\left\{r_{\mathrm{s}}^{2}\left[1-\exp \left(-\frac{\theta_{\mathrm{b}}}{2 r_{\mathrm{s}}}\right)\right]-\frac{\theta_{\mathrm{b}} r_{\mathrm{s}}}{2} \exp \left(-\frac{\theta_{\mathrm{b}}}{2 r_{\mathrm{s}}}\right)\right\}$.

The source FWHM $\theta_{\mathrm{s}}$ is related to the exponential folding length by $\theta_{\mathrm{s}}=2 r_{\mathrm{s}} \ln (2)$, obtained by calculating the diameter (i.e., twice the radius) at which the exponential distribution is equal to $A_{0} / 2$.

However, Eq. (A.1) is formally incorrect because of the need to convolve the pure exponential with the Gaussian beam when the exponential scale length $r_{\mathrm{s}}$ is small relative to the beam radius $\theta_{\mathrm{b}} / 2$. This convolution is given by the following, where we have taken advantage of the azimuthal symmetry and calculate the convolution along a single radial ray here given by $x$ :

$D(x, 0)=A_{0} \mathrm{e}^{-x^{2} / \sigma_{\mathrm{b}}^{2}} \int_{0}^{\infty} \int_{0}^{2 \pi} \mathrm{e}^{-\frac{r_{0}}{r_{\mathrm{s}}}} \mathrm{e}^{\left(-\frac{r_{0}^{2}-2 x r_{0} \cos \phi}{\sigma_{\mathrm{b}}^{2}}\right)} r_{0} \mathrm{~d} r_{0} \mathrm{~d} \phi$

where $\sigma_{\mathrm{b}}$ corresponds to the Gaussian $\sigma$ assuming that the beam is Gaussian with FWHM $\theta_{\mathrm{b}}\left(\theta_{\mathrm{b}}=2 \sigma_{\mathrm{b}} \sqrt{2 \ln 2}\right)$.

Equation (A.3) can be integrated by separating the variables $\left(r_{0}, \phi\right)$. After integrating in $\phi$, we obtain:

$D(x, 0)=2 \pi A_{0} \mathrm{e}^{-x^{2} / \sigma_{\mathrm{b}}^{2}} \int_{0}^{\infty} \mathrm{e}^{-\frac{r_{0}}{r_{\mathrm{s}}}} \mathrm{e}^{-\frac{r_{0}^{2}}{\sigma_{\mathrm{b}}^{2}}} I_{0}\left(\frac{2 x r_{0}}{\sigma_{\mathrm{b}}^{2}}\right) r_{0} \mathrm{~d} r_{0}$,
Table A.1. Flux corrections to a common beam size.

\begin{tabular}{|c|c|c|c|c|c|c|c|}
\hline Galaxy & $\begin{array}{c}r_{\mathrm{s}} \\
(\operatorname{arcsec})\end{array}$ & Transition & $\begin{array}{l}\text { Original beam } \\
\quad(\operatorname{arcsec})\end{array}$ & $\begin{array}{l}\text { Common beam } \\
\quad(\operatorname{arcsec})\end{array}$ & $\begin{array}{c}\text { Aperture } \\
\text { correction } \\
A P_{\text {cor }}\end{array}$ & $\begin{array}{c}T_{\mathrm{mb}} \\
\text { correction }\end{array}$ & $\begin{array}{c}I_{\mathrm{CO}} \\
\text { corrected } \\
\left(\mathrm{K} \mathrm{km} \mathrm{s}^{-1}\right)\end{array}$ \\
\hline Mrk 0996 & 3.6 & ${ }^{12} \mathrm{CO}(1-0)$ & 21.4 & 22.0 & 1.014 & 0.964 & 0.235 \\
\hline Mrk 0996 & 3.6 & ${ }^{12} \mathrm{CO}(2-1)($ IRAM) & 10.7 & 22.0 & 1.843 & 0.438 & 0.128 \\
\hline Mrk 0996 & 3.6 & ${ }^{12} \mathrm{CO}(2-1)(\mathrm{APEX})$ & 27.2 & 22.0 & 0.908 & 1.389 & 0.938 \\
\hline Mrk 0996 & 3.6 & ${ }^{12} \mathrm{CO}(3-2)$ & 18.1 & 22.0 & 1.13 & 0.77 & $<0.161$ \\
\hline NGC 1140 & 6.4 & ${ }^{12} \mathrm{CO}(1-0)$ & 21.4 & 22.0 & 1.027 & 0.976 & 0.564 \\
\hline NGC 1140 & 6.4 & ${ }^{12} \mathrm{CO}(2-1)$ & 10.7 & 22.0 & 2.502 & 0.595 & 1.180 \\
\hline NGC 1140 & 6.4 & ${ }^{12} \mathrm{CO}(3-2)$ & 18.1 & 22.0 & 1.238 & 0.841 & 0.972 \\
\hline NGC 1140 & 6.4 & ${ }^{12} \mathrm{CO}(4-3)$ & 13.6 & 22.0 & 1.784 & 0.682 & 0.582 \\
\hline NGC 1140 & 6.4 & ${ }^{13} \mathrm{CO}(1-0)$ & 22.4 & 22.0 & 0.980 & 1.019 & 0.053 \\
\hline NGC 1140 & 6.4 & ${ }^{13} \mathrm{CO}(2-1)$ & 11.2 & 22.0 & 2.342 & 0.609 & 0.105 \\
\hline NGC 1140 & 6.4 & {$[\mathrm{CI}](1-0)$} & 12.7 & 22.0 & 1.952 & 0.655 & 0.369 \\
\hline NGC 1156 & 44.9 & ${ }^{12} \mathrm{CO}(1-0)$ & 21.4 & 22.0 & 1.055 & 0.995 & 1.841 \\
\hline NGC 1156 & 44.9 & ${ }^{12} \mathrm{CO}(2-1)$ & 10.7 & 22.0 & 3.903 & 0.921 & 0.668 \\
\hline NGC 3353 & 5.4 & ${ }^{12} \mathrm{CO}(1-0)$ & 21.4 & 22.0 & 1.025 & 0.971 & 2.100 \\
\hline NGC 3353 & 5.4 & ${ }^{12} \mathrm{CO}(2-1)$ & 10.7 & 22.0 & 2.314 & 0.548 & 1.876 \\
\hline NGC 7077 & 4.8 & ${ }^{12} \mathrm{CO}(1-($ & 21.4 & 22.0 & 1.022 & 0.969 & 0.474 \\
\hline NGC 7077 & 4.8 & ${ }^{12} \mathrm{CO}(2-1)$ & 10.7 & 22.0 & 2.175 & 0.516 & 0.548 \\
\hline NGC 7077 & 4.8 & ${ }^{12} \mathrm{CO}(3-2)$ & 18.1 & 22.0 & 1.186 & 0.804 & 0.431 \\
\hline UM 448 & 4.4 & ${ }^{12} \mathrm{CO}(1-0)$ & 21.4 & 22.0 & 1.009 & 0.985 & 1.035 \\
\hline UM 448 & 4.4 & ${ }^{12} \mathrm{CO}(2-1)$ (IRAM) & 10.7 & 22.0 & 2.036 & 0.497 & 1.237 \\
\hline UM 448 & 4.4 & ${ }^{12} \mathrm{CO}(2-1)(\mathrm{APEX})$ & 27.6 & 22.0 & 0.869 & 1.365 & 2.106 \\
\hline UM 448 & 4.4 & ${ }^{12} \mathrm{CO}(3-2)$ & 18.1 & 22.0 & 1.154 & 0.806 & 0.705 \\
\hline
\end{tabular}

Notes. Assuming that the $\mathrm{CO}$ is distributed in an exponential disk like the dust.

where $I_{0}$ is a modified Bessel function of the first kind. Eq. (A.4) can then be integrated numerically to obtain the surface brightness profile of the convolved exponential, as shown as the lower (red) dashed curves in Figs. A.1-A.4.

These figures show that for the four galaxies for which we were able to fit surface-brightness profiles and growth curves the pure exponential $\left(2 \pi A_{0} \exp \left(-r / r_{\mathrm{s}}\right)\right)$ is equivalent to the integral given in Eq. (A.4) as long as the scale length $r_{\mathrm{s}}$ is larger than $\theta_{\mathrm{b}} / 2$. We thus fitted the growth curves with Eq. (A.2) in order to obtain the beam corrections. This is advantageous from a numerical point of view because otherwise it would be necessary to fit the growth curve to the integral of Eq. (A.4) which is in itself an integral.

To correct the fluxes (e.g., $S_{v}$ in Jy), we scaled the observed dust fluxes to a common beam size of $22^{\prime \prime}$ by comparing the growth-curve integrals in the different beam sizes using the best-fit of the analytical formula in Eq. (A.2) to obtain $r_{\mathrm{s}}$ and $A_{0}$. We thus derive a multiplicative factor $A P_{\text {cor }}$ as given in Col. (6) of Table A.1: $S_{22}=S_{\text {orig }} A P_{\text {cor }}$. We then apply the correction to velocity-integrated $T_{\mathrm{mb}}$ values by noting that $S / \Omega=2 k v^{2} / c^{2} T_{\mathrm{mb}}$, where $\Omega$ is the beam solid angle, $k$ is the Boltzmann constant, $v$ is the observed frequency, and $c$ is the speed of light. Thus:

$T_{\mathrm{mb}}(22)=T_{\mathrm{mb}}$ (orig) $A P_{\text {cor }} \frac{\Omega \text { (orig) }}{\Omega(22)}$

where "orig" refers to the original values observed in the different beams. The flux and $T_{\mathrm{mb}}$ corrections necessary to convert our observations to a common beam size of $22^{\prime \prime}$ are given in Table A.1, together with the corrected velocity-integrated $T_{\mathrm{mb}}$ values that we use throughout the paper when we are examining line ratios $\left({ }^{12} \mathrm{CO}(2-1) /{ }^{12} \mathrm{CO}(1-0)\right)$ or fitting physical models. Corrected values are also reported in Tables $2-4$ in the main text.

For Mrk 996 and NGC 7077, the only available cool dust images are from MIPS and, as mentioned above, the galaxies are not resolved. Thus, for these we assumed that the disk scale length $r_{\mathrm{s}}$ is equal to $0.2 R_{\mathrm{opt}}$ (optical radius from NED), consistently with Young et al. (1995), Leroy et al. (2009), Kuno et al. (2007) who all found that CO exponential scale lengths are, in the mean, equal to $1 / 5$ the optical radius. This is also roughly consistent with the other four galaxies for which the mean $r_{\mathrm{s}} / R_{\mathrm{opt}}$ given by the best fits of the growth curves is $0.27 \pm 0.16$. 
L. K. Hunt et al.: Physical conditions at low metallicity
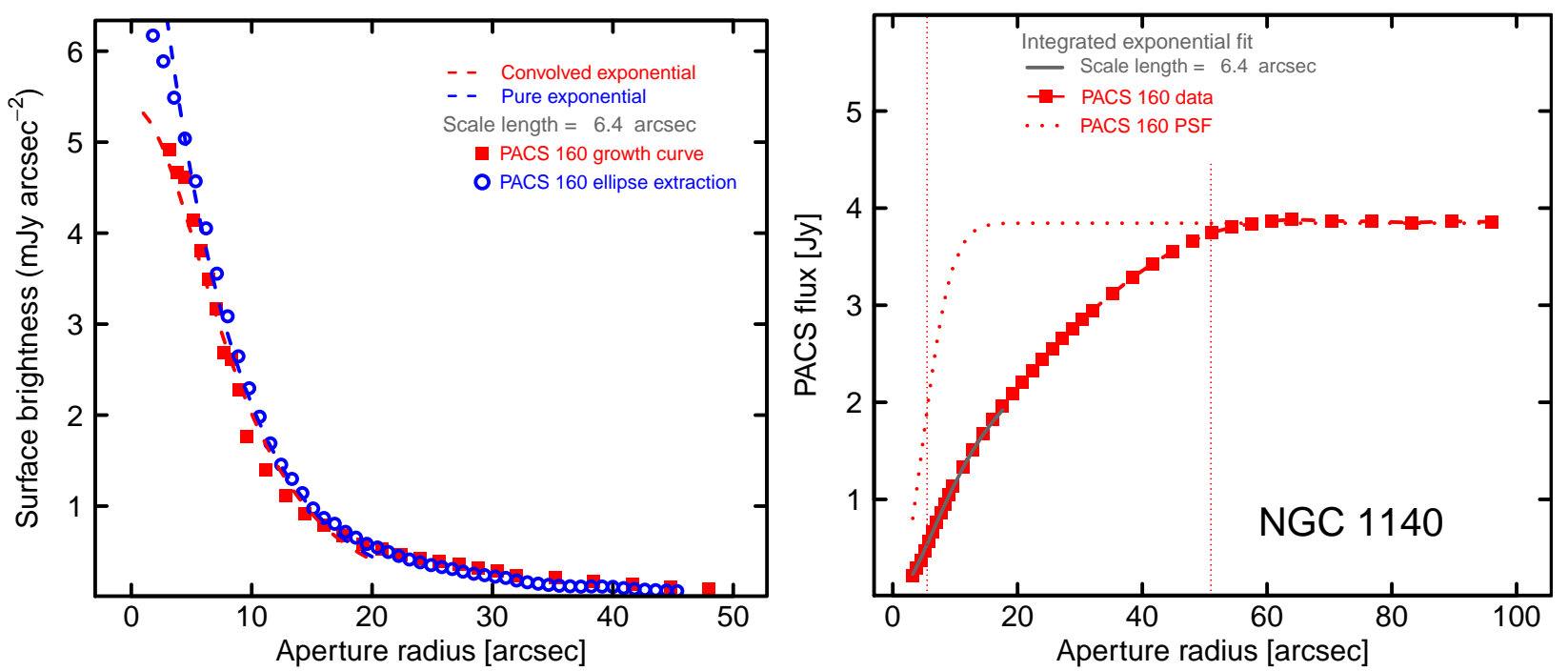

Fig. A.1. Left panel: PACS $160 \mu \mathrm{m}$ radial surface brightness profile of NGC 1140; open (blue) circles give the profile obtained from azimuthally averaging the image, and filled (red) squares the analogous profile obtained from the first derivative of the photometric growth curve. The lower dashed curve show an exponential distribution of scale length 6".4 convolved with the PACS $160 \mu \mathrm{m}$ beam (assuming it is Gaussian), and the upper one the same exponential but without convolution. Right panel: PACS $160 \mu \mathrm{m}$ growth curve centered on the brightness peak. The left-most dotted vertical line gives the PACS beam radius $\left(\sim 5^{\prime \prime} 5\right)$ and the right-most one the optical radius of NGC 1140 taken from NED (51"). The solid grey curve corresponds to the best-fit exponential integral as described in the text.
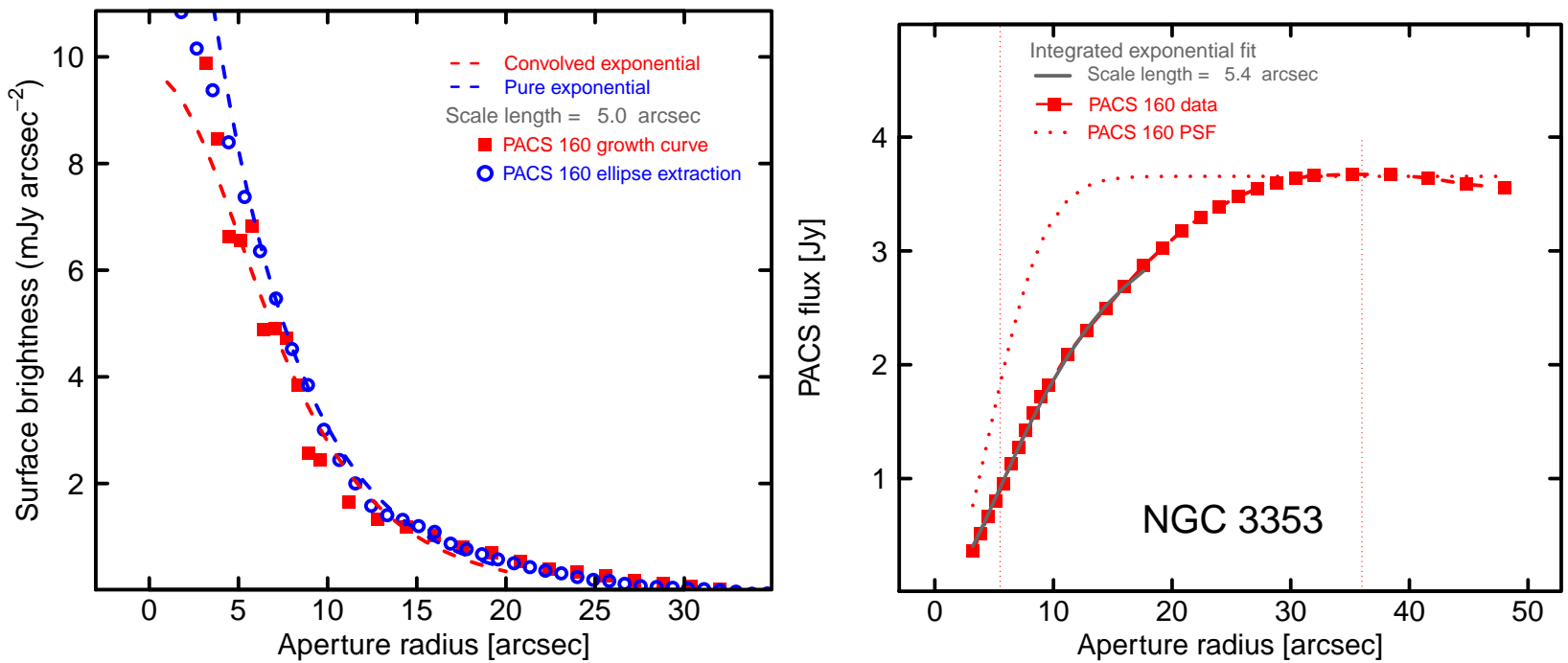

Fig. A.2. Left panel: PACS $160 \mu \mathrm{m}$ radial surface brightness profile of NGC 3353 (Haro 3); open (blue) circles give the profile obtained from averaging the image over fixed-position-angle ellipses, and filled (red) squares the analogous profile obtained from the first derivative of the photometric growth curve. As in Fig. A.1, the dashed curves show the exponential convolved with the PACS $160 \mu \mathrm{m}$ beam (lower), and the upper the exponential without convolution; the best-fit scale length is 5'”0. Right panel: PACS $160 \mu \mathrm{m}$ growth curve centered on the brightness peak. The left-most dotted vertical line gives the PACS beam radius $\left(\sim 5^{\prime \prime} .5\right)$ and the right-most one the optical radius of NGC 3353 taken from NED (36"). The solid grey curve corresponds to the best-fit exponential integral as described in the text; in the case of NGC 3353, the growth-curve best fit scalelength is slightly different from the radial profile one and we have adopted the growth-curve value for beam corrections. 

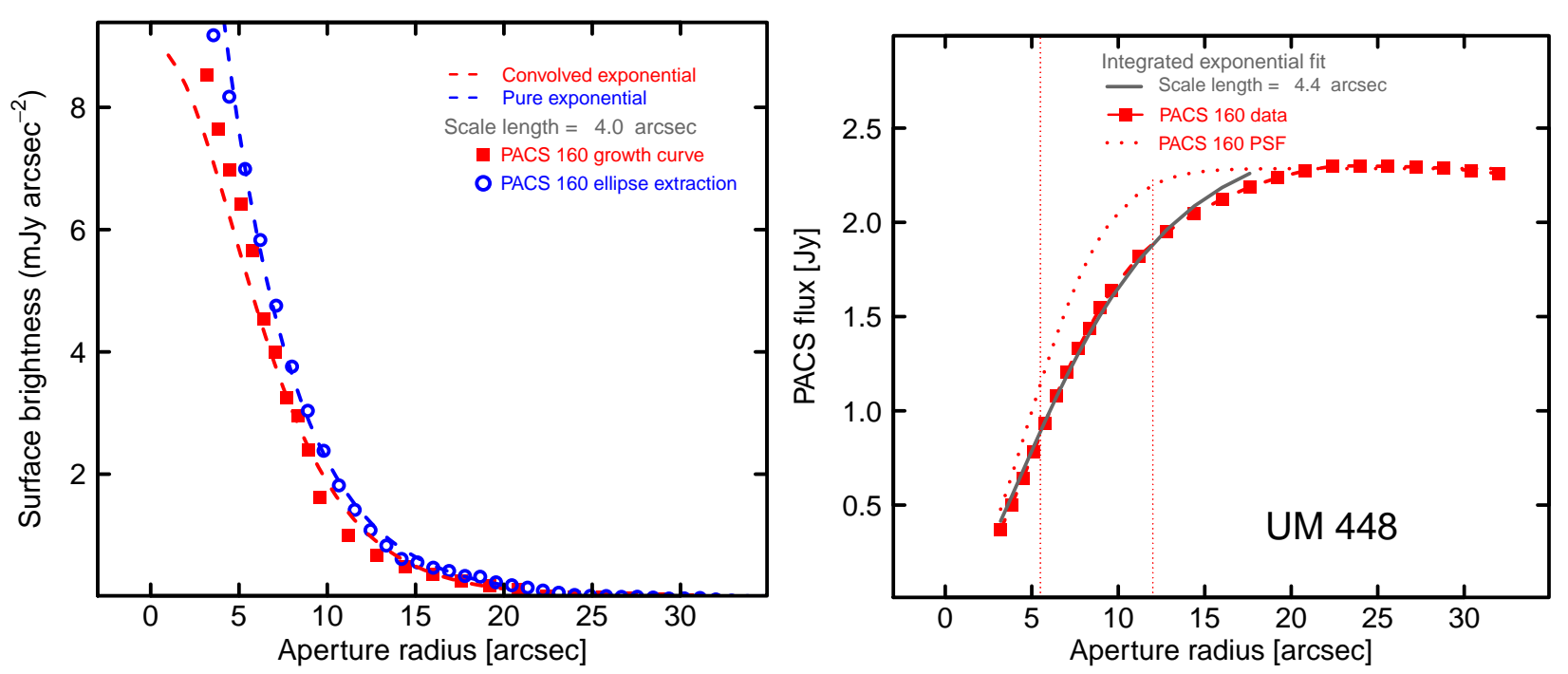

Fig. A.3. Left panel: PACS $160 \mu \mathrm{m}$ radial surface brightness profile of UM 448; open (blue) circles give the profile obtained from averaging the image over fixed-position-angle ellipses, and filled (red) squares the analogous profile obtained from the first derivative of the photometric growth curve. As in Fig. A.1, the dashed curves show the exponential convolved with the PACS $160 \mu \mathrm{m}$ beam (lower), and the upper the exponential without convolution; the best-fit scale length is 4".0. Right panel: PACS $160 \mu \mathrm{m}$ growth curve centered on the brightness peak. The left-most dotted vertical line gives the PACS beam radius $\left(\sim 5^{\prime \prime} 5\right)$ and the right-most one the optical radius of UM 448 taken from NED $\left(12^{\prime \prime}\right)$. The solid grey curve corresponds to the best-fit exponential integral as described in the text; like NGC 3353, the growth-curve best fit scalelength for UM 448 is slightly different from the radial profile one and we have adopted the growth-curve value for beam corrections.
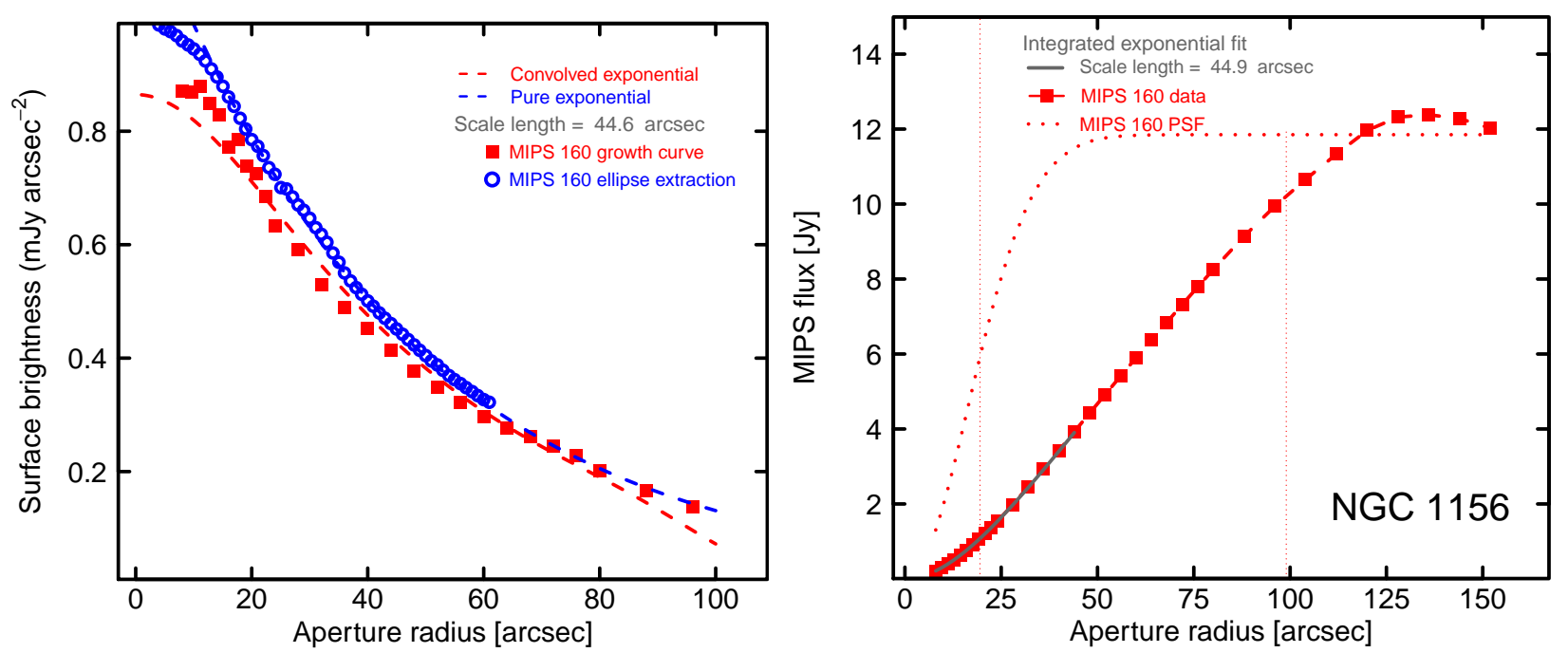

Fig. A.4. Left panel: MIPS $160 \mu \mathrm{m}$ radial surface brightness profile of NGC 1156; open (blue) circles give the profile obtained from averaging the image over fixed-position-angle ellipses, and filled (red) squares the analogous profile obtained from the first derivative of the photometric growth curve. As in Fig. A.1, the dashed curves show the exponential convolved with the MIPS $160 \mu \mathrm{m}$ beam (lower), and the upper the exponential without convolution; the best-fit scale length is 4".0. Right panel: MIPS $160 \mu \mathrm{m}$ growth curve centered on the brightness peak. The left-most dotted vertical line gives the MIPS beam radius $\left(\sim 19^{\prime \prime}\right)$ and the right-most one the optical radius of NGC 1156 taken from NED (99"). The solid grey curve corresponds to the best-fit exponential integral as described in the text; like NGC 3353 and UM 448, the growth-curve best fit scalelength for NGC 1156 is slightly different from the radial profile one and we have adopted the growth-curve value for beam corrections. 


\section{Appendix B: Gaussian beam size correction}

To ensure that our results do not depend on the specific formulation of the correction for beam dilution, here we investigate a different assumption for the distribution of the $\mathrm{CO}$ emission, namely a Gaussian. For a Gaussian source distribution with a FWHM $\theta_{\mathrm{s}}$ (corresponding to a Gaussian $\sigma_{\mathrm{s}}=\theta_{\mathrm{s}} /[2 \sqrt{2 \ln (2)}]$ ), the integrated flux density within a beam with FWHM $\theta_{\mathrm{b}}$ is given by the following integral (assuming azimuthal symmetry) in polar coordinates:

$S_{v}=A_{0} \int_{0}^{2 \pi} \int_{0}^{\theta_{\mathrm{b}} / 2} \exp \left(\frac{-r}{2 \sigma_{\mathrm{s}}^{2}}\right) r \mathrm{~d} r \mathrm{~d} \phi$

where $A_{0}$ is the normalization constant, and $r$ corresponds to the angular distance from the origin. Equation (B.1) can be solved analytically to obtain:

$S_{v}=2 \pi A_{0} \sigma_{\mathrm{s}}^{2}\left[1-\exp \left(-\frac{\theta_{\mathrm{b}}^{2}}{8 \sigma_{\mathrm{s}}^{2}}\right)\right]$.

However, like Eq. (A.1), Eq. (B.1) is formally incorrect because of the need to convolve the source Gaussian distribution with the Gaussian beam. This convolution is given by the following, where we have taken advantage of the azimuthal symmetry and calculate the convolution in Cartesian coordinates:

$D(x, y)=A_{0} \int_{-\infty}^{\infty} \int_{-\infty}^{\infty} \mathrm{e}^{-\frac{-\left(x_{0}^{2}+y_{0}^{2}\right)}{\sigma_{\mathrm{s}}^{2}}} \mathrm{e}^{-\frac{-\left(x-x_{0}\right)^{2}-\left(y-y_{0}\right)^{2}}{\sigma_{\mathrm{b}}^{2}}} \mathrm{~d} x_{0} \mathrm{~d} y_{0}$

where $\sigma_{\mathrm{b}}$ corresponds to the Gaussian $\sigma$ assuming that the beam is Gaussian with FWHM $\theta_{\mathrm{b}}\left(=2 \sigma_{\mathrm{b}} \sqrt{2 \ln 2}\right)$.

It is straightforward to integrate Eq. (B.3) analytically to obtain:

$D(r)=A_{0}^{\prime} \frac{\sigma_{\mathrm{s}}^{2}}{\left(\sigma_{\mathrm{b}}^{2}+\sigma_{\mathrm{s}}^{2}\right)} \exp \left[\frac{-r^{2}}{\left(\sigma_{\mathrm{b}}^{2}+\sigma_{\mathrm{s}}^{2}\right)}\right]$

where $A_{0}$ has been substituted by the inclusion of the normalization constant of a two-dimensional Gaussian with integral unity, $A_{0}=A_{0}^{\prime} /\left(\pi \sigma_{\mathrm{b}}^{2}\right)$; the independent Cartesian coordinates $x$ and $y$ have now been substituted with $r=\sqrt{x^{2}+y^{2}}$. Unlike Eq. (A.4) for the exponential source distribution, Eq. (B.4) can be easily
Table B.1. Flux corrections to a common beam size.

\begin{tabular}{ccccccc}
\hline \hline Galaxy & Transition & $\begin{array}{c}\text { Original beam } \\
\text { (arcsec) }\end{array}$ & $\begin{array}{c}\text { Common beam } \\
\text { (arcsec) }\end{array}$ & $\begin{array}{c}\text { Aperture } \\
\text { correction } \\
A P_{\text {cor }}\end{array}$ & $\begin{array}{c}T_{\mathrm{mb}} \\
\text { correction }\end{array}$ & $\begin{array}{c}I_{\mathrm{CO}} \\
\text { corrected } \\
\left(\mathrm{K} \mathrm{km} \mathrm{s}^{-1}\right)\end{array}$ \\
\hline NGC 1140 & ${ }^{12} \mathrm{CO}(1-0)$ & 21.4 & 22.0 & 1.022 & 0.972 & 0.562 \\
NGC 1140 & ${ }^{12} \mathrm{CO}(2-1)$ & 10.7 & 22.0 & 2.406 & 0.572 & 1.135 \\
NGC 1140 & ${ }^{12} \mathrm{CO}(3-2)$ & 18.1 & 22.0 & 1.204 & 0.818 & 0.945 \\
NGC 1140 & ${ }^{12} \mathrm{CO}(4-3)$ & 13.6 & 22.0 & 1.705 & 0.652 & 0.556 \\
NGC 1140 & ${ }^{13} \mathrm{CO}(1-0)$ & 22.4 & 22.0 & 0.983 & 1.023 & 0.053 \\
NGC 1140 & ${ }^{13} \mathrm{CO}(2-1)$ & 11.2 & 22.0 & 2.246 & 0.584 & 0.100 \\
NGC 1140 & {$[\mathrm{Cr}](1-0)$} & 12.7 & 22.0 & 1.865 & 0.626 & 0.352 \\
\hline
\end{tabular}

Notes. Assuming that the $\mathrm{CO}$ is distributed as a Gaussian with the bestfit $\theta_{\mathrm{s}}=26^{\prime \prime}$.

integrated to a circular aperture radius $\theta_{\mathrm{b}} / 2$ to obtain the aperture correction:

$$
\begin{aligned}
S_{v} & =A_{0}^{\prime} \frac{\sigma_{\mathrm{s}}^{2}}{\left(\sigma_{\mathrm{b}}^{2}+\sigma_{\mathrm{s}}^{2}\right)} \int_{0}^{2 \pi} \int_{0}^{\theta_{\mathrm{b}} / 2} \exp \left(\frac{-r^{2}}{\left(\sigma_{\mathrm{b}}^{2}+\sigma_{\mathrm{s}}^{2}\right)}\right) r \mathrm{~d} r \mathrm{~d} \phi \\
& =A_{0}^{\prime} \pi \sigma_{\mathrm{s}}^{2}\left[1-\exp \left(\frac{-\theta_{\mathrm{b}}^{2}}{4\left(\sigma_{\mathrm{b}}^{2}+\sigma_{\mathrm{s}}^{2}\right)}\right)\right] .
\end{aligned}
$$

For the case of a Gaussian source distribution, to correct the fluxes (e.g., $S_{v}$ in Jy) to a common beam size, we scaled the observed $\mathrm{CO}$ fluxes by comparing the growth-curve integrals in the different beam sizes using the analytical formula in Eq. (B.5). Figure B. 1 shows the aperture and the $T_{\mathrm{mb}}$ corrections for different transitions in the case of a Gaussian source distribution. Also shown are the exponentially-derived beam corrections for NGC 1140 (see Appendix A).

In the best-fit procedure described in the main text, we sample source FWHM $\theta_{\mathrm{s}}$ ranging from $2^{\prime \prime}$ to $40^{\prime \prime}$ with steps of $1^{\prime \prime}$, and for each of these derive a multiplicative factor $\mathrm{AP}_{\text {cor }}$ $S_{22}=S_{\text {orig }} \mathrm{AP}_{\text {cor }}$ (see Eq. (A.5)). We then apply this correction to velocity-integrated $T_{\mathrm{mb}}$ values, and consider the best fit as the one with the lowest $\chi^{2}$ value. Corrected values for NGC 1140 are given in Table B.1.

It is interesting that the Gaussian beam corrections found by the $\chi^{2}$ minimization technique are the same as the independently-derived exponential beam corrections. It seems unlikely that this is a fortuitous result, but rather that it is confirming the validity of either approach to beam corrections. 

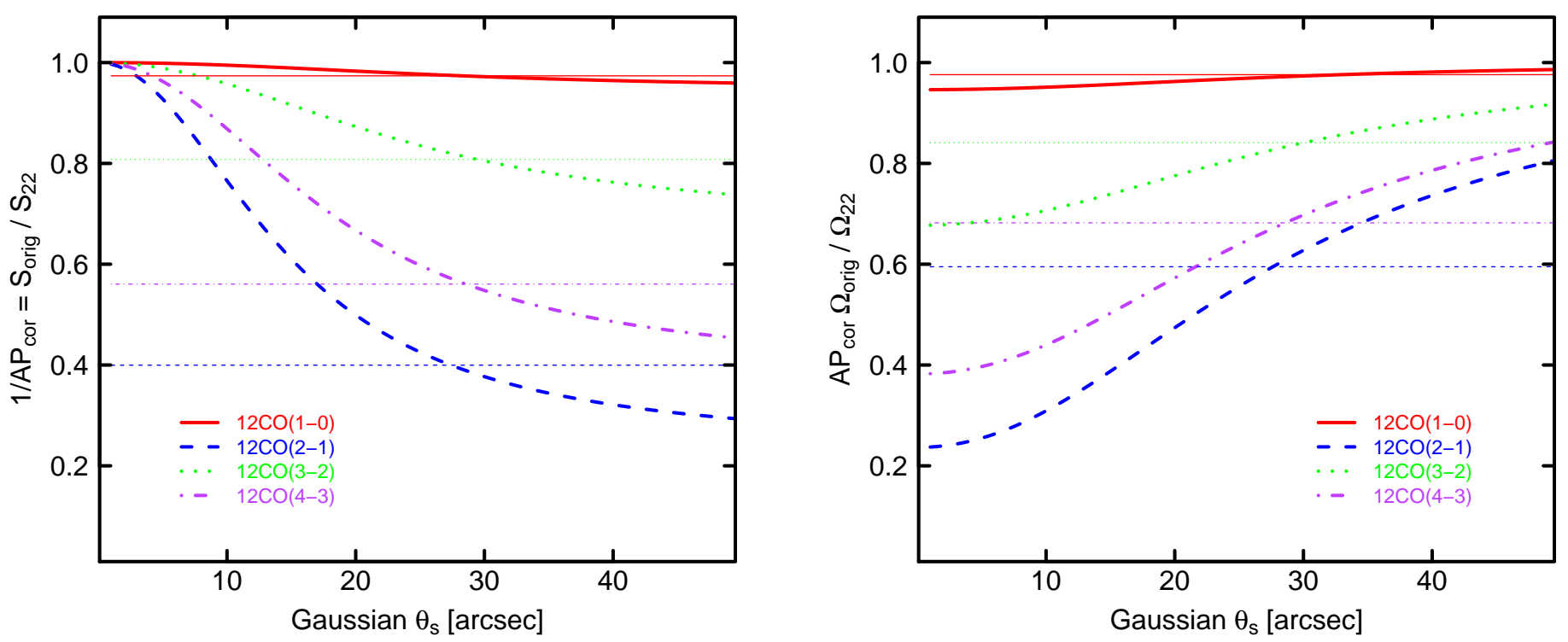

Fig. B.1. Left panel: (inverse of) aperture corrections as given in Eq. (B.5) plotted against Gaussian source FWHM $\theta_{\mathrm{s}}$. Right panel: $T_{\mathrm{mb}}$ corrections (AP corplotted against $\theta_{\mathrm{s}}$. In both panels, different ${ }^{12} \mathrm{CO}$ beams are shown by different colors as line types as described in the legend: ${ }^{12} \mathrm{CO}(1-0)$ : solid (red); ${ }^{12} \mathrm{CO}(2-1)$ : dashed (blue); ${ }^{12} \mathrm{CO}(3-2)$ : dotted (green); ${ }^{12} \mathrm{CO}(4-3)$ : dot-dashed (purple). The horizontal (light-weighted) lines of the same type correspond to the exponentially-derived quantities for aperture (left panel) and $T_{\mathrm{mb}}$ corrections (right) for NGC 1140 . They converge roughly to a common Gaussian beam size of $\theta_{\mathrm{s}} \sim 26^{\prime \prime}$ (see main text for more details). 